University of Louisville

ThinkIR: The University of Louisville's Institutional Repository

Electronic Theses and Dissertations

$12-2012$

\title{
Anxiety in older adults with dementia residing in long-term care facilities.
}

Lauren Summer Hess

University of Louisville

Follow this and additional works at: https://ir.library.louisville.edu/etd

\section{Recommended Citation}

Hess, Lauren Summer, "Anxiety in older adults with dementia residing in long-term care facilities." (2012). Electronic Theses and Dissertations. Paper 611.

https://doi.org/10.18297/etd/611

This Doctoral Dissertation is brought to you for free and open access by ThinkIR: The University of Louisville's Institutional Repository. It has been accepted for inclusion in Electronic Theses and Dissertations by an authorized administrator of ThinkIR: The University of Louisville's Institutional Repository. This title appears here courtesy of the author, who has retained all other copyrights. For more information, please contact thinkir@louisville.edu. 


\title{
ANXIETY IN OLDER ADULTS WITH DEMENTIA RESIDING IN LONG-TERM CARE FACILITIES
}

By

Lauren Summer Hess

B.A., St. Mary's College of Maryland, 2001

M.A., University of Louisville, 2008

\begin{abstract}
A Dissertation
Submitted to the Faculty of the

College of Arts and Sciences of the University of Louisville in Partial Fulfillment of the Requirements

for the Degree of
\end{abstract}

Doctor of Philosophy

Department of Psychological and Brain Sciences

University of Louisville

Louisville, Kentucky

December 2012 


\section{ANXIETY IN OLDER ADULTS WITH DEMENTIA RESIDING IN LONG-TERM CARE FACILITIES}

By

Lauren Summer Hess

B.A., St. Mary’s College of Maryland, 2001

M.A., University of Louisville, 2008

A Dissertation Approved on

August 21, 2012

by the following Dissertation Committee:

Dr. Suzanne Meeks, Ph.D.

Dissertation Director

Janet Woodruff-Borden, Ph.D. 


\section{DEDICATION}

This dissertation is dedicated to

My parents, Lawrence and Cheryl Hess, for their unfailing support, and my grandmother, Jane Hess, for her encouragement and faith in me. 


\section{ACKNOWLEDGEMENTS}

I would like to thank my mentor, Dr. Suzanne Meeks, for her support, guidance, and encouragement during my graduate career. She has provided me with a shining example of a geropsychologist in both research and clinical practice. I would like to express my gratitude to my clinical supervisors and other faculty in the Department of Psychological and Brain Sciences who have challenged me to learn and grow over the years. Thank you to my dissertation committee members for your time, patience, and support during this process. I would like to acknowledge the tireless persistence of the undergraduate research assistants who helped me collect data, especially Jordan Williams. Finally, I would like to thank my mother, Cheri. Without your guiding hand leading me to my first nursing home job, I would not be where I am today. 


\begin{abstract}
ANXIETY IN OLDER ADULTS WITH DEMENTIA RESIDING IN LONG-TERM

CARE FACILITIES

Lauren Summer Hess
\end{abstract}

August 21, 2012

Dementia, depression, and anxiety are the most common psychiatric disorders among long-term care residents and, because dementia is associated with behavioral problems that can be difficult to manage, the growing number of long-term care residents with dementia and mental health disorders presents a challenge for providing quality care. Research has established the co-occurrence of depression and anxiety and anxiety and agitation in this population. However, a consensus on the conceptualization of the co-occurrence of these constructs has not been reached. It has also been postulated that older adults with dementia may not be able to experience anxiety as conceptualized due to cognitive impairment. Many older adults with anxiety report having anxiety throughout their lives, suggesting those with dementia would continue to experience anxiety despite cognitive decline. The purpose of this study is to examine the relationships among depression, anxiety, and agitation in long-term care residents with dementia emphasizing the roles of cognitive functioning and mental health history.

Residents $(N=65)$ completed measures of mental health history, anxiety, and affect, while their nursing assistants completed measures of anxiety and agitation. Measures of depression and cognitive functioning were gathered from Minimum Data 
Sets. Confirmatory factor analyses and factorial ANOVAs were used to test the hypotheses. The results demonstrated depression, anxiety, and agitation in long-term care residents with dementia represented an overarching construct of distress. Significant relationships existed between current anxiety and agitation, as well as history of anxiety and current anxiety, irrespective of cognitive functioning.

The results of this study suggest depression and anxiety in long-term care residents with dementia may be structurally different than in younger adults and other older adult populations. Differentiating agitation from other indicators of affective distress was not possible in this sample. Instead, depression, anxiety, and agitation may all be manifestations of a global construct of distress associated with dementia. Nevertheless, history of anxiety disorder was a predictor of current anxiety. The continuity of anxiety across the lifespan, maintenance of anxiety throughout the progression of dementia, and risk for increased co-morbidity highlights the need for screening for anxiety in long-term care facilities to provide optimal care. 


\section{TABLE OF CONTENTS}

\section{Page}

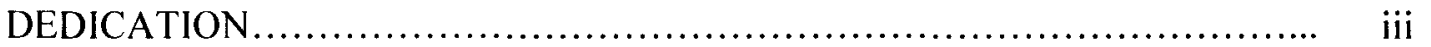

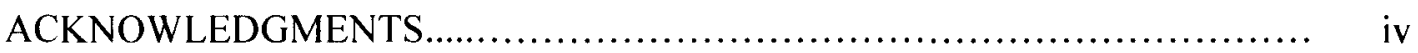

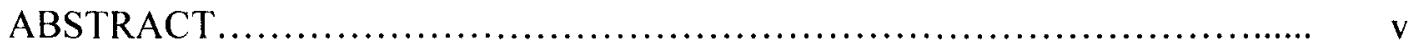

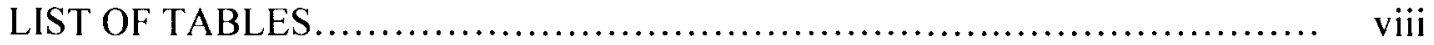

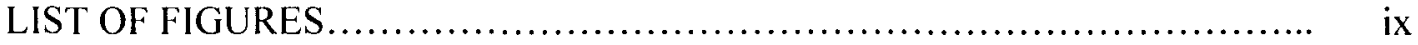

INTRODUCTION ............................................................. 1

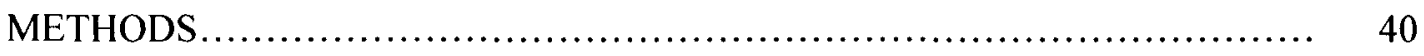

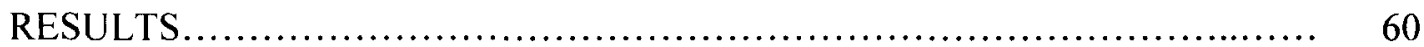

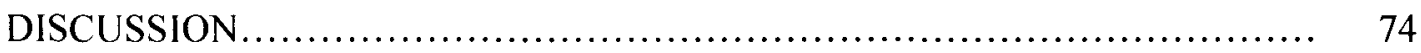

REFERENCES....................................................... 96

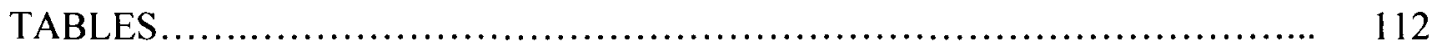

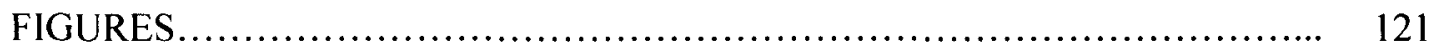

CURRICULUM VITAE .................................................... 125 


\section{LIST OF TABLES}

TABLE Page

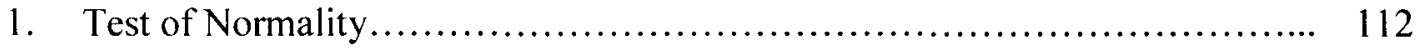

2. Demographic Characteristics of Ineligible Participants.................... 113

3. Descriptive Statistics for Ineligible Participants....................... 114

4. Demographic Characteristics of Eligible Participants..................... 115

5. Descriptive Statistics for Eligible Participants......................... 116

6. Factor Loadings for the Single Factor Model (Resident).................. 117

7. Factor Loadings for the Single Factor Model (CNA) ................... 118

8. Bivariate Correlations Measures and Their Subscales (Resident)........... 119

9. Bivariate Correlations Measures and Their Subscales (CNA) .............. 120 


\section{LIST OF FIGURES}

FIGURE $\quad$ Page

1. Initial Hierarchical Three Factor Model................................ 121

2. Initial Hierarchical Three Factor Model with Modification Indices........... 122

2. Revised Single Factor Model (Resident) ................................ 123

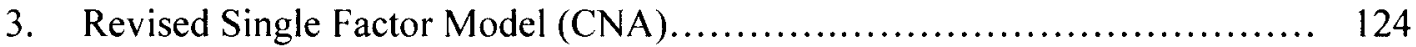




\section{CHAPTER 1}

\section{INTRODUCTION}

Older adults are the fastest growing age cohort in the United States (U.S.).

Current estimates of the population indicate that 40 million adults age 65 and over live in the U.S. (U.S. Census Bureau, 2010). By 2030 the population of adults age 65 and older is expected to almost double to 71 million people, representing $20 \%$ of the total U.S. population (He, Sengupta, Velkoff, \& DeBarros, 2005). Many older adults in the U.S. reside in long-term care facilities. According to the National Nursing Home Survey, there are approximately 16,100 long-term care facilities serving 1.5 million residents. The majority of the residents in long-term care facilities are over the age of $65(88.3 \%)$ and many are age 85 and older (45.2\%), are female (71.2\%), and are European American (85.5\%; Jones, Dwyer, Bercovitz, \& Strahan, 2009). Dementia, depression, and anxiety are the most common psychiatric disorders among older adults in long-term care (Seitz, Purandare, \& Conn, 2010). The percentage of long-term care residents who have a diagnosis of Alzheimer's disease and senile dementia at the time of admission is $8.5 \%$ and 1.4\%, respectively (Jones et al., 2009). An increasing number of older adults are being admitted to long-term care with mental health disorders (14.2\%), including anxiety and depression, and these admissions exceed the number of admissions for dementia (Fullerton, McGuire, Feng, Mor, \& Grabowski, 2009; Jones et al., 2009). Dementia, depression, and anxiety commonly co-occur and, because dementia is associated with a 
variety of behavioral and psychological problems that can be difficult to manage, the growing number of older adults with dementia and mental health disorders residing in long-term care facilities presents a challenge for providing quality care (Lenze $\&$ Wetherell, 2011).

Both depression and anxiety have been shown to be common among older adults with dementia (Ballard, Boyle, Bowler, \& Lindesay, 1996; Ferretti, McCurry, Logsdon, Gibbons, \& Teri, 2001; Mack, Patterson, \& Tariot, 1999; Neville \& Teri, 2011; Porter et al., 2003; Teri et al., 1999). Older adults with dementia most frequently report experiencing symptoms of anxiety, including worry, apprehension, fear, and irritability that may center on concerns related to cognitive impairment and the implications thereof (Ballard et al., 1996; Reisberg, Franssen, Sclan, Kluger, \& Ferris, 1989; Teri et al., 1999). Additional research has sought to identify the incidence of anxiety disorders in older adults with dementia, focusing primarily on the occurrence of Generalized Anxiety Disorder (GAD). Estimates of GAD in older adults with dementia range from 5 to $50 \%$, depending on the diagnostic criteria used (Ballard, Mohan, Patel, \& Graham, 1994; Chemerinski, Petracca, Manes, Leiguarda, \& Starkstein, 1998; Ferretti et al., 2001). Thus, research supports the experience and manifestation of anxiety symptoms and GAD in older adults with dementia. However, further research is needed to better understand the phenomenology of anxiety in older adults with dementia living in long-term care given that the presence of anxiety symptoms and GAD commonly co-occurs with symptoms of depression and mood disorders in this population.

Research has established that older adults with dementia who experience symptoms of anxiety often concurrently experience symptoms of depression and vice 
versa (Ballard et al., 1996; Ferretti et al., 2001; Teri et al., 1999). GAD and Major Depressive Disorder (MDD) also co-occur, with many older adults with dementia who meet criteria for GAD also meeting criteria for MDD, though fewer older adults with dementia with a primary diagnosis of MDD also meet criteria for GAD (Ballard et al., 1994; Ferretti et al., 2001). Much research has found that anxiety arises within the experience of depression in older adults with dementia, while other research has determined the experience of anxiety and depression are separate, but related, symptom presentations (Gibbons, Teri, Logsdon, \& McCurry, 2006; Harwood, Ownby, Barker, \& Duara, 1998; Mack et al., 1999; Mega, Cummings, Fiorelli, \& Gorbein, 1996; Tariot et al., 1995; Teri et al., 1992). The former findings suggest anxiety and depression symptoms in older adults with dementia may represent a general experience of distress rather than specific psychopathology syndromes, which has been postulated in the older adult population without dementia (Meeks, Woodruff-Borden, \& Depp, 2003); whereas the latter findings correspond to the conceptualization of anxiety and depression as being related by negative affect, but differentiated by the presence of physiological symptoms and low positive affect as has also been suggested in older adults (Cook, Orvaschel, Simco, Hersen, \& Joiner, 2004; Teachman, Siedlecki, \& Magee, 2007). A consensus on the conceptualization of the co-occurrence of anxiety and depression symptoms and disorders in older adults with dementia is lacking and additional research is needed to determine if manifestations of anxiety in older adults with dementia may actually be anxiety symptoms often experienced in depression, if anxiety and depression are separate, but related, constructs in this population, or if anxiety and depression 
experienced by older adults with dementia are best accounted for by an overarching construct of distress.

In addition to the co-occurrence of anxiety and depression, anxiety and agitation have been linked in older adults with dementia. Estimates of the incidence of agitated behaviors in older adults with dementia may be as high as $99 \%$, but more conservative estimates have found approximately $68 \%$ of older adults in this population demonstrate significant agitation (Trachtenberg, Weiner, \& Thal, 2002). Agitation in older adults with dementia has been connected to placement in long-term care and, once residing in long-term care; agitated behaviors are frequently perceived by long-term care staff as disruptive and have been associated with increased staff burden (Cohen-Mansfield, 2008; Logsdon et al., 1999; Norton, Allen, Snow, Hardin, \& Burgio, 2010; Sourial, McCusker, Cole, \& Abrahamawicz, 2008). Many of the symptoms of anxiety, including those consistent with GAD, overlap with the syndrome of agitation (e.g., restlessness) and symptoms of anxiety and agitation commonly co-occur in older adults with dementia (Logsdon et al., 1999; Mintzer \& Brawman-Mintzer, 1996; Twelftree \& Qazi, 2006). Anxiety in older adults with dementia has been correlated with increases in agitated behaviors, leading many to conceptualize agitation as the behavioral manifestation of anxiety in this population such that, as dementia progresses and cognitive functioning declines, the anxiety shifts to a predominantly behavioral presentation (Cohen-Mansfield \& Billig, 1986; Ferretti et al., 2001; Mega et al., 1996; Ownby, Harwood, Barker, \& Duara, 2000; Porter et al., 2003; Shankar \& Orrell, 2000). Increases in agitated behavior with the progression of dementia have been demonstrated repeatedly; but the findings on the correlation between anxiety and degree of cognitive impairment lack consistency, 
suggesting that equating agitation with anxiety in dementia may be inaccurate (Chemerinski et al., 1998; Cohen-Mansfield, 2008; Forsell \& Winblad, 1997; Mega et al., 1996; Orrell \& Bebbington, 1996; Porter et al., 2003; Teri et al., 1999; Starkstein et al., 2007). Moreover, agitation in older adults with dementia has been conceptualized as a separate construct characterized as behaviors driven by a variety of unmet needs and precipitated by an array of cognitive, emotional, behavioral, and environmental antecedents (Kong, 2005). Accordingly, anxiety and agitation in older adults with dementia may be separate, but related, constructs. Further examination of the relationship between them and cognitive impairment is warranted.

Many older adults with anxiety report they have had "anxiety all their lives," beginning as early as childhood, suggesting that older adults with dementia who have a history of anxiety disorders would continue to experience anxiety as their dementia progresses (p. 26; Le Roux, Gatz, \& Wetherell, 2005). Changes in cognitive functioning, though, may limit their ability to experience anxiety as currently conceptualized (Barlow, 2002). As dementia progresses, the ability to engage in higher cognitive processes diminishes, and given that the predominant models of anxiety incorporate and stress the importance of cognition (e.g., cognitive biases) in the creation and maintenance of anxiety, older adults with advanced dementia may not be able to experience anxiety as currently conceptualized. On the other hand, older adults in the earlier stages of dementia when cognitive functioning is relatively intact may be able to experience anxiety. The degree to which older adults in the earlier stages of dementia experience anxiety, and whether or not the anxiety is considered pathological, may depend on mental health history. The purpose of this study is to examine the relationship among anxiety, 
depression, and agitation in older adults with dementia residing in long-term care facilities with an emphasis on the role global cognitive functioning and mental health history play in the experience of anxiety and their ability to differentiate among anxiety, depression, and agitation in this population.

\section{Anxiety in the Older Adult Population}

Based on an extensive review of the literature, Flint (1994) reported $11 \%$ of adults age 60 and over met criteria for an anxiety disorder, and established the most prevalent anxiety disorders among older adults as being GAD (4\%) and phobias (.7-12\%), while panic disorder was rare (.1\%; Krasucki, Howard, \& Mann, 1998). More recent studies on the prevalence of anxiety among older adults report higher estimates of symptoms and disorders. For instance, Mehta et al. (2003) found an overall rate of $19 \%$ of older adults experiencing anxiety symptoms with $15 \%$ of older adults without co-occurring depressive symptoms experiencing anxiety symptoms and $43 \%$ of those with co-occurring depressive symptoms also reporting anxiety symptoms. Similarly, Cohen, Magai, Yaffee, and Walcott-Brown (2006) estimated an overall rate of anxiety symptoms to be $14 \%$. They noted, however, that older adults in their sample tended to present with subclinical anxiety (13.3\%) and estimated only $2.3 \%$ actually met diagnostic criteria for an anxiety disorder. In regard to specific anxiety disorders, Brenes and colleagues (2005) determined a GAD prevalence rate of $6.9 \%$ among adults age 60 and over; and Cohen et al. (2006) reported $8.9 \%$ of adults age 55 and over met criteria for current specific phobia.

The experience of anxiety among older adults may be lifelong and chronic, particularly in regard to GAD (Beck, Stanley, \& Zebb, 1996; Brenes, Guralnik, 
Williamson, Fried, \& Penninx, 2005). Many older adults with GAD report experiencing symptoms of the anxiety disorder "all of their lives" (p.26; Le Roux et al., 2005). Despite reports that many older adults with GAD have experienced lifetime symptoms, research has unveiled a bimodal distribution of the age of onset of GAD among older adults. The first group of older adults with GAD consists of those who had an onset during childhood or adolescence, while the second group comprises those with an onset during middle adulthood. For instance, Beck, Stanley, and Zebb (1996) found 36\% of their sample of community-dwelling older adults age 55 and older diagnosed with GAD reported an onset of GAD symptoms before the age of 15 and $39 \%$ reported an onset after the age of 39. Using a similar sample of older adults to determine the age of onset of GAD, Le Roux, Gatz, and Wetherell (2005) found 57\% of older adults had an onset of GAD before age 50 and $43 \%$ after age 50 . Important differences exist between older adults with early- and late-onset GAD. Older adults with early-onset GAD experience greater severity of GAD, more severe worry, increased co-occurrence of at least one other mental health disorder, higher rates of psychoactive medication use, and significant limitations in physical functioning (Le Roux et al., 2005). These studies demonstrate the longevity of GAD and how the enduring nature of the disorder negatively affects the lives of older adults.

The concurrence of anxiety and other mental health disorders is common among older adults (Mehta et al., 2003). Anxiety is closely linked with depression in this population. Lenze et al. (2000) reported the presence of anxiety disorders was extremely high among older adults, especially if the older adult had an existing depressive disorder; and Chou (2009) determined a high prevalence of anxiety and mood disorders in older 
adults with early- and late-onset of GAD. According to Beck and colleagues (1996), $11 \%$ of older adults diagnosed with GAD had concurrent major depression, and those diagnosed with GAD tended to report higher levels of depression. Social anxiety, panic, and GAD have been associated with increased depression in older adults (Chou, 2009; Depp, Woodruff-Borden, Meeks, Gretarsdottir, \& DeKryger, 2005; Gretarsdottir et al., 2004). Furthermore, the presence of anxiety or depression in older adults increases the likelihood of developing clinically significant symptoms of either disorder that are more severe and chronic (Brenes et al., 2005; Schoevers, Deeg, van Tilburg, \& Beekman, 2005).

\section{Moving toward research on anxiety in long-term care residents and in the} context of dementia. Research on anxiety in older adults has laid the groundwork for studies on anxiety among other older adult populations. The questions addressed in understanding anxiety in older adults have carried over to the research conducted on anxiety in older adults living in long-term care facilities and with dementia, including the occurrence of anxiety symptoms, the type of symptoms experienced, prevalence of anxiety disorders, and correlates of anxiety with the goal of estimating and assessing the presence of anxiety in the long-term care environment and in the context of dementia. As researchers have taken into account possible age-related differences in the experience and manifestation of anxiety in older adults, so, too, has the role the long-term care environment and dementia plays in the experience and expression of anxiety in older adults been considered.

\section{Anxiety in Older Adults Residing in Long-Term Care}


Knowledge of the prevalence of anxiety symptoms and disorders and their origins and correlates in older adults residing in long-term care is limited. Most research has focused on understanding anxiety in relatively healthy, cognitively intact, communitydwelling older adults (Smallbrugge, Pot, Jongenelis, Beekman, \& Eefsting, 2005). However, in a study of 333 nursing home residents age 55 and older with a Mini Mental State Examination (MMSE) score of greater than 14, Smallbrugge and colleagues (2005) found the prevalence of anxiety disorders in this population to be $5.7 \%$, subclinical anxiety disorders to be $4.2 \%$, and anxiety symptoms (at least one anxiety symptom) to be 29.7\%. Among anxiety disorders, specific phobia (3.6\%), panic disorder $(1.5 \%)$, and GAD (1.2\%) were the most prevalent, and a similar pattern was seen among those with subclinical anxiety disorders. Anxiety disorders and subclinical anxiety disorders in this population were most frequently correlated with higher MMSE score (>23) and concurrently experiencing depression (51.5\%). This study suggested the prevalence rate of anxiety disorders appears to be lower in a long-term care population as compared to community-dwelling older adults. The researchers assert the lower prevalence of anxiety disorders may be attributed to nursing home environment, which they contend offers structure and fosters a sense of safety that leads to diminished experience of anxiety. However, Smallbrugge et al.'s finding of the prevalence of anxiety disorders is similar to those from studies on community-dwelling older adults in regard to the finding of greater prevalence of subclinical anxiety. The researchers argue DSM-IV criteria may not be appropriate for a population with co-occurring mental and physical disorders, and conclude a prevalence rate of $9.9 \%$ inclusive of clinical and subclinical anxiety may be more accurate. The co-occurrence of [clinical and subclinical] anxiety and [major and 
minor] depression in this population was greater than $50 \%$. Based on this rate of cooccurrence, the researchers postulate that anxiety and depression may represent the same disorder in long-term care residents.

Follow-up analyses completed by Smallbrugge, Jongenelis and colleagues (2005) sought to explicate the occurrence and risk factors associated with anxiety, depression, and co-occurring anxiety and depression, and the dimensional quality of anxiety and depression in older adults living in long-term care facilities. The researchers argued that anxiety and depression could represent a "continuum" if the co-occurrence of anxiety and depression was dependent upon the level of severity of either anxiety or depression (p. 219). First, the researchers found the prevalence of depression in the absence of anxiety to be $17.1 \%$, anxiety in the absence of depression to be $4.8 \%$, and co-occurring anxiety and depression to be $5.1 \%$, indicating depression is more prevalent in long-term residents and that co-occurring anxiety and depression is more prevalent than anxiety in the absence of depression. Second, the researchers determined that the co-occurrence of depression and anxiety was dependent upon the severity of the disorder, such that the occurrence of major depression in anxiety increased among individuals without anxiety symptoms (3.4\%), anxiety symptoms (10.6\%), subclinical anxiety disorders (28.6\%), and clinical anxiety disorders (42.1\%). The same held true for the occurrence of an anxiety disorder in depression: no symptoms of depression (1.1\%), symptoms of depression (6.3\%), minor depression (8.5\%), and major depression (29.6\%). These findings demonstrated that the presence of either disorder may exacerbate the pathology of the other disorder, which is a finding consistent with those studies on the co-occurrence of anxiety and depression among community-dwelling older adults, and that anxiety and 
depression in older adults living in long-term care facilities existed along a continuum based on severity of disorder.

Contrary to belief that living in long-term care facilities may reduce anxiety in older adults (Smalbrugge, Pot, et al., 2005), it has also been argued that the long-term care environment may facilitate the experience of anxiety. Much of the research on anxiety in older adults living in long-term care has concerned the process of relocation into, out of, between, and within facilities. Thomasma, Yeaworth, and McCabe (1990) completed a longitudinal study on the effect of relocation on the psychological well-being of 62 independent-living and long-term care residents moving to a new facility. Using the State-Trait Anxiety Inventory-State, they found higher anxiety among long-term care residents who moved to minimal, intermediate, and skilled care units of the new facility, as compared to older adults previously residing in independent living who moved to the same type of environment. The relationship between anxiety and independent living versus long-term care was significant one week and one month after the move, indicating relocation produces lasting anxiety in individuals receiving long-term care. Moreover, the results of this study revealed that older adults residing in long-term care were far less prepared for the move as assessed by number of visits to the new facility prior to relocation and amount of information provided by the original facility regarding the moving process.

Understanding the experience of anxiety in long-term care residents. The dimensional quality of anxiety and depression in long-term care facility residents as described by Smallbrugge, Jongenelis, and colleagues (2005) is based on severity, but does not necessarily support the notion that anxiety and depression in this population 
represent a single mental health disorder. The risk factors for anxiety, depression, and co-occurring anxiety and depression examined by the researchers provide little evidence of the dimensionality of anxiety and depression. Depression in the absence of anxiety was found to be predicted by impaired vision, loneliness, and the happening of at least one negative life event in the past year; anxiety in the absence of depression was predicted by younger age and the presence of social support; and co-occurrence of anxiety and depression was predicted by pain, loneliness, and perceived inadequacy of care. Presumably, if anxiety and depression represent a unified construct, then risk factors would be relatively similar. Anxiety and depression may represent different diagnostic categories in older adults living in long-term care, and exploration of other factors predictive of anxiety and depression and co-occurrence of these disorders should be explored, such as history of mental health disorder and degree of cognitive impairment (Hudon, Voyer, Tremblay, Tardif, \& Carmichael, 2010).

In regard to the study conducted by Thomasma and colleagues (1990), greater anxiety among older adults living in and relocating to long-term care facilities may be due to the difference between the nature of independent and long-term care environments and the degree of preparation provided the residents in each group. Independent living fosters autonomy, opportunities for choice, and control over one's environment from retaining one's possessions to increased privacy and freedom of activity, whereas residing in long-term care limits autonomy, choice, and control. The results of this study revealed that older adults residing in long-term care may have been less prepared for the move as assessed by number of visits to the new facility prior to relocation and amount of information provided by the original facility regarding the moving process. The issue of 
less preparation for and predictability of moving coupled with minimal control over one's environment once relocated may be the source of increased anxiety. The researchers' finding on the relationship between lack of control and predictability and increased anxiety in older adults living in long-term care corresponds to Barlow's (2002) conceptualization of the process of anxiety in which individuals are likely to experience anxiety in situations they perceive as uncontrollable or unpredictable; though the researchers neither statistically analyzed the relationship between controllability and predictability and anxiety in long-term care residents, nor assessed premorbid anxiety in this study. Prior mental health history may also contribute to the experience of anxiety in long-term care residents, as would cognitive status, since older adults in long-term care have poorer cognitive functioning than those in more independent living environments and cognitive functioning has been related to severity of mental health disorder symptoms (Ashcraft, Owne, \& Feng, 2006; Hudon et al., 2010).

\section{Anxiety in Older Adults and Dementia}

Anxiety symptoms and disorders. The experience of anxiety symptoms among older adults with dementia is common (Ballard et al., 1996; Ferretti et al., 2001; Neville \& Teri, 2011). Research indicates older adults with dementia experience multiple symptoms of anxiety at a greater frequency and intensity than older adults without dementia (Ballard et al., 1996; Trachtenberg et al., 2002). Estimates of anxiety symptoms in older adults with dementia have ranged from 19 to $70 \%$ with the majority of symptoms experienced at the moderate to severe level (Ballard et al., 1996; Ferretti et al., 2001; Neville \& Teri, 2011; Porter et al., 2003; Teri et al., 1999). Older adults with dementia experience both the subjective feelings and physical signs of anxiety, 
specifically worry, fear, restlessness, and physiological sensations (Mack et al., 1999). To explore the types of anxiety symptoms older adults with dementia experience, Ballard, Boyle, Bowler, and Lindesay (1996) inquired about the presence and duration of several anxiety symptoms, including tension, worry, irritability, feelings of anxiety and fear, physiological symptoms, anxiety provoking situations, and panic attacks using a series of questions included in a standard assessment battery developed by the researchers in a sample of 158 older adults with dementia. The experience of tension symptoms (38.5\%) was rated most frequently, followed by subjective anxiety (worry, irritability, fear; $22 \%$ ), situational anxiety $(12.8 \%)$, physiological anxiety $(11 \%)$, and panic attacks $(1.8 \%)$. Teri and colleagues (1999) have provided additional information on the anxiety symptoms experienced by older adults with dementia. Using a 21 -item checklist designed by the researchers to assess behavioral and psychological symptoms associated with dementia, the researchers found older adults with mild to moderate dementia experienced symptoms of anxiety related to feeling anxious, fearful or apprehensive (44\%), irritable (36\%), and agitated and restless (34\%), and further implicated the subjective anxiety as an important part of the experience of anxiety in older adults with dementia.

Though symptoms are usually the focus of research on the phenomenology of anxiety in older adults with dementia, a few studies have ventured to estimate the occurrence of anxiety disorders in this population. Like the research conducted on anxiety in older adults without dementia, these studies have focused on GAD. Estimates of GAD in older adults with dementia range from 5 to $15 \%$ (Ballard et al., 1994; Chemerinski et al., 1998; Ferretti et al., 2001). The wide range of GAD diagnoses in older adults with dementia is likely due to the diverse methods used to assess anxiety in 
this population, especially the inconsistent use of diagnostic criteria by researchers. Chemerinski et al. (1998) examined the prevalence of GAD in a sample of 398 older adults with mild to moderate dementia using the Structured Clinical Interview for DSMIII-R that was completed with the older adult and a first degree relative. The researchers adhered to standard DSM-III-R criteria for GAD and determined 5\% of their sample met criteria for the anxiety disorder. Ferretti et al. (2001) established a similar rate of diagnoses of older adults with mild to moderate dementia using standard DSM-III-R criteria for GAD (5-6\%). On the other hand, Ballard et al. (1994) estimated a higher prevalence of GAD (31\%) using criteria designed for research purposes in a sample of 58 older adults with dementia. Consistent use of DSM standard criteria for GAD seems to yield comparable estimates of GAD in older adults with dementia as those in the older adult population without dementia. Deviation from standard DSM criteria may inflate estimates of anxiety disorders in older adults with dementia.

\section{Considerations for diagnosing anxiety disorders in older adults with dementia.}

The use of diagnostic criteria varies with some researchers utilizing standard DSM diagnostic criteria, while others using modified or revised criteria (i.e., eliminating the criterion that the disorder is not due to the direct physiological effects of a general medical condition). In general, those studies employing standard diagnostic criteria tend to report lower estimates of $\mathrm{GAD}$, whereas those using modified or revised criteria report higher estimates. The use of standard or revised diagnostic criteria influences the estimates of the prevalence of GAD in older adults with dementia. Although few older adults with dementia meet diagnostic criteria for an anxiety disorder, many older adults with dementia experience a variety of anxiety symptoms that warrant clinical attention 
and it has been put forth that diagnostic criteria for anxiety disorders in older adults with dementia should be developed, specifically allowing an anxiety disorder to be diagnosed in the presence of another medical condition or mental health disorder (Ballard et al., 1996; Starkstein et al., 2007). At the same time, the diagnostic criterion that the anxiety disorder is not due to the direct effects of a general medical condition or occurs during another mental health disorder may be imperative for distinguishing between having an anxiety disorder and experiencing anxiety symptoms related to distress associated with the experience of having dementia. Other factors should play a role in diagnosing an older adult with dementia with an anxiety disorder, including mental health history. A pre-existing anxiety disorder may be the best way to distinguish anxiety disorder from more general distress in this population.

Anxiety and depression. Anxiety and depression symptoms commonly co-occur in older adults with dementia (Ballard et al., 1994). In the aforementioned study conducted by Teri et al. (1999), 54\% of the older adults with dementia had multiple cooccurring symptoms of depression (social isolation, inactivity, tearfulness and crying, appears depressed) and anxiety. The commonality of anxiety and depression symptoms was replicated in a later study of 137 older adults with mild to moderate dementia and their caregivers conducted by Ferretti and colleagues (2001) who examined the relationship of anxiety and depression symptoms using the Hamilton Depression Rating Scale (HDRS), Hamilton Anxiety Rating Scale (HARS), a revised version of the depression scale from the Revised Memory and Behavior Problems Checklist (RMBPCD; appears sad or depressed, comments about hopelessness, comments about being a failure, cries, comments about loneliness, and suicidal threats), and an anxiety subscale 
derived from the same instrument (RMBPC-A: appears anxious or worried, expresses fearfulness/worry, nightmares/fear on waking, constantly restless, and fidgets/unable to sit still). Both the HDRS and RMBPC-D were modified by eliminating items overlapping with those included on the HARS and RMBPC-A. HDRS and HARS total scores were strongly associated, HDRS total scores explained $53 \%$ of the variance in HARS total scores, and when the two anxiety-related items were removed from the HDRS it explained $43 \%$ of the variance in the HARS, demonstrating symptom reports of anxiety and depression are highly related and may represent a unified construct in older adults with dementia. The variance explained in the RMBPC-A by the RMBPC-D was less than $26 \%$, which may be due differences in the symptoms assessed by the RMBPCA and RMBPC-D or that anxiety and depression in this population are separate, but related, constructs.

The close relationship between anxiety and depression symptoms in older adults with dementia has also been demonstrated in research analyzing the psychometric properties of measures designed to assess for behavioral and psychological symptoms of dementia. In a study of 555 older adults with mild to moderate dementia, Mack et al. (1999) explored the factor structure of the Behavior Rating in Dementia Scale (BRSD). The BRSD consists of eight subscales, including a depression subscale. The depression subscale contains items relating feelings ( .30$)$ and physical signs (.32) of anxiety that loaded moderately onto the subscale. Of note, the item assessing physical signs of anxiety was not originally included on the depression subscale; rather it was determined to be part of the items assessing defective self-regulation, because it was found to be conceptually inconsistent with this subscale it was ultimately assigned to the depression 
subscale (Tariot et al., 1995). Other behavioral and psychological symptoms of dementia measures have shown the relationship between symptoms of anxiety and depression. The RMBPC developed by Teri and colleagues (1992) has three subscales (depression, memory-related, and disruptive behaviors). The depression subscale includes the item "appears anxious," which has been shown to be the most frequently endorsed item on the subscale ( $M=2.3, S D=1.5 ; 0$ - never occurs to 4 - occurs daily or more) in a sample of 201 older adults with mild to moderate dementia, occurred at least once in the past week in $69 \%$ of the sample, and correlated moderately onto the depression subscale (.59; Teri et al., 1992). The results of these studies demonstrate anxiety and depression are commonly co-occurring symptoms associated with the experience of dementia; but, again, may either represent a unified construct of distress in this population or separate, but related constructs.

Other studies using measures of behavioral and psychological symptoms of dementia have provided more definitive evidence that anxiety and depression are separate, but related factors. The Behavioral Pathology in Alzheimer Disease Rating Scale (Behave-AD) consists of seven domains including those evaluating affective symptoms and anxiety and phobic disturbances. Analyses of the psychometric properties of this measure using a sample of 151 older adults with mild to severe dementia showed the affective symptoms and anxiety subscales to represent distinct, but correlated factors (Harwood et al., 1998). Similarly, factor analyses for the Neuropsychiatric Inventory (NPI) that includes an anxiety subscale showed items endorsed on the dysphoria subscale correlated moderately with those on the anxiety subscale (.40; Mega et al., 1996). Conversely, Gibbons and colleagues (2006) found the anxiety and dysphoria subscales 
did not correlate at all (.07). Given evidence that anxiety and depression subscales on measures of behavioral and psychological symptoms related to dementia are moderately or not at all correlated, it may be said that anxiety and depression in older adults with dementia are separate, but related, factors.

The co-occurrence of anxiety and mood disorders is also evident in older adults with dementia. Many older adults with dementia who meet criteria for GAD also meet criteria for MDD. Ferretti et al. (2001) reported $75 \%$ of the older adults who met criteria for GAD also met criteria for MDD. Also, Ballard et al. (1994) found $50 \%$ of older adults with dementia who met criteria for GAD also met criteria for MDD, leaving the researchers to conclude the occurrence of GAD in the absence of MDD occurred in only $15.5 \%$ of the sample.

Differentiating anxiety and depression in older adults with dementia. It appears the co-occurrence of depression may inflate estimates of anxiety diagnoses in older adults with dementia. The findings of Ferretti et al. (2001) and Ballard et al. (1994) are in line with those studies on older adults without dementia and those living in long-term care facilities. As Smallbrugge and colleagues (2005) suggested with their studies on longterm care residents, anxiety and depression in older adults with dementia living in longterm care facilities may represent a unified construct of distress. Still, evidence supports anxiety and depression may be separate, but related, constructs in this population, calling for research on other factors that may predict the existence of either disorder in this population, including a history of anxiety or depression and cognitive functioning (Hudon et al., 2010). 
Anxiety and agitation. Anxiety symptoms have been linked to the occurrence of agitated behaviors in older adults with dementia (Twelftree \& Qazi, 2006). Some of the symptoms of anxiety and agitation overlap (e.g., restlessness), which has led many to conceptualize agitation as the behavioral manifestation of anxiety that occurs as older adults with dementia become increasingly cognitively impaired (Logsdon et al., 1999; Mintzer \& Brawman-Mintzer, 1996; Shankar \& Orrell, 2000). Anxiety in older adults with dementia has been associated with increased disruptive behaviors, aggression, aberrant motor behavior, and disinhibition, all of which are included in the syndrome of agitation (Cohen-Mansfield \& Billig, 1986; Ferretti et al., 2001; Mega et al., 1996; Ownby et al., 2000; Porter et al., 2003).

To better understand the relationship between anxiety and agitation in older adults with dementia, Twelftree and Qazi (2006) assessed for the presence of symptoms of anxiety and agitated behaviors using the Cohen-Mansfield Agitation Inventory (CMAI) and the Rating Anxiety in Dementia (RAID) scale in 40 older adults with mild to moderate dementia. The researchers found a moderate correlation between CMAI and RAID total scores (.51) that was still significant (.41) after removing the overlapping item pertaining to restlessness on both scales. Further analyses revealed that the CMAI total score was significantly correlated to the apprehension and vigilance (.51) and motor tension (.46) subscales of the RAID, but did not correlate significantly with the worry (.36) and autonomic hypersensitivity (.05) subscales. Specific items on the CMAI showed significant, low to moderate correlations with the RAID total score: constant requests for attention or help (.41), complaining and whining (.34), handling things inappropriately (.33), and verbal interruptions (.32). These findings indicate a connection 
between some components of anxiety and overall agitation, as well as some verbal and behavioral aspects of agitation and anxiety generally in older adults with dementia. Still, the autonomic sensitivity subscale of the RAID was not correlated with agitation and may be used to distinguish anxiety from agitation in older adults with dementia.

The relationship between anxiety and agitation in older adults with dementia is also seen in analyses of the items assessing anxiety in instruments designed to measure agitation. Agitation measures often include items assessing anxiety, namely those relating to the older adult appearing anxious. The Agitated Behavior in Dementia (ABID) scale is a 16-item measure designed to evaluate frequency and caregiver reaction to common agitated behaviors in community-dwelling older adults and includes a single item that assesses worry, anxiety, and fear (Logsdon et al., 1999). In a study of 148 older adults with mild to moderate dementia who were selected based on a history of agitated behavior, caregiver ratings of anxiety indicated subjective feelings of anxiety occurred several times a day or more frequently. The item "worry, anxiety, and or/fearfulness" on the ABID correlated (.32) with the ABID total score, suggesting, to some extent, older adults with dementia with a history of agitated behaviors also experience anxiety as perceived by caregivers and that agitation and general anxiety are correlated concepts (Logsdon et al., 1999).

Differentiating anxiety and agitation in older adults with dementia. Irrespective of agitation as the behavioral manifestation of anxiety in older adults with severe cognitive impairment, the symptoms of anxiety and agitation co-occur in this population. There appears to be a connection between some components of anxiety and certain verbal and behavioral manifestations of agitation. The low to moderate correlations between 
anxiety and agitation in this population indicate that other aspects of anxiety and agitation differentiate them. For instance, worry and autonomic sensitivity may be used to distinguish anxiety from agitation in older adults with dementia. Given that agitation is principally a behavioral disorder, the cognitive and emotional features most likely separate anxiety from agitation, as would the history of an anxiety disorder and severity of cognitive impairment for agitation.

Anxiety and cognitive impairment. Research on the association between the presence of anxiety and cognitive impairment has revealed inconsistencies. Older adults with dementia have been found to experience more frequent and severe symptoms of anxiety as cognition becomes increasingly impaired (Teri et al., 1999; Starkstein et al., 2007). Porter et al., (2003) found a relationship between anxiety and global cognitive functioning as measured by the MMSE (mild 24-29, moderate 15-23, and severe $<15$ ) in a sample of 191 older adults with dementia. Results indicated a significant linear relationship between increased anxiety as measured by the NPI anxiety subscale and decreased global cognitive functioning $(F[1,111]=5.49, p<.05)$. Other researchers have implied anxiety symptoms tend to peak at the stage of moderate cognitive impairment, presumably when older adults with dementia are aware of their cognitive deficits and may experience anxiety related to loss of cognitive functioning (Orrell \& Bebbington, 1996). For instance, Mega et al. (1996) determined 24\% of the older adults with mild $(M \mathrm{MMSE}=23.9), 65 \%$ with moderate $(M \mathrm{MMSE}=16.1)$, and $54 \%$ with severe $(M \mathrm{MMSE}=5.7)$ cognitive impairment exhibited anxiety; however, the difference between older adults with mild, moderate, and severe cognitive impairment was not reported as significant. 
Forsell and Winblad (1997) found the mean MMSE score for older adults diagnosed with an anxiety disorder was 25.2 (range $=19-30 ; S D=3.1)$ and for older adults not diagnosed with an anxiety disorder was 25.5 (range 0-30, $S D=4.5$ ). Though the researchers used DSM-IV criteria revised to make a diagnosis of anxiety in the presence of a general medication condition, older adults with moderate and severe levels of cognitive impairment (MMSE $\leq 18$ ) were not diagnosed with an anxiety disorder in this study. Based on these data it was concluded that anxiety was not associated with cognitive impairment (Forsell \& Winblad, 1997). At the same time, Chemerinski et al. (1998) found those older adults with dementia who were diagnosed with GAD tended to have mild to moderate cognitive impairment $(M \mathrm{MMSE}=20.7, S D=5.8)$, but the relationship between GAD diagnosis and severity of cognitive impairment was not statistically significant. In the previously-referenced study conducted by Ferretti et al. (2001), the researchers found (1) combined agitation assessed by a brief four-item checklist of psychiatric symptoms as present or absent and cognitive impairment measured using the Mattis Dementia Rating Scale and the MMSE explained 17\% of the variance in HARS scores and (2) greater impairment in memory as measured by the RMBPC memory subscale in combination with depression (HDRS) and the presence of hallucinations (psychiatric symptom four-item checklist) explained a total of $26 \%$ of the variance in the RMBPC-A.

\section{Summary of the relationship between anxiety and global cognitive functioning.}

Research suggests a relationship between anxiety and cognitive impairment exists. However, the nature of the relationship is unclear. The presence of other psychological and behavioral factors may better account for this relationship, including agitation and 
depression. Additionally, mental health history may better account for the experience of anxiety in dementia. Further research is needed to determine the relationships among anxiety, depression, agitation, and cognitive impairment in older adults with dementia residing in long-term care facilities.

\section{Origins of Anxiety}

Anxiety is a biopsychosocial construct, integrating biological, physiological, emotional, cognitive, and environmental components into the etiology and process of anxiety. A genetic contribution exists for the development of anxiety and its disorders, such that this genetic contribution creates a vulnerability to distress and negative affect and predisposes an individual to respond inadequately and ineffectively in stressful situations (Barlow, 2002; Eley 2001; Mineka et al., 1998; Torgersen, 1983). Generally, an individual is likely to inherit an "anxiety proneness" that primes one to experience negative affect, distress, physiological arousal, and engage in anxious behaviors without imminent threat, danger, or harm (Barlow, 2002; Mineka et al., 1998). Via a genetic vulnerability an individual may be predisposed to experience high levels of emotionality and reactivity to stressful or anxiety-provoking situations, but environmental and psychological factors spur the development of anxiety. Temperament represents the intersection of biological and environmental vulnerabilities that may dispose an individual to develop an anxiety disorder (Clark, Watson, \& Mineka, 1994; Chorpita \& Barlow, 1998). The focus on childhood temperament and attachment is important for understanding the etiology of anxiety but also valuable to understanding the process of anxiety in adulthood, as temperament and attachment correspond to adult personality traits (e.g., neuroticism; Clark, Watson, \& Mineka, 1994; Chorpita \& Barlow, 1998; 
Mineka et al.,1998; Rapee, 2002). Clark, Watson, and Mineka (1994) have linked the temperamental characteristics of excessive emotionality and arousal to the personality traits of negative affectivity and neuroticism that are associated with the presence of anxiety in adults. However, temperamental qualities are not sufficient to facilitate the development of an anxiety disorder, and other environmental factors play a substantial role in the development of anxiety (Rapee, 2002). Early experiences in an individual's environment interact with temperament to increase the likelihood of the development of anxiety (Bögels \& Brechman-Toussaint, 2006; Chorpita \& Barlow, 1998; Rapee, 2002). Notably, early experiences with reduced control over one's environment linked to insecure attachment and overprotective parenting may foster the development of anxiety disorders in adulthood (Chorpita \& Barlow, 1998).

Implications for anxiety in older adulthood. Genetics, emotionality, physiology, and environment intersect to create and maintain anxiety disorders early in life and across the lifespan. The early origins of anxiety disorders provide support for Le Roux, Gatz, and Wetherell's (2005) finding that many older adults feel they have had "anxiety all their lives" and report "their anxiety began in childhood or adolescence" (p. 26), particularly among older adults diagnosed with GAD. Additionally, genetic predisposition to anxiety and existing personality traits (e.g., neuroticism) coupled with situations and experiences that are more likely to occur in older adulthood, such as changes in physical and cognitive status, chronic illness, and functional limitations, may maintain anxiety into older adulthood (Deer \& Calamari, 1998; Schoevers, Beekman, Deeg, Jonker, \& van Tilburg, 2003; Vink, Aartsen, \& Schoevers, 2008). Reciprocally, personality traits (i.e., neuroticism) represented as vulnerability to stress and anxiety may 
increase risk for dementia (Wilson, Begeny, Boyle, Schneider, \& Bennet, 2011). The early onset, chronicity, and longevity of anxiety into older adulthood suggest pre-existing mental health disorders may best predict anxiety in older adults with dementia (Schoevers et al., 2003). It is possible that older adults with dementia who have had "anxiety all their lives" would continue to have anxiety as their dementia progresses. However, changes in cognitive functioning may alter their ability to experience anxiety as currently conceptualized.

\section{Models of Anxiety}

Barlow's (2002) model of the process of anxious apprehension integrates emotional, cognitive, physiological, and behavioral components of anxiety and emphasizes the interactions among these components. The components of the process of anxious apprehension include negative affect, increased autonomic arousal, shift in attention and cognitive biases, impairment in behavior, and attempts at coping. First, it is important to note that Barlow differentiates fear from anxiety. Barlow defines fear as a basic emotion that occurs when one is faced with actual threat or danger and prompts one to react to the dangerous situation. Fear when faced with actual threat or danger is appropriate and the actions that ensue (fight, flight, and freeze) are important to survival. Fear in the absence of threat is considered to be a false positive or false alarm and, when paired with physiological reactions, facilitates the development of panic attacks and becomes a learned association upon which future panic attacks are based. Autonomic arousal is the basis of fear and panic. Anxiety or "anxious apprehension," on the other hand, is an affective state that incorporates the basic emotion of fear along with other negative emotions, draws on an emotional memory network, and is future-oriented. 
Anxious apprehension is characterized by general distress and negative affect that is connected to cognitive processes that interact within a biological system (Barlow, 2002; Chorpita \& Barlow, 1998; Mineka et al., 1998). The negative affect experienced in anxiety is exemplified by a sense of uncontrollability of a potentially threatening or dangerous event or when one is uncertain about one's ability to control important outcomes in one's environment; and, as such, anxiety may be described as a certain degree of helplessness in the ability to determine whether or not a negative outcome will occur (Barlow, 2002; Chorpita \& Barlow, 1998; Mineka et al., 1998). The negative affect one experiences when confronted with what one perceives to be an uncontrollable event, situation, context, etc. creates a shift in attention and an increased concentration on internal processes, including self-evaluation of one's ability to respond to the experience, and anxiety-related physiological arousal. The shift of attention to an internal focus further increases the salience of the negative emotion and intensifies physiological arousal, and this intensification leads to narrowing of attention to possibly threatening or dangerous cues and cognitive biases in attention and interpretation (Barlow, 2002; Mineka et al., 1998). The focus on and scanning for potentially threatening or dangerous cues in the environment (hypervigilance) impairs one's ability to concentrate and perform, confirming negative self-evaluations regarding one's ability to respond and the belief that one is unable to control personally important outcomes (Barlow, 2002). When anxious apprehension becomes chronic, one is likely to employ in maladaptive attempts to cope; including avoidance of experiencing anxious apprehension by avoiding specific events, situations, contexts, etc. and engaging in worry. However, these maladaptive attempts to cope maintain anxious apprehension. 
Barlow's theory of anxiety in the context of dementia. In accordance with Barlow's (2002) conceptualization of anxiety, older adults with dementia may be able to develop and experience anxiety. First, it has been demonstrated that adults experience breadth and depth of emotions consistent throughout older adulthood and that older adults with dementia express both positive and negative affect (Carstensen \& Turk-Charles, 1994; Labouvie-Vief, 2008; Lawton, Van Haitsma, \& Klapper, 1996); therefore, older adults with dementia are capable of experiencing the negative affect associated with helplessness and uncontrollability that is at the heart of Barlow's model. Moreover, as dementia progresses, the environment with which older adults with dementia interact becomes increasingly confusing. The nature of their environment and the people in it may create and maintain the feelings of helplessness and uncontrollability associated with anxiety, especially if the older adult with dementia resides in long-term care - an environment that may foster feelings of uncontrollability and unpredictability (Thomasma et al., 1990). According to Barlow, anxiety is a "unique, coherent cognitiveaffective structure;" suggesting that, though the negative affect may be primary, it is the relationship between affect and cognition that comprises the experience of anxiety ( $\mathrm{p}$. 64). The negative affect leads to shifts in attention toward internal processes and a selfevaluative focus that further intensifies the negative affect, but also leads to biases in attention, interpretation, and memory. Here the ability to experience anxiety in dementia becomes questionable. As dementia progresses, the disease destroys parts of the brain that control memory, language, reasoning, sensory processing, and conscious thought; suggesting older adults with advanced dementia may not be capable of the higher cognitive processes associated with anxious apprehension. The cognitive biases seen in 
anxious apprehension are confounded by the nature of dementia. The ability to accurately attend to, interpret, and remember one's experience and environment is affected by the experience of anxious apprehension and the progression of dementia. Given that advanced dementia creates profound changes in cognitive processes, one may venture that older adults with advanced dementia are not experiencing anxious apprehension per se, but rather fear related to an inability to fully comprehend what they believe to be a threatening or dangerous situation. From the perspective of the older adult with advanced dementia, the threat is very real, which evokes the corresponding emotion of fear and the appropriate reaction of fight, flight, or freeze. On the other hand, older adults in earlier stages of dementia wherein higher cognitive processes are declining, but are still relatively intact, may be able to experience anxious apprehension according to Barlow's conceptualization. Whether or not an older adult in earlier stages of dementia experiences anxious apprehension in a given context, and whether or not the anxious apprehension experienced may be considered pathological, most likely will be determined by his or her mental health history since anxiety and its disorders frequently endure from earlier in life.

\section{Theories of Anxiety in Older Adults with Dementia}

It has been suggested that older adults in later stages of dementia may not be able to experience anxiety because of a lack of insight into their dementia and cognitive impairment (Cohen, 1998). Additionally, the issue of anxiety in older adults with dementia is complicated by the fact that many older adults lose the ability to communicate verbally. Cohen (1998) suggests that researchers and clinicians often confuse the inability to communicate verbally with the inability to experience emotions 
when, in fact, older adults may continue to experience emotion in connection to experiences regarding the progression of their dementia. Rather, Cohen points out that certain triggers have been established as provocative in anxiety disorders (i.e., objects in specific phobia, people in agoraphobia, performance in social phobia, etc.) may be applied to the reality of older adults with dementia.

Cohen (1998) argues that older adults with dementia may be able to experience anxiety despite limitations related to cognitive decline, and describes four types that they may experience and compares them to those anxiety disorders described in the DSM. First, older adults with dementia may experience anxiety when placed in situations that exceed their abilities and create performance demands. The author refers to this type of anxiety as "challenge anxiety" and likens it to social phobia in which performance, even on something as seemingly simple as answering a question, leads to "extreme anxiety like manifestation" that may be of such magnitude that it could result in a "catastrophic reaction" (p. 106). Cohen describes the catastrophic reaction as having both cognitive (e.g., frustration) and behavioral (e.g., restlessness) and somatic (e.g., sweating) components, and notes that this tendency to catastrophic reactions - be they cognitive or behavioral - are typical reactions often seen in anxiety disorders. Second, older adults with dementia may experience anxiety when confronted with unfamiliar objects, situations, or settings (e.g., different environments, unfamiliar surroundings, different caregivers). This type of anxiety is "unfamiliarity anxiety" and is similar to specific phobias and agoraphobia. Again, older adults with dementia may experience catastrophic reactions manifested as wandering, vocal outbursts, and the like when challenged by the unfamiliar and they may subside when older adults return to familiar surroundings. 
Third, older adults may experience anxiety when they are not engaged by their surroundings. The author compares this to the experience of separation anxiety in children and refers to this as the "isolation" of dementia, which produces distress and negative affect (p. 107). Fourth, adults with dementia may experience anxiety when their surrounding environment is unstructured. Cohen suggests that people with dementia may experience a sense of unease when structure is lacking in their environments, specifically in social situations; which, again, may be manifested as catastrophic reactions that may be misconstrued as problematic behavior by others.

Cohen (1998) takes on the issue of limited cognitive capacity in older adults with dementia and the assertion that they cannot experience anxiety because of cognitive impairment. The author puts forth the belief that young children who have underdeveloped brains can still experience separation anxiety; and, therefore, older adults with deteriorating brains can still experience the same feelings associated with separation anxiety in children. Cohen believes that the systems are still in place for interpreting/misinterpreting that can lead to frustration and catastrophic reactions in older adults with dementia. Cohen's conceptualization of anxiety in older adults with dementia focuses on the experience of negative affect associated with living in a confusing world. The negative affect experienced by older adults with dementia that Cohen stresses as an indication of anxiety in this population may be indicative of general distress. Moreover, this conceptualization of anxiety in older adults with dementia considers separation anxiety as resulting from unmet needs, which is one of the prominent theories of the development and maintenance of agitated behaviors. 
James (1999) has proposed another model to explain the experience of anxiety in older adults with dementia based on Beck's (Beck, Emery, \& Greenberg, 1985) cognitive model of anxiety. James' model of anxiety in older adults with dementia relates the manifestation of anxiety to their current cognitive status, highlighting that changes in cognitive status associated with dementia, and consequent changes in information processing, will create "abnormal interpretations" or cognitions related to oneself, the world, and the future (p. 346). James focuses on the relationship between these cognitive distortions and the coping strategies used by older adults with dementia that are often misinterpreted by others as "dysfunctional behaviors and actions" (p. 347). More specifically, James hypothesizes older adults with dementia may have anxious cognitions about themselves, such as "I am afraid but I'm not always sure why," that reflect vulnerability, which Beck emphasizes as the core of anxiety disorders. They may also have anxious cognitions about the future, such as "Things just seem to be getting worse," that indicate believing the world is unpredictable and about the world, such as "I don't understand what's going on," that echo the belief that the world is a scary place (p. 348). These cognitions then drive "challenging behaviors," including pacing, wandering, withdrawal, avoidance, and aggression (p. 348). As with Cohen's (1998) model, James' (1999) cognitive model of anxiety in older adults with dementia falls short of addressing the substantial overlap between the "challenging behaviors" resulting from anxious cognitions and need-driven agitated behaviors.

\section{Theories of Agitation in Older Adults with Dementia}

Definition of agitation. Cohen-Mansfield defines agitation "as inappropriate verbal, vocal, or motor activity that is not explained by needs or confusion per se [that is] 
always inappropriate" as characterized by the observer of the behavior according to three guidelines: (1) it is abusive or aggressive toward self or others, (2) the behavior may be performed at an inappropriate frequency, and (3) the behavior is not appropriate for the context in which it occurs (p.712, Cohen-Mansfield \& Billig, 1986). Moreover, the agitated behavior results from a need that is unapparent or the expression of an unmet need that is not clear and is distinguished from agitated behaviors that may result from a condition other than dementia. According to Cohen-Mansfield, agitated behaviors are largely aimless, purposeless, and do not result directly from an unmet need. Additionally, Cohen-Mansfield and Billig (1986) suggested that the behaviors may be organized according to physical or verbal and aggressive and non-aggressive, resulting in four categories of behavior: physically aggressive, physically non-aggressive, verbally aggressive, and verbally non-aggressive. Cohen-Mansfield's definition of agitated behavior is the most quoted definition of agitation in the literature and is frequently cited as the most comprehensive definition of the syndrome of agitation.

Cohen-Mansfield (1986) coupled the publication of the conceptualization of agitation with an exploratory study of agitated behaviors in older adults residing in dementia care units of long-term care facilities with the express goal of demonstrating the need for an objective means for assessing agitation. A comprehensive list of agitated behaviors that included pacing or aimless wandering, inappropriate dressing or disrobing, spitting, cursing or verbal aggression, constant unwarranted requests for attention or help, or repetitive questions or sentences, hitting or kicking, throwing things, making strange noises or screaming, biting, trying to get to a different place, complaining or being negative, hurting self or others, and general restlessness was developed. Data analyses 
centered on the intercorrelation among the behaviors, comparisons of agitated and nonagitated older adults, and attributions of reasons for the behaviors.

The results of this study showed that the most frequently exhibited agitated behaviors were constant requests for attention or help, repetitive questions or sentences, general restlessness, cursing and verbal aggression, pacing and wandering, and complaining and negativism. These behaviors were exhibited several times a day to several times an hour. Many of the behaviors were highly correlated with each other. Exploratory factor analyses revealed three separate, but related, factors: (1) physically aggressive behavior - biting, spitting, hitting, screaming, and throwing objects; (2) verbally aggressive behavior - cursing and verbal aggression; and (3) non-aggressive behavior - pacing, dressing or disrobing inappropriately, and constant requests for attention. The items loaded moderately $($ screaming $=.39)$ to highly $($ cursing $=.93)$ onto their respective factors. To some extent the exploratory factor analysis supports CohenMansfield's conceptualization of agitated behaviors as being separated along physical and verbal and aggressive and non-aggressive lines. However, four distinct factors (physically aggressive and non-aggressive and verbally aggressive and non-aggressive) were not found. Agitated older adults with dementia tended to exhibit more behaviors than non-agitated older adults with dementia, though the statistical significance of this difference was not explored or reported (Cohen-Mansfield, 1986). Based on this general trend, Cohen-Mansfield (1986) distinguished agitated older adults with dementia from non-agitated older adults as having exhibited at least one behavior at least several times per day in the past week and a second behavior once or twice in a week-long period; whereas non-agitated older adults were classified as such by never exhibiting agitated 
behaviors or by demonstrating agitation less than once a week. Cohen-Mansfield (1986) also explored staff attributions of the origins of agitated behaviors. Many of the staff attributed the manifestation of agitated behavior to frustration due to loss of control during activities of daily living, invasion of personal space, the behavior of other residents, confusion due to lack of structure, loneliness and need for attention, depression, and past problems among others. Cohen-Mansfield grouped the possible reasons for agitation into mood and needs, events, disabilities, and past issues. This study demonstrated that older adults with dementia exhibit a variety of agitated behaviors, agitated and non-agitated behaviors may be distinguished based on the frequency of the behavior, and agitated behaviors may arise for a variety of reasons. Still, CohenMansfield asserted agitated behaviors do not result from unmet needs.

Criticism of Cohen-Mansfield's definition of agitation. Cohen-Mansfield's work (Cohen-Mansfield, 1986; Cohen-Mansfield \& Billig, 1986) forms the foundation for subsequent studies on the frequency and types of agitated behaviors older adults with dementia exhibit and the variables associated with the manifestation of these behaviors. There have been several criticisms of the Cohen-Mansfield definition of agitation, including that the variety of behaviors it encompasses is too broad, the inappropriateness of the behavior is subjective and determined by an individual other than the older adult, and that distinguishing between need-driven and non-need-driven behavior is difficult (Kong, 2005).

Agitation as a consequent of unmet needs. Algase and colleagues' (1996) conceptualization of agitation in older adults with dementia differs markedly from Cohen-Mansfield's in that these authors believe that agitated behavior results from the 
unmet needs of older adults with dementia. Their conceptualization of agitation is based on the older adult's purpose and meaning when displaying behaviors like wandering, vocalizations, and aggression. Thus, need-driven, dementia-compromised behaviors are expressions of the older adult's goals or needs rather than problematic and disruptive behaviors that occur in the context of predisposing (i.e., neurological, cognitive, health, and psychosocial) and precipitating factors (i.e., interpersonal interactions, physical environment, and social environment). These behaviors are the most effective and efficient means to communicate needs and goals given impairment in cognitive function, neurological function, perceptual and language skills, and functional ability. For instance, wandering may arise from an unmet need to be physically active, vocalization (e.g., shouting or screaming) may result from discomfort during the performance of activities of daily living, and aggression may surface when an older adult is frustrated that they cannot meet their own needs. Algase et al.'s conceptualization of need-based agitated behavior emphasizes understanding agitation from the perspective of the older adult with dementia, rather than labeling it as problematic or disruptive, and stresses the importance of understanding the older adult's history and surroundings when interacting with and managing these behaviors.

Integration of theories of agitation. In a review of the extant literature Kong (2005) attempted to clarify and integrate current conceptualizations of agitation. Kong notes that there appears to be a consensus that agitation is observed motor or verbal/vocal activity that is excessive (frequency, duration, and intensity), inappropriate (as judged by an observer to the setting, situation, and interaction), repetitive (behaviors, mannerisms, statements, and vocalizations), non-specific (broad and multi-dimensional), and 
observable (usually based on observations or recall from informants). Kong contends that, though agitation is largely behavioral and must be because of impairments in the ability to communicate among older adults with dementia, agitation has cognitive and emotional components that are mainly attributed to shifts in mood, worry, fear, and misinterpretation of the environment and may be considered expression of feelings or need rather than problem behaviors. Agitation may be precipitated by a number of factors including patient-related, environmental, and interpersonal that may directly cause agitation or may lead to intermediary factors (i.e., discomfort, unmet needs, misinterpretation) that cause the physical, verbal, aggressive, and non-aggressive behaviors associated with agitation. Of note is Kong's assertion that agitation may arise from changes in mood, worry, and fear, indicating an overlap of the construct with those of anxiety and depression and suggesting behavioral problems associated with dementia frequently arise from emotional responses related to cognitive impairment and changing environmental circumstances.

\section{Hypotheses}

Hypothesis 1. The experience of anxiety symptoms and disorders among older adults, older adults living in long-term care, and older adults with dementia has been well-established. Additionally, anxiety and depression symptoms and disorders have been determined as commonly co-occurring in these populations. The co-occurrence of anxiety and agitation in older adults with dementia has also been established in community-dwelling and long-term care environments. However, whether or not the anxiety, depression, and agitation represent separate, but related constructs, or are 
included within a general construct of distress, in older adults with dementia residing in long-term care facilities has yet to be determined.

Anxiety, depression, and agitation in older adults with dementia living in longterm care facilities will represent separate, but related, constructs in this population. Anxiety and depression will be unified by negative affect, and anxiety and agitation will be unified by shared behaviors.

Hypothesis 2. Although extensive research has been conducted on the prevalence of anxiety symptoms and disorders in older adults with dementia, little research has been conducted on the predictors of the experience of anxiety symptoms in this population and the presence of an anxiety disorder. Some research has been conducted on the relationship between degree of cognitive impairment and severity of anxiety symptoms in older adults with dementia, but the findings are inconsistent. At the same time the relationship between anxiety and agitation, such that as dementia progresses agitation is hypothesized to be the behavioral manifestation of anxiety.

Evidence of anxiety as measured by the RAID will be predictive of agitation as measured by the CMAI in older adults with dementia. This relationship will be moderated by global cognitive functioning as measured by the Brief Interview for Mental Health Status.

Hypothesis 3. Research and theories on the etiology of anxiety demonstrate anxiety is a lifelong process and that many anxiety disorders noted in older adulthood continue from younger adulthood. Though the prevalence of anxiety symptoms and disorders has been researched, little research has been conducted on the relationship between mental health history and the presence of anxiety symptoms and disorders in 
older adults with dementia. In fact, many research studies on anxiety in older adults with dementia have excluded participants based on a history of mental health disorder. Preexisting mental health disorders have been linked to the presence of anxiety in dementia, but have been rarely studied, signifying a direction for future research (Chemerinski et al., 1998; Forsell \& Winblad, 1997; Ownby et al., 2000).

Evidence of pre-existing mental health disorder will be predictive of current anxiety in older adults with dementia residing in long-term care facilities as measured by the RAID. The relationship between mental health history and current anxiety will be moderated by global cognitive impairment as measured by the Brief Interview for Mental Health Status. 
CHAPTER 2

METHODS

\section{Sample}

In order to participate in the study, nursing home residents needed to meet the following criteria: (1) Currently residing in a long-term care facility; (2) at least 65 years of age or older; (3) able to understand and respond in English and communicate orally with the investigators; (4) have a diagnosis of dementia as indicated in their medical charts; (5) not currently participating in a research study on anxiety, agitation, depression, or dementia; and (6) able to provide informed consent or assent.

Because the goal of the study was to assess anxiety in long-term care residents with a range of cognitive impairment related to dementia, residents were not excluded from the study based on cognitive status. Often, older adults with more advanced stages of dementia are excluded from research studies due to an inability to provide informed consent. When it was determined that the resident was not able to provide informed consent, consent was obtained via proxy and written assent was attained from the resident.

Participants were not excluded based on a history of a mental health disorder. Very few studies have examined the relationship between pre-existing mental health disorders (e.g., anxiety or mood disorders) and the presence of anxiety in dementia, and many studies have excluded participants based on the presence of a mental health disorder. Pre-existing mental health disorders have been linked to the presence of anxiety 
in dementia and, therefore, individuals were not excluded from the study based on a prior psychiatric history.

\section{Measures}

Minimum Data Set (MDS). The MDS is a standardized, federally-mandated, assessment instrument administered at admission, updated quarterly and re-administered annually in all nursing homes. It is completed by nursing home staff. The MDS contains information about medical diagnoses, medical treatments, therapies, activities of daily living functioning, cognitive functioning, recreational preferences, and mood (Saliba \& Buchanan, 2008). The MDS supplied demographic data, including the resident's age, sex, race/ethnicity, marital status, pay type, education, length of time in the facility, and prior living arrangement. In addition to the demographic data collected, information on residents' behavior, functional status, active disease diagnoses, medications, and special treatments and procedures was collected via the MDS.

A new version of the MDS, the MDS 3.0, was implemented in nursing homes nationwide in October 2010. This version of the MDS has demonstrated improved reliability and validity over the MDS 2.0, and now includes measures for cognitive status and mood completed by interview with the resident. In the event that the resident was unable to respond to the measures, the assessment may be conducted with the resident's primary staff caregiver. The measures of cognitive status and mood have improved efficiency and clinical usefulness over the measures included in the previous version of the MDS, and have been noted by nursing staff to provide further insight into residents' cognitive status and emotional well-being (Saliba \& Buchanan, 2008). 
The Brief Interview for Mental Health Status (BIMS). The BIMS is a sevenitem measure of cognitive status included in the MDS 3.0. The BIMS assesses cognitive status via repetition of three words, temporal orientation, and recall. Scores range from 0 to 15 with higher scores indicating better cognitive status. The BIMS has been used to classify residents' cognitive status as intact/borderline (13-15), moderate impairment (812), and severe impairment $(<8)$. As indicated in the final report on the development and validity of the MDS 3.0, the inter-rater reliability of the BIMS was excellent between trained assessors $(\kappa=.98)$ and between trained assessors and facility nurses $(\kappa=.97)$. The BIMS was validated against the Modified Mini-Mental Status Exam, demonstrating a high correlation between the two measures $(r=.73, p<.01)$. The sensitivity and specificity of different BIMS cut scores for detecting any cognitive impairment and severe cognitive impairment was also examined. For any cognitive impairment, the optimal cut score is 12 , yielding a sensitivity of $69.7 \%$ and specificity of $85.6 \%$; and for severe cognitive impairment, the optimal cut score is 10 with sensitivity of $94.4 \%$ and specificity of $78.6 \%$ (Chodosh et al., 2008; Saliba \& Buchanan, 2008).

Patient Health Questionnaire-9 (PHQ-9). Also included in the MDS 3.0, the PHQ-9 is a nine-item measure that assesses symptoms of depression experienced by the resident during the past two weeks. Items are rated according to symptom presence (Yes, No, or No Response), and symptom frequency (1 Day/"Rarely"-0 to 12-14 Days/"Nearly every day"-4). Scores range from 0 to 27 , and may be used to classify residents' depression as none (0-4), mild (5-9), moderate (10-14), moderately severe (15-19), and severe (20-27). The inter-rater reliability of the PHQ-9 as noted in the final report on the development and validity of the MDS 3.0 was excellent between trained assessors ( $\kappa=$ 
$.94)$ and between trained assessors and facility nurses $(\kappa=.96)$. The PHQ-9 was validated against the Modified Schedule for Affective Disorders and Schizophrenia $(\kappa=$ .69) and the Cornell Scale for Depression in Dementia $(r=.63, p<.01)$, demonstrating moderate correlation between the PHQ-9 and other measures of mood disorders typically used in nursing homes (Saliba \& Buchanan, 2008).

Mental health history. To examine the relationship between history of a mental health problems and current experience of anxiety in older adults with dementia, history of mental illness and management was evaluated using four self-report items developed by Vahia et al. (2010) and screening questions for mood and anxiety disorders derived from the Structured Clinical Interview for DSM-IV-TR for Axis I Disorders (SCID; First, Gibbon, Spitzer, \& Williams, 2001). To ascertain the mental health history and management of mental health problems of older adult women participating in a longitudinal study on subclinical depression and perceptions of successful aging, Vahia and colleagues asked participants to respond to four self-report yes/no questions: (1) "Have you ever been diagnosed with a mental or emotional problem?" (2) "Have you ever been in treatment with a mental health professional?" (3) "Have you ever been prescribed medication for a mental or emotional problem?" and (4) "Have you ever been hospitalized for such a problem?" (p. 215). The researchers found participants with clinically significant depression as measured by the Center for Epidemiological Studies Depression scale had higher percentages of positive history for all self-report questions, and participants with subclinical depression had higher percentages of a history of diagnosis and medication prescription than individuals who had not been diagnosed with 
depression. These results suggest the self-report questions have some validity in screening for history of mental illness, in general.

Specific questions regarding residents' history of depression and anxiety were used from the SCID. The SCID includes an optional 12 question module that screens for substance abuse, anxiety disorders, and eating disorders. The questions relating to anxiety disorders screen for panic disorder, agoraphobia with history of panic disorder, social phobia, specific phobia, obsessive compulsive disorder, obsessions/compulsions, and GAD. The anxiety disorder screening questions were taken from the introductory question to each anxiety disorder sub-module of the anxiety disorders module of the SCID. Although questions regarding mood disorders are not included in the screening module, portions of the SCID may be selected for relevancy to researchers' particular interests (First et al., 2001). Therefore, the introductory questions to each of the past mood disorder sub-modules were used to screen for depression and mania.

The reliability of the SCID when used with older adults has been examined using the SCID based on the DSM-III-R. Segal and colleagues (1995) found the inter-rater reliability for mood disorders to be .79 and anxiety disorders to be .73 . More specifically, the inter-rater reliability for major depression was .90 , dysthymia was .53 , and panic disorder was .80 . The SCID has recently been used to determine the prevalence of current and lifetime anxiety disorders in older adults, though the reliability and validity of the SCID has not been established with this population for specific anxiety disorders (Flint, Peasely-Miklus, Meyers, Rothschild, \& Whyte, 2010; Gum, King-Kallimanis, \& Kohn, 2009). Inter-rater reliability of determining the presence or absence of any current anxiety disorder using the SCID with an older adult population has been reported as 
excellent ( $\kappa=1.0$; Sagen et al., 2009). Research on the reliability and validity of the SCID screening questions is very limited. However, a study conducted by Dalrymple and Zimmerman (2008) to examine the performance of the SCID screening questions when used with psychiatric outpatients found the screening question for lifetime social phobia ("Is there anything that you have been afraid to do or felt uncomfortable doing in front of other people, like speaking, eating or writing?") yielded a sensitivity of $92 \%$ and specificity of $69 \%$. Given the purpose of these questions, the sensitivity and specificity of appeared adequate in determining mental health history.

Philadelphia Geriatric Center Affect Rating Scale (ARS). The ARS is a 10item measure designed to quickly assess state and trait positive (i.e., energetic, interested, warmhearted, happy, and content) and negative (i.e., depressed, sad, worried, annoyed, and irritated) affect with a diverse population of older adults - community-dwelling to residential, healthy to frail (Lawton, Kleban, Dean, Rajagopal, \& Parmalee, 1992). The ARS was developed to be clinician administered whereby individuals rate each item on a scale from 1 (not at all) to 5 (very strongly) in response to the prompt "How are you feeling right now? Are you..." (p. 231). Total scores for positive and negative affect are computed by adding the numerical ratings for the five items composing their respective subscales. Thus, scores for positive or negative affect range from 5 to 25 with higher scores indicating higher positive or negative affect.

ARS has demonstrated strong psychometric properties and a stable factor structure across adulthood (Lawton et al., 1992; Lawton, Van Haitsma, \& Klapper, 1996). As part of a larger study to examine the factor structure of the ARS, Lawton and colleagues (1992) determined a two-factor solution consisting of positive and negative 
affect provided the best fit for a sample of older adults living in long-term care $(N=486$; $M$ age $=83.5, S D=6.06 ; 30.7 \%$ male), such that $\mathrm{GFI}=.96$ and $\chi^{2}(33)=111.1, p<.05$. The correlation between the factors was -.21 . Concurrent validity was tested using the Geriatric Depression Scale (GDS). As expected, it was found the positive affect index was negatively correlated with the GDS $(r[486]=-.68, p<.05)$, whereas the negative affect index was positively correlated $(r[486]=.61, p<.05)$.

As a follow-up to the aforementioned study, Lawton, Van Haitsma, and Klapper (1996) examined directly observed affect in a population of older adults with dementia residing in a nursing home $(N=97)$ using a six-item version (pleasure, interest, contentment, sadness, worry/anxiety, and anger). The researchers found that interest (87\%) and contentment (68\%) were displayed most often displayed, whereas anger (5\%) was displayed the least. Inter-rater reliability for each of the items was very good, ranging from .76 to .89 (Lawton et al., 1996).

Rating of Anxiety in Dementia (RAID). The RAID is an 18-item scale designed to assess four components of anxiety in older adults with dementia: (1) worry (i.e., worry about physical health; worry about cognitive performance; worry about finances, family problems; worry associated with false beliefs and/or perceptions; and worry over trifles), (2) apprehension and vigilance (i.e., frightened and anxious, sensitivity to noise, sleep disturbance, irritability), (3) motor tension (i.e., trembling, motor tension, restlessness, and fatigueability and tiredness), and (4) autonomic hypersensitivity (i.e., palpitations; dry mouth; hyperventilating and shortness of breath; dizziness or light-headedness; and sweating, flushes or chills, and tingling or numbness of fingers or toes; Shankar, Walker, Frost, \& Orrell, 1999). The symptoms of anxiety are rated on a three-point scale (0 - 
absent to 3 - severe), and ratings are based on symptoms occurring during the previous two weeks. Scores range from 0 to 54 , with higher scores on the instrument suggesting greater anxiety severity.

Shankar, Walker, Frost, and Orrell (1999) assessed the psychometric properties of the RAID. From a sample of 83 community-dwelling older adults with Alzheimer's disease, vascular and other dementias, the scale demonstrated sufficient inter-rater (item agreement ranged from .51 to 1 ) and test-retest reliability (item test-retest reliability ranged from .53 to 1$)$, as well as high total scale $(\alpha=.83)$ and moderate to high subscale (worry, $\alpha=.65$; apprehension and vigilance, $\alpha=.67$; motor tension, $\alpha=.51$; and autonomic hypersensitivity, $\alpha=.74$ ) internal consistency. Analyses for concurrent validity were conducted comparing that RAID with the Clinical Anxiety Scale (CAS), Anxiety Status Inventory (ASI), and Cornell Scale for Depression in Dementia (CSDD). Because several of the items on the RAID and the CSDD overlap, all similar items were eliminated before conducting analyses. The results of these analyses revealed that the RAID correlated moderately with the CAS $(r[83]=.54, p<.01)$, ASI $(r[83]=.62, p<$ $.01)$, and the CSDD $(r[83]=.69, p<.01)$. A modified version of the DSM-IV criteria for GAD that allowed the diagnosis of GAD in the presence of a medical disorder was used to examine criterion validity. Those who met revised criteria for GAD had significantly higher RAID scores than those who did not. Non-parametric tests revealed the RAID was able to distinguish between older adults with clinically significant levels of anxiety by using the cut score of 11 to differentiate between clinically significant and subclinical anxiety with a sensitivity of $90 \%$ and specificity of $78.5 \%$. To assess the 
construct validity of the RAID a factor analysis was conducted that revealed five factors that accounted for $63 \%$ of the variance (Shankar et al., 1999).

More recent research on the psychometric properties of the RAID revealed satisfactory internal consistency, reliability, inter-rater reliability, and construct validity (Snow et al., 2012). A sample of 32 community-dwelling older adults with dementia and corresponding informants completed measures of anxiety, depression, relationship quality, and quality of life along with the RAID. Analyses demonstrated good internal consistency reliability for the RAID $(\alpha=.75)$ and inter-rater reliability $(\kappa=.71)$. The RAID correlated moderately with older adult $(r[32]=.49, p<.01)$ and informant $(r$ [32] $=.36, p<.05)$ ratings of worry as measured by the Penn State Worry Questionnaire (PSWQ) and older adult $(r[32]=.48, p<.01)$ and informant $(r[32]=.46, p<.01)$ rating of general anxiety as assessed by the Geriatric Anxiety Inventory. Additionally, the RAID showed sufficient discriminant validity as it was not correlated with older adultinformant relationship quality (Mutuality Scale), informant report of quality of life (Quality of Life in Alzheimer's Disease scale), or informant report of depression (Geriatric Depression Scale). It was significantly negatively correlated with older adult report of quality of life $(r[32]=-.61, p<.01)$ and positively correlated with report of depression $(r[32]=.52, p<.01)$. Analyses for concurrent validity indicated RAID scores were significantly higher among older adults with a diagnosis of anxiety $(M=17.99, S D$ $=7.15)$ than those without anxiety $(M=10.17, S D=5.62 ; z=-2.79, p<.01)$, and were significantly higher among older adults with a diagnosis of GAD $(M=18.59, S D=7.40)$ than those not diagnosed with $\operatorname{GAD}(M=11.94, S D=6.42 ; z=2.38, p<.05)$. Operating curve analyses revealed that area under the curve was .80 , suggesting the RAID was 
sufficient in distinguishing between older adults with and without anxiety. It was also determined that the cut score of 10 was optimal for diagnostic accuracy with a sensitivity of .90 and specificity of .67 (Snow et al., 2012).

Cohen-Mansfield Agitation Inventory (CMAI). The CMAI is a 29-item measure of agitated behaviors typically displayed by older adults with dementia residing in nursing homes and the community, including physically aggressive, physically nonaggressive, and verbally agitated behaviors (Miller, Snowdon, \& Vaughan, 1995). The behaviors are rated by a formal caregiver (e.g., nursing staff) on a scale from 1 (never) to 7 (several times an hour; Cohen-Mansfield \& Libin, 2004; Cohen-Mansfield, Werner, Watson, \& Pasis, 1995). The presence of agitation is defined as the occurrence of at least one behavior several times a day, and total score or subscale scores may be used as indicators of the presence of agitation (Cohen-Mansfield, 1986; Miller et al., 1995; Weiner et al., 2000).

Finkel, Lyons, and Anderson (1992) completed an evaluation of the psychometric properties of the CMAI as used with long-term care residents. Formal caregivers, inclusive of registered nurses and certified nursing assistants, from all three shifts (day, evening, and night) completed the CMAI for the resident for which they primarily provided care. The CMAI demonstrated internal consistencies (Cronbach's alpha) of .86 (day), .91 (evening), and .87 (night). To assess inter-rater reliability, pairs of formal caregivers were randomly selected for 20 residents across all three shifts. The results of this analysis revealed inter-rater reliability (intraclass coefficient) of .41 for total CMAI score, .66 for the physically aggressive subscale, .26 for the physically non-aggressive subscale, and .61 for the verbally agitated subscale. A moderate correlation existed 
among shifts: day/evening (.51), day/night (.55), and evening/night (.41). The validity of the CMAI when used with nursing home residents was examined using a randomly selected sample of 40 residents for which formal caregivers completed the Behavioral Syndromes Scale for Dementia (BSSD) and the Behave-AD. The BSSD correlated moderately with the day $(r[40]=.52, p<.05)$ and evening $(r[40]=.40, p<.05)$ shifts' ratings based on the CMAI. The Behave-AD had low to moderate correlations with the day $(r[40]=.43, p<.05)$ and evening $(r[40]=.28, p<.05)$ shifts' ratings based on the CMAI. Neither the BSSD, nor the Behave-AD significantly correlated with night shift ratings. Generally, the CMAI appeared to be a reliable and valid assessment of agitated behaviors in nursing homes (Finkel et al., 1992).

A later study of the reliability and validity of the CMAI revealed higher levels of internal consistency, inter-rater reliability, and concurrent validity (Miller et al., 1995). In a randomly selected nursing home, nurses and nursing assistants rated agitated behaviors of residents $(N=100)$ using the CMAI on the day, evening, and night shifts. Internal consistency for the day ( $\alpha=.72)$, evening $(\alpha=.82)$, and night $(\alpha=.63)$ shifts was moderate to high. Twenty residents were randomly selected for which pairs of formal caregivers on the same shift were used to determine inter-rater reliability of the CMAI. Inter-rater reliability for total CMAI scores was high $(r=.82)$, and for the physically aggressive $(r=.85)$, physically non-aggressive $(r=.73)$, and verbally agitated behaviors $(r=.47)$ was moderate to high. Another 20 residents were then randomly selected for which the formal caregivers completed the Nursing Home Behavior Problem Scale (NHBPS) and the Behavioral and Emotional Activities Manifested in Dementia (BEAM-D). The CMAI was moderately to highly correlated with the NHBPS on the day 
$(r[20]=.89, p<.01)$, evening $(r[20]=.95, p<.01)$, and night $(r[20]=.64, p<.01)$

shifts, and the CMAI was highly correlated with the BEAM-D on the day $(r[20]=.91, p$ $<.01)$, evening $(r[20]=.79, p<.01)$, and night $(r[20]=.92, p<.01)$ shifts. Again, the

CMAI demonstrates sufficient reliability and validity when used with nursing home residents with dementia (Miller et al., 1995).

\section{Procedures}

Recruitment. The first step in recruiting the participants involved obtaining approval and cooperation from the long-term care facilities. Facilities in metropolitan Louisville, Kentucky were solicited for participation in the study whereby long-term care facility administrators and social workers were approached via e-mail and sent a brief description of the purpose of the study, the study protocol, and eligibility criteria.

Administrators and social workers were contacted via telephone as follow-up to the initial e-mail.

After obtaining approval from the administrator, a site liaison within the facility was identified. Typically, the site liaison was the facilities' social worker. However, site liaisons also included activities directors and directors of nursing. Either in person or via email or telephone, the site liaison was informed of the purpose of the project, his or her role in the project, eligibility criteria, and information needed to begin the recruitment process. In accordance with a partial Health Insurance Portability and Accountability Act waiver obtained through the Institutional Review Board, the site liaison was asked to create a list of potential participants inclusive of responsible parties, responsible party contact information, resident names, and room/bed numbers that was in line with the eligibility criteria discussed above. The site liaisons were provided with a checklist to aid them in 
identifying potential participants and help ensure recruitment of eligible participants. Lists of eligible participants were obtained from site liaisons through secure e-mail, fax, or in person.

Informed consent. Obtaining informed consent from older adults in long-term care facilities was sometimes challenging due to a lack of understanding of the purpose of research and general skepticism surrounding involvement in a research study. When obtaining informed consent from the resident, the purpose and process of providing informed consent was verbally explained to each potential participant. The purpose and process of the research study, including what information would be gathered, when the information would be gathered, from whom the information would be gathered, the measures that would be completed, benefits and risks, and issues of privacy and confidentiality, were also described and reiterated as needed. The participants were informed that participation was voluntary, and any questions about the procedures were addressed fully and truthfully. To ensure the resident understood the informed consent and research process, he or she was asked to complete a brief test to assess comprehension of consent. Based on Buckles and colleagues' (2003) brief test designed to assess older adults' (with and without dementia) understanding of the consent process, this eightquestion test asked the resident to describe in his or her own words the purpose, process, risks, and benefits of the study, as well as the meaning of "confidential" and "voluntary." The informed consent process was considered an iterative process during which all investigators engaged the potential participant in open conversation about the research being conducted both before and after the test of comprehension to consent. In the event that the investigator felt the potential participant's ability to provide informed consent was 
questionable, the resident's responsible party was contacted for informed consent by proxy in accordance with the procedure outlined below.

In addition to obtaining a list of potential participants from the site liaison, a list of the responsible parties for residents was acquired. Individuals residing in long-term care facilities, regardless of their decision-making ability, usually have responsible parties with whom long-term care staff consults regarding residents' care. Often the responsible parties are spouses or adult children, but may also be members of residents' extended family or another trusted individual. Responsible parties may also be powers of attorney or appointed guardians. Involving responsible parties in the consent process was imperative when recruiting participants with cognitive impairment, given that individuals with cognitive impairment were not always be able to provide informed consent. Therefore, the responsible parties of the residents identified as eligible for the participation in the study, but unable to provide informed consent (e.g., could not describe the purpose, process, risks, and benefits of the study, despite education from the investigators), were contacted via mail with a letter describing the purpose of the research study, a copy of the informed consent by proxy and assent forms, and a telephone number to contact with questions or concerns about the research and informed consent process. This process allowed responsible parties to "opt out" of the study. Though it was recognized that contacting the responsible parties for consent did not imply that potential participants were not capable of deciding to participate in the study and participation was not solely be based on the responsible party's decision, the process of contacting responsible parties for informed consent by proxy was done out of respect for concerns and wishes of the responsible parties. Certain situations, including recruiting residents in memory care/dementia units and those with guardians, 
precluded the informed consent process. In these situations, responsible parties were first contacted for informed consent by proxy, and then written resident assent was obtained.

In sum, many long-term care residents with dementia are capable of providing informed consent. If the resident was capable of providing consent, then he or she provided informed consent and completed the consent form. In the case that the resident was not able to provide informed consent, then his or her responsible party was consulted and consent by proxy was obtained followed by obtaining written assent from the participant.

Completion of measures. Administration of screening and assessment instruments is standard procedure in long-term care facilities. Regular screening of cognitive functioning and psychopathology is part of routine care in facilities long-term care facilities. Thus, screening for symptoms of anxiety and mental health history and rating affect was neither out of the ordinary for residents or staff, nor particularly disruptive of daily routine. Overall, screening was not considered invasive or above minimal risk by residents or staff.

After informed consent/assent was obtained for the eligible resident, a doctoral or trained undergraduate student completed the RAID, mental health history screening questions, and ARS with the resident. All measures were read aloud to the resident, unless the resident preferred to complete the measure him- or herself. The order of administration was randomized to minimize potential response bias using a Web-based random number generator. Additionally, participants' demographic information, behavior, functional status, active disease diagnosis, medications, special treatments and 
procedures, BIMS, and PHQ-9 was obtained and recorded from the residents' most recent MDS.

Certified Nursing Assistants (CNAs) responsible for the day-to-day care of the older adult participant provided informant responses to the RAID and CMAI. CNAs consistently spend the most time per day with residents than other members of nursing staff, and have been shown to be fairly accurate and unbiased in their ratings of memory and behavior problems exhibited by nursing home residents (Allen et al., 2003; Horn, Buerhaus, Bergstron, \& Smout, 2005; Seblega et al., 2010). A doctoral or trained undergraduate student completed the RAID and the CMAI with the CNA. Again, the order of administration was randomized to minimize potential response bias.

Also, the responsible party identified through the recruitment process was contacted by telephone to complete the mental health history screening questions. Completing the mental health history screen with residents' responsible parties was important to corroborate mental health history and management, especially if the participant is cognitively impaired (Starkstein, Jorge, Mizrahi, \& Robinson, 2006).

\section{Statistical Power}

Ten to fifteen participants per variable (including measure subscales) has been used as a guiding rule for determining an appropriate sample size for factor analyses (Field, 2005), resulting in a sample size of 150 to 225 participants. At the same time, Stevens (2002) asserts that a sample is adequate if there are at least two participants for each assessment instrument item. Thus, a minimum sample size of 184 was appropriate when using the 7-item BIMS, 9-item PHQ-9, 19-item mental health screening questions, 18-item RAID, 10-item ARS, and 29-item CMAI. A more liberal estimate of the sample 
size needed to complete a factor analysis was 3 (Bollen, 1989) or 5 to 10 (Bentler, 1990) participants per variable, yielding a minimal sample size of 30 to 100 participants. This estimate was more achievable given the inherent difficulties of conducting research in long-term care facilities. With this sample size more stringent guidelines were applied to consider factor loadings statistically significant (Field, 2005).

An analysis of variance (ANOVA) was completed to test the second and third hypotheses. Limited published research is available on assessing anxiety in older adults with dementia. An effect size of .28 was determined from a factorial ANOVA completed with data gathered for a dissertation evaluating James' (1999) cognitive model of anxiety in nursing home residents with dementia (Tennyson, 2003). G*Power 3.1.2 was used to calculate the sample size needed to determine the main effects and the interaction for the 2x3 ANOVA given $\alpha$ of .05 and power of .80 , producing a sample size of 102 for the first main effect, 126 for the second main effect, and 126 for the interaction. Due to challenges in recruitment, this ideal $N$ was not attained. Consequently, the study is under-powered for these analyses.

\section{Analyses}

Descriptive statistics. Descriptive statistics for the demographic and independent and dependent variables were performed using IBM SPSS Statistics Version 20. Oneway ANOVA and Chi-square analyses were conducted to ascertain potential differences between eligible and ineligible participants; according to race, sex, marital status, and place from which resident had entered the long-term care facility; and among participants with dementia and mental health diagnoses as listed in their MDS 3.0. 
Approach to Confirmatory Factor Analysis (CFA). Statistical analyses for the CFA described below were completed using IBM SPSS AMOS Version 20 (AMOS). As noted above, the relationships among anxiety and depression and anxiety and agitation have been established for community-dwelling older adults with dementia and those living in long-term care facilities. The relationship between agitation and depression has not been established for older adults with dementia living in long-term care, nor has the relationship among anxiety, depression, and agitation for this population. The purpose of the confirmatory factor analyses was to explore possible factor solutions for the relationships among anxiety, depression, and agitation in this population.

To examine measure invariance, three measurement models were estimated as follows: (1) a depression latent factor with the variables PHQ-9, ARS positive affect subscale, and ARS negative affect subscale; (2) an anxiety latent factor with the variables ARS negative affect subscale, the RAID subscales, and the CMAI subscales; and (3) an agitation latent factor with the variables RAID worry, motor tension, and apprehension and vigilance subscales, and the CMAI subscales loading onto them. Measurement models were assessed for global fit using the Chi-square goodness-of-fit test, comparative fit index (CFI), incremental fit index (IFI), Tucker-Lewis Index (TLI), and root mean square error of approximation (RMSEA). Values greater than or equal to .90 for the fit indices and an RMSEA less than or equal to .06 were considered acceptable ( $\mathrm{Hu} \&$ Bentler, 1999).

Following the measurement models, it was determined a single factor CFA should be completed to the test the alternative hypothesis that anxiety, depression, and agitation in older adults with dementia residing in long-term care facilities represented a single 
overarching construct of distress. The single factor model was estimated in which AMOS was provided with the single latent factor of distress and the variables specified in the measurement models loading onto it. Additionally, the error terms for physically non-aggressive and verbal agitation and PHQ-9 and worry were correlated in line with suggested modification indices. The latent factor was scaled by fixing one factor loading to 1.0. The global fit of one factor model was analyzed, as well as the factor loadings for their acceptability. A second one factor model of distress was estimated using the CNA responses to the RAID. This model followed the single factor model using resident responses to the RAID for variable loading. As indicated in the modification indices for this model, the following error terms were correlated: physically non-aggressive and verbal agitation, physically aggressive and verbal agitation, worry and negative affect, PHQ-9 and negative affect, and PHQ-9 and positive affect. Again, the latent factor was scaled by fixing one factor loading to 1.0 . The global fit of the one factor model was examined, as well as the factor loadings for their acceptability. Finally, the single factor model using the resident responses to the RAID was compared to the single factor model using the CNA responses to the RAID for superiority of fit.

Analysis of Variance (ANOVA). In order to test the second and third hypotheses, factorial ANOVAs were completed for each hypothesis using IBM SPSS Version 20. For the second hypothesis, it was supposed that anxiety may have an effect on agitation, but that this effect may be different for long-term care residents with different levels of global cognitive functioning. Accordingly, anxiety (absent or present using RAID cut score of 11 as rated by both the resident and the primary CNA) and cognitive functioning (intact/borderline, moderate impairment, severe impairment) were 
independent variables and agitation as measured by the CMAI were the dependent variable. For this analysis the main effects of anxiety and cognitive functioning, as well as the interaction of anxiety and cognitive functioning, were examined. For the third hypothesis, it was supposed that history of mental illness may have an effect on current anxiety, but that this effect may be different for long-term care residents with different levels of global cognitive functioning. Therefore, mental health history (general mental health, history of either a mood or anxiety disorder, history of depression, or history of anxiety as indicated by the Vahia et al. [2010] and SCID screening questions) and cognitive functioning (intact/borderline, moderate impairment, severe impairment) were considered independent variables and current anxiety as measured by the RAID were the dependent variable. Analyses were run for both resident and CNA responses to the RAID. Like the analysis for the second hypothesis, the main effects of mental health history and cognitive functioning and the interaction of these independent variables were examined. Additionally, descriptive statistics, contrasts, post-hoc analyses, and homogeneity of variance were examined for each analysis. 


\section{CHAPTER 3}

\section{RESULTS}

\section{Data Transformation}

Exploration of the data revealed measures for negative affect, depression, anxiety, and agitation were significantly non-normal. The measure for positive affect however, was normally distributed $(D[65]=.09, p>.05)$. Examination of the distributions of the measures for negative affect, depression, anxiety, and agitation revealed positively skewed data. For this reason, base 10 logarithmic transformation with the addition of the constant "1" was used on the data. See Table 1 for the test of normality.

\section{Sample Demographics and Descriptive Statistics}

Eighty-three long-term care residents agreed to participate in the study. Of the 83 residents who agreed to participate in the study, 65 were eligible to participate. Reasons for ineligibility included being under the age of $65(n=4)$ and not having a dementia diagnosis indicated in their medical charts $(n=18)$. Eligible and ineligible participants

did not differ in regard to sex $\left(\chi^{2}[1, N=83]=3.73, p \geq .05\right)$, race $\left(\chi^{2}[1, N=83]=.23 . p\right.$ $=.63)$, and place from which resident entered facility $\left(\chi^{2}[5, N=83]=2.29, p=.81\right)$. Participants differed according to marital status $\left(\chi^{2}[3, N=83]=8.79 . p<.05\right)$, with more ineligible participants divorced, and length of stay ( $[81]=-2.24, p<.05)$, such that ineligible participants had resided for a shorter period of time in their facilities. As expected, ineligible participants were significantly younger $(F[1,83]=11.28, p<.01)$ and had significantly less global cognitive impairment than eligible participants $(F[1,83]$ 
$=6.05, p<.05)$. No significant differences were found between eligible and ineligible participants in regard to experience of positive $(F[1,83]=.06, p=.81)$ or negative affect $(F[1,83]=.78, p=.38)$, severity of anxiety as rated by the resident $(F[1,83]=$ $.23, p=.63)$ or CNAs $(F[1,83]=.95, p=.33)$, exhibition of agitated behaviors $(F[1$, $83]=.24, p=.63)$, or severity of depression $(F[1,83]=.43, p=.51)$. Moreover, eligible and ineligible participants did not differ significantly in regard to general mental health history $\left(\chi^{2}[1, N=83]=.08 \cdot p=.78\right)$, history of mood or anxiety disorder $\left(\chi^{2}[1, N=83]\right.$ $=.54 \cdot p=.46)$, history of depression $\left(\chi^{2}[1, N=83]=.01 \cdot p=.92\right)$, or history of anxiety $\left(\chi^{2}[1, N=83]=.01 . p=.90\right)$. See Table 2 for demographic characteristics and Table 3 for descriptive statistics of measures collected for this study for ineligible participants.

Of the 65 eligible participants, the majority of the residents were female $(73.8 \%)$, European American (87.7\%), and widowed (66.2\%). The mean age of participants was $82.42(S D=7.84)$ with $34.8 \%$ of the participants age 85 or older. Most had entered from an acute hospital stay (86.2\%) and had been residing in their long-term care facility for approximately 24 months $(M=23.89, S D=20.70)$. The most frequent medical diagnosis was hypertension (86.2\%), though other medical diagnoses (i.e., diabetes mellitus [30.8\%], hyperlipidemia [24.6\%], and diseases of the respiratory system [16.9\%]) were fairly common. Overall, the sample collected for this study aligned with national estimates of the long-term care population (Jones et al., 2009).

According to data collected from residents' most recent MDS, $33.8 \%$ of the participants had a diagnosis of Alzheimer's disease and $72.3 \%$ had a diagnosis of dementia. Mental health diagnoses were prevalent among this population. Two-thirds of the participants (66.2\%) had a diagnosis of depression listed in their charts, while slightly 
over one-third (35.4\%) had a diagnosis of anxiety. Fewer participants had a diagnosis of more severe mental illness, including bipolar disorder (7.7\%), psychotic disorder (4.6\%), and schizophrenia (9.2\%). Antidepressants were the most commonly prescribed psychoactive medication (67.7\%), followed by anxiolytics (24.6\%) and antipsychotics (24.6\%). None of the participants had received psychological therapy for at least 15 minutes in the last 7 days.

Residents did not differ on anxiety, depression, affect, or cognitive status by race, sex, marital status, or place from which residents entered their facilities, with one exception. Anxiety as rated by the residents' primary CNAs was significantly higher among participants who had entered their facility from inpatient rehabilitation $(M=$ $19.50, S D=6.36$ v. $M=6.66, S D=7.03$ [entered from acute hospital stay]; $F[5,65]=$ $2.73, p<.05$ ), suggesting the place from which the resident entered may have contributed to increased symptoms of anxiety as has been demonstrated by previous research (Thomasma et al., 1990).

Several significant differences arose between participants with and without mental health disorder diagnoses as indicated in their most recent MDS. Residents with a diagnosis of depression had significantly higher CMAI scores $(M=11.49, S D=12.21 ; F$ $[1,65]=5.34, p<.05)$, and RAID scores as reported by CNAs $(M=7.95, S D=7.95 ; F$ $[1,65]=7.75, p<.01)$, although not higher for self-rated RAID scores. BIMS scores were also higher among participants with a depression diagnosis $(M=10.35, S D=4.21$; $F[1,65]=7.80, p<.01)$ than residents who do not have a depression diagnosis listed in their charts $(M=6.91, S D=4.36)$. As anticipated, those individuals with a diagnosis of anxiety had significantly higher PHQ-9 scores $(M=2.87, S D=2.65 ; F[1,65]=4.23, p$ 
$<.05)$ than those without an anxiety diagnosis $(M=1.98, S D=3.14)$. But, contrary to what was expected, no other significant differences on measures of anxiety, agitation, or cognitive functioning were found between participants with and without anxiety diagnoses.

See Table 4 for demographic characteristics of the eligible participants, and Table 5 for descriptive statistics of measures related to the hypotheses.

\section{Hypothesis 1}

Bivariate correlations. Review of the bivariate correlations among measures and their subscales revealed several statistically correlations. Specifically, the ARS positive affect subscale was significantly negatively correlated with the resident RAID total score $(r[65]=-.25, p<.05)$, the RAID apprehension and vigilance subscale $(r[65]=-.40, p<$ $.01)$, and the PHQ-9 total score $(r[65]=-.32, p<.01)$. The ARS negative affect subscale was significantly positively correlated with the RAID total score $(r[65]=.43, p<.01)$ and its worry $(r[65]=.37, p<.01)$, apprehension and vigilance $(r[65]=.40, p<.01)$, motor tension $(r[65]=.33, p<.01)$, and autonomic hypersensitivity $(r[65]=.31, p<$ $.05)$ subscales; as well as the PHQ-9 total score $(r[65]=.32, p<.01)$. The RAID total score and subscale scores were significantly correlated with the exception of the RAID autonomic hypersensitivity and motor tension subscales $(r[65]=.17, p=.17)$. Similarly, the CMAI total score and subscale scores were significantly correlated with the exception of the CMAI physically aggressive and physically non-aggressive agitation $(r[65]=.15$, $p=.25$ ). A number of significantly positive correlations existed among resident RAID and CMAI subscale scores, including the RAID worry and CMAI verbal agitation subscale $(r[65]=.29, p<.05)$; the RAID apprehension and vigilance and CMAI 
physically aggressive agitation $(r[65]=.29, p<.05)$ and physically non-aggressive agitation subscales $(r[65]=.26, p<.05)$; and the RAID motor tension and CMAI verbal agitation subscales $(r[65]=.30, p<.05)$. The RAID autonomic hypersensitivity subscale was not significantly correlated with any of the CMAI subscales. Lastly, the PHQ-9 total score was significantly correlated with the RAID apprehension and vigilance $(r[65]=.31, p<.05)$ and motor tension $(r[65]=.29, p<.05)$ subscales. The negative affect and cognition factor of the PHQ-9 was not significantly correlated with any measures or their subscales other than the total PHQ-9 $(r[65]=.75, p<.01)$. See Table 8 in the Appendices for bivariate correlations of the measures and their subscales for residents.

Bivariate correlations were also analyzed for the CNA RAID total and subscale scores. As with the resident RAID total and subscale scores, the CNA RAID total and subscale scores were significantly correlated. Multiple significantly positive correlations were found between the RAID and CMAI subscale scores, including between the CMAI physically non-aggressive and RAID worry $(r[65]=.33, p<.01)$ and motor tension $(r$ $[65]=.35, p<.01)$ subscales and among the CMAI verbal agitation and RAID worry $(r$ $[65]=.55, p<.01)$, apprehension and vigilance $(r[65]=.58, p<.01)$, motor tension $(r$ $[65]=.41, p<.01)$, and autonomic hypersensitivity $(r[65]=.28, p<.05)$ subscales. No significant correlations were found among the CMAI physically aggressive subscale and the RAID subscales. Finally, the PHQ-9 and the negative affect/cognition factor of the PHQ-9 were not significant correlated with the RAID subscales. See Table 9 in the Appendices for bivariate correlations of the measures and their subscales for CNAs. 
Measurement models. Three measurement models were estimated before specifying the hypothesized three factor model. The first measurement model was estimated as the latent factor depression with the ARS positive and negative affect subscales and PHQ-9 total score loading onto it. However, the model was inadmissible $(d f=0)$. For this reason, an additional conceptually relevant variable (RAID worry subscale) was added to the model. The results of this model indicated insufficient fit $\left(\chi^{2}\right.$ $[2, N=65]=6.01, p=.05 ; \mathrm{CMIN} / \mathrm{df}=3.01, \mathrm{IFI}=.83, \mathrm{TLI}=.39, \mathrm{CFI}=.80, \mathrm{RMSEA}=$ .18), suggesting that these measures do not collectively represent a coherent construct of depression in the current sample. A review of the bivariate correlations revealed a significant positive correlation between PHQ-9 total score and the RAID apprehension and vigilance subscale (see Table 8 in Appendices). The measurement model was again estimated with the variable RAID apprehension and vigilance loading onto it. The results indicated substantially improved fit $\left(\chi^{2}[2, N=65]=1.38, p=.50 ; \mathrm{CMIN} / \mathrm{df}=.69, \mathrm{IFI}=\right.$ $1.02, \mathrm{TLI}=1.07, \mathrm{CFI}=1.00, \mathrm{RMSEA}=.00$ ). The improvement in model fit with the addition of the RAID apprehension and vigilance variable suggests the hypothesized measures were not necessarily indicative of the traditional conceptualization of depression. Rather, the variables in the second measurement model for depression may be more indicative of a latent factor of psychological distress in this sample, which coincides with the proposed alternative model of general distress.

The second measurement model was estimated as the latent factor anxiety with the ARS negative affect subscale; the resident RAID worry, apprehension and vigilance, motor tension, and autonomic hypersensitivity; and CMAI physically aggressive, physically non-aggressive, and verbal agitation subscales loading onto it. Analyses 
demonstrated good global fit $\left(\chi^{2}[20, N=65]=28.27, p=.10\right)$. Examination of the modification indices indicated linking the error terms for the CMAI physically nonaggressive and verbally agitated subscales would improve model fit. Results of this analysis indicated good fit $\left(\chi^{2}[19, N=65]=20.07, p=.39 ; \mathrm{CMIN} / \mathrm{df}=1.06, \mathrm{IFI}=.99\right.$, $\mathrm{TLI}=.98, \mathrm{CFI}=.99, \mathrm{RMSEA}=.03)$.

The third measurement model was estimated as the latent factor agitation with the RAID worry, apprehension and vigilance, and motor tension subscales and CMAI physically aggressive, physically non-aggressive, and verbal agitation subscales loading on to it. Analyses demonstrated good fit $\left(\chi^{2}[9, N=65]=13.08, p=.39\right)$. Like the anxiety measurement model, review of the modification indices indicated correlating the error terms for the CMAI physically non-aggressive and verbally agitated subscales would improve model fit. Results of this analysis indicated good fit $\left(\chi^{2}[8, N=65]=\right.$ $7.07, p=.53 ; \mathrm{CMIN} / \mathrm{df}=.88, \mathrm{IFI}=1.02, \mathrm{TLI}=1.04, \mathrm{CFI}=1.00, \mathrm{RMSEA}=.00)$.

Given the results of the measurement model analyses, it was determined that a three factor model with the latent factors of depression, anxiety, and agitation as typically theoretically viewed and as indicated by the literature on these constructs as they pertain to older adults likely did not best represent the relationship among the variables measured in this study for the current sample (Cook et al., 2004; Mineka et al., 1998; Teachman et al., 2007). Although a two factor model of anxiety and agitation was considered in which the indicators of depression were excluded, research on the relationships among depression, anxiety, and agitation in the studied sample indicated otherwise, given the well-document overlap between depression and anxiety. Consequently, the three factor model approach to the data was terminated and the alternative model estimating general 
distress as has been demonstrated in community-dwelling samples of older adults was pursued (Meeks et al., 2002).

Single factor model representing general distress. An initial hierarchical three factor model was estimated in which AMOS was provided with the latent factor of distress (see Figure 1) with PHQ-9 total score, ARS positive and negative affect subscales, and RAID autonomic hypersensitivity subscale loading on to it, as well as two endogenous variables: (1) cognitive/verbal (RAID worry and CMAI verbally agitated subscales) and (2) behavioral (RAID motor tension and apprehension and vigilance and CMAI physically aggressive and non-aggressive subscales). However, the coefficient for the endogenous variable "behavioral" was greater than 1 (1.12). Modification indices suggested linking the error terms for physically non-aggressive and verbal agitation. Still, the coefficient for the endogenous variable "behavioral" was greater than 1 (1.09), suggesting there may be too much shared variance with this variable (see Figure 2). Therefore, the hierarchical three factor model was revised as the overarching latent factor of distress estimated with all variables loading directly onto it and the error terms for CMAI physically non-aggressive and verbal agitation correlated as indicated by the measurement models. Results from this single factor model achieved acceptable fit $\left(\chi^{2}\right.$ $[33, N=65]=39.72, p=.23 ; \mathrm{CMIN} / \mathrm{df}=1.17, \mathrm{IFI}=.94, \mathrm{TLI}=.91, \mathrm{CFI}=.93, \mathrm{RMSEA}$ $=.05$ ). Modification indices suggested fit would be improved if the error terms for the RAID worry subscale and PHQ-9 total score were linked. Results from this modified model indicated improved fit $\left(\chi^{2}[33, N=65]=34.48, p=.40 ; \mathrm{CMIN} / \mathrm{df}=1.05, \mathrm{IFI}=\right.$ $.96, \mathrm{TLI}=.98, \mathrm{CFI}=.98, \mathrm{RMSEA}=.03$ ). See Figure 3 for a depiction of this model. Factor loadings were positive, ranging from low to medium (.25 to .69), with the 
exception of positive affect with a low factor loading of -.36 , and were statistically significant from zero. See Table 6 for factor loadings for the model estimated with the resident responses to the RAID.

A second single factor model was estimated using the CNA RAID subscale scores based on the model estimated using the resident RAID subscale scores (see Figure 4). Results of this model demonstrated poor global fit $\left(\chi^{2}[34, N=65]=63.74, p<.01\right)$. In line with the modification indices the error terms for PHQ-9 total score and ARS positive and negative affect subscales were correlated as were the error terms for the ARS negative affect and RAID worry subscales. The CMAI physically aggressive and verbal agitation subscales were also correlated. Results revealed improved model fit $\left(\chi^{2}[30, N\right.$ $=65]=42.97, p=.06 ; \mathrm{CMIN} / \mathrm{df}=1.43, \mathrm{IFI}=.91, \mathrm{TLI}=.85, \mathrm{CFI}=.90, \mathrm{RMSEA}=.08)$. For this analysis, factor loadings were generally positive (.08-.83), ranging from low to high, with the exception of positive affect (-.08), and were statistically significant from zero. See Table 7 for factor loadings for the model estimated with the CNA responses to the RAID.

Differences and similarities exist in the factor loadings of each of the variables between the resident and CNA models. Notably, the factor loadings for PHQ-9, positive affect, and negative affect were lower for the CNA model than the resident model, the factor loadings from the RAID subscales were generally higher for this model, and the CMAI subscale scores varied more than the resident model. However, in both models the RAID apprehension and vigilance subscale was the greatest contributor to the latent factor. Overall, it appears as though the single factor model inclusive of resident RAID 
subscale scores represents better global fit than the model utilizing CNA RAID subscale scores.

\section{Hypothesis 2}

To examine the hypothesis that the relationship between anxiety as measured by the RAID and agitation as measured by the CMAI was moderated by global cognitive functioning as measured by the BIMS, two two-way repeated measures ANOVAs were completed. The first ANOVA used the RAID total scores as derived from long-term care residents' responses, while the second ANOVA used the RAID total scores as taken from their primary CNAs' responses. The results of the first ANOVA revealed a significant main effect of anxiety on the frequency of agitated behaviors $(F[1,59]=4.52, p<.05$, $\left.\eta^{2}=.07\right)$. Further examination of the means showed CMAI scores were significantly higher for long-term care residents with clinically significant anxiety (i.e., RAID total score greater than or equal to $11 ; M=13.21, S D=11.82)$ than those whose scores suggested an absence of anxiety $(M=7.85, S D=9.22)$. There was no significant main effect of global cognitive functioning $\left(F[2,59]=1.87, p=.16, \eta^{2}=.06\right)$. Moreover, there was not a significant interaction effect between anxiety and global cognitive

functioning $\left(F[2,59]=.22, p=.81, \eta^{2}=.01\right)$. Accordingly, global cognitive functioning does not appear to moderate the relationship between current anxiety as reported by longterm care residents and the exhibition of agitation. Similar results were found for the second analysis using CNA responses to the RAID. However, the results must be interpreted with caution, as Levene's test for homogeneity of variance was significant $(F$ $[5,59]=5.50, p<.001)$. A significant main effect of anxiety was found $(F[1,59]=$ $13.41, p<.01, \eta^{2}=.19$ ) indicating higher frequency of agitated behaviors among long- 
term care residents with clinically significant anxiety $(M=20.23, S D=11.42)$ than those whose scores suggested an absence of anxiety $(M=6.71, S D=7.99)$. There was no significant main effect of global cognitive functioning $\left(F[2,59]=.30, p=.74, \eta^{2}=.01\right)$. Furthermore, there was no significant interaction effect between anxiety and global cognitive functioning $\left(F[2,59]=.17, p=.84, \eta^{2}=.01\right)$. Thus, global cognitive functioning does not appear to moderate the relationship between resident anxiety as assessed by their primary CNAs and frequency of agitation.

\section{Hypothesis 3}

In order to test the hypothesis that mental health history was predictive of current anxiety in long-term care residents with dementia, but moderated by global cognitive functioning, eight two-way repeated measures ANOVAs were completed. As with the second hypothesis, analyses were run first using RAID total scores from long-term care residents' responses as dependent variables. Separate analyses were run with different mental health history indices as independent variables, including general mental health history (i.e., affirmative response to at least one Vahia et al. [2010] self-report item of mental health history), history of mood or anxiety disorder (i.e., affirmative response to at least one SCID screening question for depression or anxiety), history of depression, and history of anxiety.

There was a significant main effect for general mental health history on the severity of current anxiety $\left(F[1,59]=6.23, p<.05, \eta^{2}=.10\right)$. Long-term care residents who had been diagnosed with a mental health disorder or had received mental health services in the past endorsed greater anxiety severity $(M=10.92, S D=7.81)$ than those who did not have a general mental health history $(M=6.20, S D=4.99)$. The main effect 
of global cognitive functioning on current anxiety was not significant $(F[2,59]=.00, p=$ $.99, \eta^{2}=.00$ ). The interaction effect between general mental health history and global cognitive functioning on current anxiety was also non-significant $(F[2,59]=.45, p=$ $\left..64, \eta^{2}=.02\right)$.

There was a significant main effect for history of mood or anxiety disorder $(F[1$, $59]=13.95, p<.01, \eta^{2}=.19$ ). Those residents who confirmed past depression and/or anxiety reported greater anxiety severity $(M=9.06, S D=6.61)$ than those who did not $(M=3.00, S D=3.08)$. There was no significant main effect of cognitive functioning $(F$ $[2,59]=.04, p=.97, \eta^{2}=.00$ ) or interaction effect between history of depression and/or anxiety and cognitive functioning $\left(F[2,59]=.71, p=.50, \eta^{2}=.02\right)$. Again, there appears to be no moderating effect of global cognitive functioning on the relationship between history of depression and/or anxiety and current anxiety.

An analysis of the relationship between history of depression alone and current anxiety demonstrated a significant main effect $\left(F[1,59]=18.97, p<.01, \eta^{2}=.24\right)$. Residents with a history of depression reported greater frequency and intensity of anxiety $(M=11.54, S D=7.11)$ than those without a history of depression $(M=5.22, S D=4.53)$. As with the analyses discussed above, there was no significant main effect of cognitive functioning $\left(F[2,59]=.25, p=.78, \eta^{2}=.01\right)$, or interaction effect between history of depression and cognitive functioning $\left(F[2,59]=1.95, p=.15, \eta^{2}=.06\right)$. Cognitive functioning does not appear to moderate the relationship between history of depression and current anxiety.

Finally, there was a significant main effect of history of anxiety alone on current anxiety $\left(F[1,59]=24.36, p<.01, \eta^{2}=.29\right)$. As with residents with a history of 
depression, residents with a history of anxiety reported greater severity $(M=9.74, S D=$ 6.70) than those without a history of anxiety $(M=3.58, S D=3.41)$. There was no significant main effect of cognitive functioning $\left(F[2,59]=.29, p=.75, \eta^{2}=.01\right)$, or interaction effect between history of anxiety and cognitive functioning $(F[2,59]=2.70$, $p=.08, \eta^{2}=.08$ ). The relationship between history of anxiety and current anxiety does not appear to be moderated by cognitive functioning.

In addition to the two-way repeated measures ANOVAs completed with the resident responses to the RAID, four two-way repeated measures ANOVAs were completed with the residents' primary CNAs' response to the RAID as CNAs have been demonstrated to spend the most time with long-term care residents and provide reliable ratings of potential pathology in this population. There was no significant main effect for general mental health history on the severity of current anxiety as rated by the CNAs ( $F$ $[1,59]=.12, p=.73, \eta^{2}=.00$ ). The main effect of global cognitive functioning on current anxiety was not significant $\left(F[2,59]=2.24, p=.12, \eta^{2}=.07\right)$, nor was the interaction effect between general mental health history and global cognitive functioning on current anxiety $\left(F[2,59]=.59, p=.56, \eta^{2}=.02\right)$.

There was a significant main effect was found for history mood or anxiety disorder $\left(F[1,59]=12.45, p<.01, \eta^{2}=.17\right)$. Those residents who confirmed past depression and/or anxiety had greater anxiety severity noted by CNAs $(M=7.58, S D=$ 7.50) than those who did not $(M=1.83, S D=1.40)$. There was no significant main effect of cognitive functioning $\left(F[2,59]=.51, p=.60, \eta^{2}=.02\right)$ or interaction effect between history of depression and/or anxiety and cognitive functioning $(F[2,59]=.89, p=.42$, $\eta^{2}=.03$ ). Depression history alone did not show a significant relationship with CNA- 
rated anxiety symptoms $\left(F[1,59]=2.46, p=.12, \eta^{2}=.40\right)$. Also, there was no significant main effect of cognitive functioning $\left(F[2,59]=2.45, p=.10, \eta^{2}=.08\right)$ or interaction effect between history of depression and cognitive functioning $(F[2,59]=$ $\left..09, p=.92, \eta^{2}=.00\right)$. Cognitive functioning does not appear to moderate the relationship between history of depression and current anxiety. However, similar to the results found with the residents' responses, there was a significant main effect of history of anxiety alone on current anxiety $\left(F[1,59]=18.64, p<.01, \eta^{2}=.24\right)$. As with residents with a history of depression, residents with a history of anxiety reported greater anxiety severity $(M=8.13, S D=7.76)$ than those without a history of anxiety $(M=2.63$, $S D=2.95)$. There was no significant main effect of cognitive functioning $(F[2,59]=$ $2.91, p=.06, \eta^{2}=.09$ ), or interaction effect between history of anxiety and cognitive functioning $\left(F[2,59]=.65, p=.53, \eta^{2}=.02\right)$. The relationship between history of anxiety and current anxiety does not appear to be moderated by cognitive functioning. 


\section{CHAPTER 4}

\section{DISCUSSION}

This study examined the relationships among anxiety, depression, and agitation in older adults with dementia residing in long-term care, as well as the roles of global cognitive functioning and mental health history in the experience of anxiety in this population. The primary hypothesis was that anxiety, depression, and agitation would represent separate, but related constructs in long-term care residents with dementia. Additionally, it was supposed that global cognitive functioning would moderate the relationships between experience of current anxiety and agitation and between mental health history and current anxiety.

\section{Structural Representation of Depression, Anxiety, and Agitation}

Understanding the relationships among depression, anxiety, and agitation is of theoretical and clinical importance given that little is known about the structural differentiation of anxiety and depression and anxiety and agitation in this population the differentiation of which could lead to improved care and quality of life. Dementia, depression, and anxiety are the most common psychiatric disorders among older adults in long-term care (Seitz et al., 2010). Dementia, depression, and anxiety commonly cooccur and, coupled with agitation that is also frequent in this population, the increasing number of long-term care residents with dementia and mental health disorders creates a challenge for providing quality care (Lenze \& Wetherell, 2011). Much research has found that anxiety arises within the experience of depression and vice versa, and has also 
determined symptom overlap in long-term care residents with dementia (Smallbrugge, Jongenelis, et al., 2005; Ballard et al., 1994; Ferretti et al., 2001; Gibbons et al., 2006; Harwood et al., 1998; Mack et al., 1999; Mega et al., 1996; Tariot et al., 1995; Teri et al, 1999; Teri et al, 1992). The extant literature suggests two conceptualizations of anxiety and depression in this population. The first characterizes anxiety and depression as being related by negative affect, but differentiated by the presence of physiological symptoms and low positive affect as has been seen in younger adults and populations of communitydwelling and psychiatric outpatient older adults without dementia (Cook et al., 2004; Mineka et al., 1998). The second conceptualizes anxiety and depression as a general experience of distress associated with the process of dementia, which has also been found in community-dwelling populations of older adults without dementia (Meeks et al., 2003). Additionally, anxiety often co-occurs with agitation in long-term care residents with dementia. Anxiety has been linked to increased agitation, leading many to conceptualize agitation as the behavioral manifestation of anxiety as dementia progresses (Cohen-Mansfield \& Billig, 1986; Ferretti et al., 2001; Mintzer \& Brawman-Mintzer, 1996; Mega et al., 1996; Ownby et al., 2000; Porter et al., 2003). Although they co-occur and the presence of anxiety may exacerbate agitation, agitation has also been conceptualized as a distinct construct distinguished by behaviors driven by unmet needs precipitated by a variety of biological, psychological, social, and environmental antecedents (Kong, 2005). As with anxiety and depression, anxiety and agitation in older adults with dementia may represent separate, but related constructs. Furthermore, the addition of agitation to the conceptualization of the relationship between anxiety and 
depression may explicate the complexity of the constructs in long-term care residents with dementia.

Individual measurement models. Based on theoretical and empirical knowledge of depression among older adults, including those in long-term care, it was expected that measures of affect and depression would correlate. These measures, however, did not correlate exactly as anticipated in this sample. Positive affect and negative affect were not significantly correlated with one another. Positive affect was significantly correlated with a measure of depression, as well as the anxiety subscale of apprehension and vigilance, in the expected direction. As expected, negative affect demonstrated significant positive correlation with the measure of depression, as well as the anxiety measure and its subscales. The measure of depression was significantly correlated with the anxiety measure, overall, and the apprehension and vigilance and motor tension subscales of this measure. These findings were consistent with findings by other researchers who showed significant low to moderate correlations among negative affect, positive affect, and anxiety in community-dwelling and outpatient psychiatric older adults (Cook et al., 2004; Teachman et al., 2007).

Despite promising correlations consistent with past research, the measurement model for depression completed in anticipation of the confirmatory factor analyses proved discouraging. The measurement model for depression was inadmissible, suggesting that the indicators of positive affect, negative affect, and depression were not indicative of the traditional conceptualization of depression in older adults in this sample. An attempt to improve model fit with the addition of a theoretically related indicator (i.e., worry) failed. The second attempt to improve model fit by incorporating a statistically 
related variable (i.e., apprehension and vigilance) proved more successful. Nevertheless, the combination of positive affect, negative affect, depression, and apprehension and vigilance as indicators for depression does not align with prior research on the structural nature of depression and anxiety in older adults (Cook et al., 2004; Teachman et al., 2007). Instead, the measurement model was more consistent with psychological distress and the alternative hypothesis that depression, anxiety, and agitation were subsumed under an overarching construct of general distress.

It was expected that measures of anxiety other than worry and physiological arousal would correlate with measures of negative affect and agitation such that anxiety and agitation would share physical behaviors represented in both constructs. Marked correlations were found among most of the total and subscale scores for the measures of anxiety and agitation. The anxiety measure, overall, and its subscales of worry, apprehension and vigilance, and motor tension significantly and positively correlated with negative affect, the agitation measure, and the agitation subscales. The anxiety measure subscale of physiological arousal did not correlate with negative affect or agitation, which is consistent with past research on the relationship between anxiety and agitation in older adults with dementia (Twelftree \& Qazi, 2006). However, the worry subscale did significantly correlate with verbal agitation, suggesting anxiety and agitation shared more than physical behaviors. Accordingly, a measurement model for anxiety was specified with negative affect and all indicators of anxiety and agitation specified to load onto it. In contrast to the measurement model for depression, the measurement model for anxiety demonstrated good fit, indicating this model was indicative of anxiety in long-term care residents with dementia. 
Though several significant correlations existed between measures of anxiety and agitation, agitation did not significantly correlate with measures affect, depression, and the anxiety measure subscale of physiological arousal. The lack of significant associations between the measure of agitation and those of affect and depression appear to be contrary to previous findings. Research demonstrates a significant positive relationship between agitation and negative affect, as well as a converse relationship between agitation and positive affect (Cohen-Mansfield, Dakheel-Ali, Jensen, Marx, \& Thein, 2012). Furthermore, negative affect has been found to be predictive of aggressive agitation, whereas positive affect has been shown to predict non-aggressive agitation (Beck et al., 2011). Also, depression and depressive symptoms have been shown to precipitate agitation in older adults with dementia (Kong, 2005). In accordance with the correlations, a measurement model of agitation was specified. Nonetheless, the results of the model indicated good fit, demonstrating this model was suggestive of agitation in this population of long-term care residents with dementia.

The measurement models completed in anticipation of the confirmatory factor analyses were discouraging for estimating a three factor model of depression, anxiety, and agitation. While the models for anxiety and agitation demonstrated good fit, the model for depression was inadmissible. The results of the measurement model analyses indicated the hypothesized three factor model neither represented the theoretical, nor the empirical relationships among the variables for the current sample (Cook et al., 2004; Mineka et al., 1998; Teachman et al., 2007). Given the well-documented relationships among depression, anxiety, and agitation, the alternative model estimating general 
distress as has been demonstrated in a community-dwelling sample of older adults appeared to be the most viable solution (Meeks et al., 2002).

The single factor solution. Through confirmatory factor analyses, this study sought to test two models of the structural relationship of depression, anxiety, and agitation in long-term care residents with dementia. The first model suggested depression and anxiety were linked according to the tripartite model as had been found in younger adult and community-dwelling and outpatient populations of older adults (Cook et al., 2004; Mineka et al., 1998; Teachman et al., 2007) and that agitation and anxiety were connected by shared behaviors. The alternative model indicated depression, anxiety, and agitation were subsumed under construct representing general distress that has been demonstrated in community-dwelling older adults without dementia (Meeks et al., 2002). The main hypothesis that anxiety, depression, and agitation represented separate, but related constructs in long-term care residents with dementia was not supported. Rather, the alternative hypothesis that anxiety, depression, and agitation contributed to an overarching construct of general distress was supported for both the resident and CNA models. The results of this study suggest depression and anxiety may not be differentiated in the same way as is typically seen in younger adult and nondemented, community-dwelling and outpatient psychiatric older adult populations. The addition of agitation to this model did not increase the understanding of the relationships among these constructs. Agitation, as with depression and anxiety, loaded onto the alternative model of general distress, suggesting that differentiating agitation for other indicators of affective distress (i.e., anxiety) was not possible in this sample. 


\section{Considerations for the alternative model of depression, anxiety, and agitation}

in long-term care residents with dementia. This study suggests that, for individuals with dementia, depression, anxiety, and agitation may be best thought of as expressions of general distress rather than being discrete manifestations of psychopathology. This view is consistent with the notion of "behavioral and psychological symptoms of dementia" (BPSD). BPSD has been described as a cluster of emotional, behavioral, cognitive, and neurological symptoms affecting the majority of individuals with dementia that can lead to increased impairment, distress, and mortality (Cerejeira, Lagarto, \& Mukaetova-Ladinska, 2012; Russ, Hamer, Stamatakis, Starr, \& Batty, 2011). BPSD occurs among individuals with a range of cognitive impairment and is most frequent among long-term care residents (Monastero, Mangialsche, Carmarda, Ercolani, \& Carmarda, 2009; Ryu, Ha, Park, Yu, \& Livingston, 2011).

BPSD researchers have described it as a heterogeneous collection of symptoms that subsumes depression, anxiety, and agitation, as well as other related symptoms and sub-syndromes (e.g., aberrant motor behavior, elation, irritability, apathy, disinhibition, delusions, hallucinations, and changes in sleep and appetite); the most prevalent of which include apathy, depression, irritability, agitation, and anxiety (Ryu et al., 2011). Cerejeira and colleagues (2012) note BPSD affects the emotional, cognitive, and behavioral functioning of older adults with dementia and characterize the psychological component of BPSD as being marked by "anhedonia, expression of somatic concerns and anxiety, a subjective experience of fear manifested as apprehension, tension, panic, or worry associated with autonomic activation and observable physical and motor 
manifestations of tension" that varies within the individual over the course of the disease (no page \#). Though BPSD represents a heterogeneous cluster of symptoms that can be difficult to characterize into more specific sub-syndromes, three primary sub-syndromes have emerged: psychosis (hallucinations and delusions), affective (depression and anxiety), and agitation (agitation and irritability) that have been found to remain relatively stable over time in long-term care residents (Dechamps, Jutland, Onifade, Richard-Hartson, \& Bourdel-Marchasson, 2008; Selbæk \& Engedal, 2012). Still, others describe greater overlap in the symptoms included in these sub-syndromes (Mirakhur, Craig, Hart, Mcllroy, \& Passmore, 2004), which is more consistent with our findings.

The results of the confirmatory factor analyses suggest the experience of depression, anxiety, and agitation in long-term care residents with dementia represents a set of interrelated symptoms that may be associated with the onset and progression of dementia. Though not clearly defined as disorders, the experience of depression, anxiety, and agitation warrant clinical attention in this population. Cerejeira et al. (2012) recommend identifying the nature of the symptoms or sub-syndromes through accurate assessment of older adults' personal experiences with dementia, such that treatment may be tailored to their specific needs. However, the authors also point out this may be difficult in such a heterogeneous syndrome.

\section{The Relationship between Anxiety and Agitation}

The hypothesis that global cognitive functioning would moderate the relationship between anxiety and agitation in older adults with dementia living in long-term care was not supported. Consistent with bivariate correlations reported earlier, a significant main 
effect of anxiety on agitation was found, such that older adults experiencing clinically significant anxiety (RAID score greater than or equal to 11) experienced increased frequency of agitated behavior regardless of level of cognitive functioning. This result was found for both resident and CNA responses to the RAID. However, consistency between results for the resident and CNA responses to the RAID must be interpreted cautiously as the CNA data violated the assumption of homogeneity of variance. Past research highlights the co-occurrence of anxiety and agitation in older adults with dementia, but also demonstrates inconsistencies in the findings on the association between anxiety and cognitive functioning. This study replicates past research on the common co-occurrence of anxiety and agitation (Cohen-Mansfield \& Billig, 1986; Ferretti et al., 2001; Mega et al., 1996; Ownby et al., 2000; Porter et al., 2003). The findings from this study imply some elements of anxiety may facilitate agitation, but that anxiety is not the sole reason for agitation, which has also been suggested by previous research (Twelftree \& Qazi, 2006). Consequently, it is important to consider anxiety as a possible source for agitation, but to also assess for other possible causes of agitation (Kong, 2005).

No association was found between level of cognitive functioning and anxiety or agitation, furthering past findings on a lack of an association between degree of cognitive impairment and severity of anxiety (Chemerinski et al., 1998; Forsell \& Winblad, 1997; Orrell \& Bebbington, 1996). The relationship between current anxiety and agitation appears to arise at any point in the dementia process. Neither anxiety, nor agitation were significantly associated with global cognitive functioning, disconfirming the assertion that agitation is the behavioral manifestation of anxiety as dementia progresses (Mintzer 
\& Brawman-Mintzer, 1996). Anxiety appears to persist across the course of dementia, rather than symptoms increasing or decreasing as dementia progresses. Symptoms of anxiety that overlap with agitation may persist throughout the dementia process perhaps leading to expression of these shared symptoms and putting long-term care residents with dementia at greater risk for experiencing agitation generally (Krasucki, Howard, \& Mann, 1998). Clinically, screening, assessment, and treatment of anxiety may reduce agitated behaviors in long-term care residents with dementia. However, as noted above, providers should be wary of other sources of agitation and treat accordingly.

\section{The Role of Mental Health History in the Experience of Current Anxiety}

The final hypothesis that global cognitive functioning would moderate the relationship between mental health history and current experience of anxiety in long-term care residents with dementia was not supported. However, a significant main effect of mental health history on current anxiety was found. This result was consistently found for general mental health, history of mood or anxiety disorder, history of depression, and history of anxiety for resident ratings of anxiety. The more specific the history question, the larger the effect, such that a history of anxiety disorder had the strongest relationship with anxiety, whereas a general history of mental health treatment had the weakest.

The main effect of mental health history on current anxiety was less consistent

for CNA responses to the RAID. A significant main effect of history of either mood or anxiety disorder on current anxiety, as well as a significant main effect of history of anxiety on current anxiety was found; but not significant main effects for general mental health history or history of depression alone. Notably, the main effect of history of anxiety on current anxiety was greater than the main effect of history of mood or anxiety 
disorder on current anxiety, such that long-term care residents with dementia who had a history of anxiety experience greater severity of anxiety symptoms as rated by their CNAs.

Many older adults report having had anxiety "all their lives" (p. 26; Roux et al., 2005). A bimodal distribution of the onset of anxiety exists with many older adults reporting onset before the age of 50 (Roux et al., 2005). Current anxiety symptoms and disorders are prevalent among long-term care residents and older adults with dementia (Ballard et al., 1996; Ballard et al., 1994; Chemerinski et al., 1998; Ferretti et al., 2001; Neville \& Teri, 2011; Smallbrugge, Pot, et al., 2005; Trachtenberg et al., 2002). Current anxiety symptoms and disorders frequently co-occur with depressive symptoms and disorders among these populations with the presence or history of either disorder leading to increased severity of the other symptoms or disorder (Smallbrugge, Jongenelis, et al., 2005; Ballard et al., 1994; Ferretti et al., 2001; Gibbons et al., 200; Harwood et al., 1998; Mack et al., 1999; Mega et al., 1996; Tariot et al., 1995; Teri et al, 1999; Teri et al, 1992). Evidence from community-dwelling older adults and those living in long-term care suggests older adults with dementia may be able to experience current anxiety. However, it has been hypothesized that changes in cognitive functioning may limit their ability to experience anxiety. Results from the research on the experience of anxiety have been inconsistent with studies noting an increase in anxiety as dementia progresses, while others have found anxiety peaks when older adults with dementia begin to experience more moderate global cognitive impairment and still others suggesting greater anxiety is associated with mildly impaired global cognitive functioning (Chemerinski et al., 1998; 
Forsell \& Winblad, 1997; Orrell \& Bebbington, 1996; Porter et al., 2003; Teri et al., 1999; Starkstein et al., 1997). Contradictions in the research on anxiety and cognitive impairment highlighted the need to identify other predictors of anxiety in older adults with dementia, including pre-existing mental health disorders (Schoevers et al., 2003).

The current research demonstrates a lack of association between anxiety and global cognitive functioning; but a strong association between pre-existing mental health disorder, particularly history of an anxiety disorder, and current anxiety. The results of this study support the maintenance of anxiety into older adulthood among long-term care residents regardless of cognitive functioning. Moreover, they suggest changes in cognitive functioning do not affect older adults' ability to experience and report anxiety, providing support for Cohen (1998) and James (1999)'s contention that older adults with dementia are fully capable of feeling and expressing anxiety. At the same time, Cohen and James contend that anxiety in older adults with more advanced dementia is manifested as "catastrophic reactions" and "dysfunctional behaviors and actions" frequently misattributed to agitation (pp. 106 and 347, respectively). The results of this study, however, indicate that clinically significant anxiety is related to increased agitation apart from global cognitive functioning; suggesting older adults with severe cognitive impairment are capable of expressing anxiety in a manner other than aberrant behaviors. As discussed above, anxiety may be a source for agitation, but other reasons for agitation should be considered in conjunction with the experience of anxiety. As with the relationship between current anxiety and increased agitation, the relationship between mental health history (especially history of anxiety) and current anxiety in long-term care residents with dementia points to the need to screen for anxiety in this population to 
provide optimal care in light of the persistence of anxiety across the lifespan.

Additionally, although informant observation of anxiety is valuable, consideration of older adult report is imperative to screening for anxiety even among long-term care residents with severe cognitive impairment.

\section{Limitations}

Sample size. Conducting research with long-term care facilities presents challenges to collecting optimal sample sizes for adequate power for data analyses. Several barriers were encountered when attempting to recruit participants for the present study from systemic challenges to resident and proxy refusal. Many long-term care facilities declined participation in the study due to constraints on site liaison work time or lack of interest - reasons often stated by facilities for lack of participation in dementia research (Goodman et al., 2011). Once facilities had been recruited, some site liaisons struggled to identify residents who met eligibility criteria, citing residents' inability to communicate orally with researchers. At an individual level, residents most often refused to participate in the study due to lack of interest. When responsible parties were contacted for informed consent by proxy, several requested that the resident not participate out of concern that participation would create distress. In spite of these challenges, this study achieved over a one-third response rate. Nonetheless, recruitment challenges prevented the proposed sample size from being obtained. Consequently, the study is under-powered for the proposed analyses. Therefore, conclusions drawn from the results of this study may not be consistent with a larger, more representative sample of the target population. Recruitment processes, and ultimately sample size, may have 
been improved through increased express communication with older adults and their formal caregivers and responsible parties about the purpose and process of the research and in-person recruitment of nursing homes (Elkins et al., 2011; Thompson, Heller, \& Rody, 1994).

Homogeneity of the sample. Overall, the sample recruited for the present study was representative of the national long-term care population. Yet, limitations in the number of racial and ethnic minorities recruited for this study may restrict generalizability to these populations. For instance, Latino older adults are one of the fastest growing ethnic minority age cohorts with an increasing number of Latino older adults entering nursing homes (Gallagher-Thompson, Solano, \& Areán, 2002; Jones et al., 2009). The findings from this study may not be applicable to ethnic minorities given social and cultural differences between European American and Latino, as well as African American, older adults. Regional restrictions may have inhibited the recruitment of racial and ethnic minorities into this study. There is a paucity of research on racial and ethnic minority older adults with anxiety, though attempts to understand prevalence and presentation have expanded in the past decade (Brenes et al., 2008; Cohen, Magai, Yaffe, \& Walcott-Brown, 2006; Ford et al., 2007; Mehta et al., 2003; Ostir \& Goodwin, 2006). Given the limited number of racial and ethnic minorities who participated in this sample, the results may not generalize beyond European American long-term care residents with dementia. Additional recruitment of racial and ethnic minorities into this study may have furthered knowledge of anxiety among these populations. Recruitment of racial and ethnic minorities may have been enhanced by more openly addressing issues of distrust of the research process and stigma associated with mental illness, increasing awareness of 
normal cognitive aging versus dementia, and developing culturally appropriate recruitment materials (Areán \& Gallagher-Thompson, 1996; Danner, Darnell, \& McGuire, 2011; Gallagher-Thompson et al., 2002).

Measures. Two measures were selected for this study to decrease burden on potential participants - the PHQ-9 and the BIMS. The PHQ-9 is a measure of depression widely used in community and hospital settings. It has been evaluated with older adult, home health, and rehabilitation populations (Saliba \& Jones, 2008). Until testing was completed with a large long-term care sample, the psychometric properties of the PHQ-9 when used with the long-term care population had not been established. Saliba and Jones (2008) reported that inter-rater reliability was excellent and the validity was moderate across a range of cognitive functioning. In practice, this measure may be completed by nursing staff designated to complete MDS measures with residents who are less familiar with residents' care. This may result in underestimations of depressive symptoms (Phillips, 2012). Subsequent research has brought into question the convergent validity of the PHQ-9 with long-term care residents with dementia as this measure was not shown to correlate with depression diagnoses as indicated in medical charts (Phillips, 2012). Other measures, such as the CSDD, that have been more extensively studied in the longterm care population may have been better indicators of depression this sample (Kurlowicz, Evans, Strumpf, \& Maislin, 2002; Leontjevas, van Hooren, \& Mulders, 2009; Phillips, 2012). Granted unconstrained contact with participants, another wellestablished measure of depression in older adults with dementia may have yielded results consistent with prior research on the tripartite model of depression and anxiety in community-based and psychiatric outpatient populations of older adults (Cook et al., 
2004; Teachman et al., 2007). Practically, selecting this measure of depression optimized time engaged in data collection with a medically frail population and, in due course, bolstered sample size.

The BIMS is brief measure of global cognitive functioning originally developed in Veterans Affairs Hospital extended care facilities (Chodosh, 2008). Results from the studies conducted with veterans, indicated excellent convergent and criterion validity with other measures frequently used to screen for cognitive impairment in long-term care and improved psychometric properties over the cognitive measure used in the prior version of the MDS (Chodosh, 2008). Subsequent research involving 71 community long-term care facilities with a population similar to the sample collected for the present study replicated these findings (Saliba \& Buchanan, 2008). Several shortcomings of the BIMS have been noted. First, the BIMS lacks a test of executive functioning that may miss some impaired residents. Second, the BIMS was tested against the standard of the Modified Mini-Mental Status Exam and not against other criterion standards, namely a formal neuropsychological evaluation, limiting the diagnostic utility of this measure. No data has been reported on the association between the BIMS and diagnoses of dementia in long-term care residents (Chodosh et al., 2008). Other measures that have established use with older adults with dementia, such as the Montreal Cognitive Assessment (MoCA), may have yielded different results if used in this study. The MoCA has demonstrated improved sensitivity and specificity in screening for cognitive impairment regardless of etiology of dementia over other measures previously routinely used in longterm care (i.e., the Mini Mental State Examination; Pendlebury et al., 2012). Differences in sensitivity may have led to altered findings in regard to the moderating function of 
global cognitive impairment and the relationships between current anxiety and agitation, as well as the relationship between mental health history and current anxiety.

The RAID is relatively new measure of anxiety specifically designed for the assessment of anxiety in older adults with dementia. The psychometric properties of the RAID have been demonstrated in a handful of studies using samples of mainly community-dwelling older adults with dementia. It has been touted as a quick and userfriendly measure of anxiety in older adults with dementia (Shankar et al., 1999). The psychometric properties of the RAID have been established in samples of communitydwelling older adults, demonstrating very good internal consistency and moderate correlations with other measures of anxiety shown to be reliable and valid in older adult populations, as well as sufficient discriminant validity (Kabacoff, Segal, Hersen, \& Van Hasselt, 1997; Shankar et al., 1999; Snow et al., 2012). The advantage of the RAID over other measures remains dubious, given comparable psychometric properties of measures like the PSWQ and the Beck Anxiety Inventory (BAI) when screening for anxiety in older adults (Beck, Stanley, \& Zebb, 1995; Cook et al., 2004; Kabacoff et al., 1997). Measures specifically designed to assess for worry may be more appropriate since subjective symptoms of anxiety and GAD are most prevalent among older adults with dementia (Ballard et al., 1996; Ballard et al., 1994; Chemerinski et al., 1998; Ferretti et al., 2001; Mack et al, 1999). However, employing measures of worry may confound the relationship between depression and anxiety in this population, making distinguishing these constructs more difficult. Though the BAI may be valid for the use with community-dwelling older adults, the use of this measure with long-term care residents may be complicated by medical co-morbidities frequent in this population. 
Further research may be needed on the psychometric properties of the RAID when used with long-term care residents with dementia, and appropriate measures of anxiety, generally.

Restricted range of measures. Several of the measures used in this study demonstrated restricted range, including the RAID (both resident and CNA) and the CMAI. Scores for the RAID range from 0 to 54 . In this study, scores ranged from 0 to 37 with the majority of participants not attaining the clinical cut score of 11 . Past research has demonstrated similar results in an inpatient and day hospital sample of older adults with dementia (range $=0-39 ; N=83,62.0 \%$ female; $M$ age $=79.1, S D=7.0$ ). Scores for the CMAI range from 0-203; however, in this study, the score ranged from 0 33 with $61.5 \%$ not meeting criteria for presence of agitation. Research with other populations of older adults with dementia has demonstrated a broader range of scores with this measure. For instance, Weiner and colleagues (2000) found a range from 2-122 in a community dwelling population of older adults with dementia $(N=148 ; 55.0 \%$ female; $M$ age $=74.8, S D=7.1)$. Still, in a sample of nursing home residents $(N=408$, $77.45 \%$ female, $M$ age $=85$ ), Cohen-Mansfield, Marx, and Rosenthal (1989) found more restricted ranges of scores across day (range $=0-23, M=3.8, S D=4.0$ ), evening (range = $0-22, M=3.3, S D=3.8$ ), and night shifts (range $=0-16, M=2.1, S D=2.8$ ). Restricted ranges in measures limits the ability to confidently make conclusions about the significant correlations found in the data and to generalize findings beyond the range and sample in which they were found (Warner, 2013). Restricted ranges may over- or underestimate (as in the case of extreme groups) the size of the correlation. Ideally, larger samples representative of the population would avoid this issue. In the case of 
research with long-term care residents with dementia, obtaining large, representative samples may be challenging. Those who agree to participate may represent a select sample of altruistic older adults. Nevertheless, reports of ranges for the RAID and CMAI in prior research suggest conclusions may be appropriately drawn from the results found in this study.

Diagnosis of dementia. Long-term care residents were eligible to participate in the current study if a dementia diagnosis was indicated in their medical charts. The validity and reliability of the dementia diagnoses, however, are questionable as the source and reasoning of the diagnosis are not regularly documented in the chart. Diagnoses listed in the MDS 3.0 are coded as active if the diagnosis has been documented in the last 60 days and has a relationship to functional, cognitive, mood, or behavior status or medical treatments, nursing monitoring, or risk of death. Evidence of active diagnoses may be found in medical providers' notes, medication sheets, doctors' orders, and consults and official diagnostic reports among other sources of information. Thus, a long-term care resident with an active diagnosis of dementia need not have a formal neuropsychological evaluation that includes a dementia diagnosis, making the accuracy of the dementia diagnosis uncertain. Likewise, there is a dearth of research on the criterion validity of the cognitive measure in the MDS 3.0 and its ability to predict and detect dementia, suggesting that use of this measure as an indication of an active dementia diagnosis may be misleading. With the lack of certainty on the source and nature of the dementia diagnosis, the results of this study may not be generalizable to all long-term care residents with dementia. The results of this study might have been 
improved by including residents who have received dementia diagnoses independent from long-term care medical charting.

\section{Future Directions}

Although arduous efforts were made to recruit a large, representative sample of long-term care residents with dementia, barriers in the recruitment process limited the sample size for this study. Future research involving long-term care residents with dementia should further employ "best practices" for recruiting this population, including in-person recruitment with nursing home administrators and other staff and psychoeducation on the research process. These recruitment strategies may be extended to obtain a more racially and ethnically diverse sample of residents by providing additional education on dementia and developing recruitment materials tailored to these populations. Augmented efforts in recruitment may lead to increased participation, a larger and more representative sample, and greater generalizability to other nursing home populations outside of the region used for this study.

The measures selected to complete this study may have presented challenges, specifically the PHQ-9, BIMS, and RAID. Although these measures were selected based on sound empirical support for use with older adults with dementia, they are relatively understudied. Other measures of depression, global cognitive functioning, and anxiety, such as the CSDD, MoCA, and PSWQ, may have yielded results more consistent with those that were hypothesized. These measures may be more sensitive to certain aspects of mental health and dementia and have more clinical utility with the target population. Additional research is needed on the psychometric properties of the PHQ-9, BIMS, and RAID when used with long-term care residents with dementia. Subsequent research 
should consider use of more established measures when attempting to explore the relationships among depression, anxiety, and dementia in older adults.

Due to time constraints associated with completing this research, residents with a diagnosis of dementia as listed in their medical charts were deemed eligible to participate in this study. Because the accuracy of the dementia diagnoses was questionable, the results of this study may not be generalizable to all long-term care residents with dementia. Given adequate time, future research on anxiety in long-term care residents should consider completing independent neuropsychological evaluations on participants. Not only would this increase the generalizability, but with sufficient sample size, may create the opportunity to differentiate the experience of anxiety in older adults with varied dementia diagnoses.

\section{Final Conclusions}

In sum, this study examined the relationships among anxiety, depression, and agitation in older adults with dementia residing in long-term care, as well as the roles of global cognitive functioning and mental health history in the experience of anxiety in this population. The primary hypothesis that anxiety, depression, and agitation represented separate, but related constructs in long-term care residents with dementia was not supported. Rather, the alternative hypothesis that these constructs were included in an overarching construct of general distress was supported. In addition, it was hypothesized that global cognitive functioning would moderate the relationships between experience of current anxiety and agitation and between mental health history and current anxiety. Global cognitive functioning moderated neither the relationship between current anxiety and agitation, nor between mental health history and current anxiety. The results of this 
study may have been affected by the sample size, measures selected, and definition of the eligibility criterion of dementia diagnosis. Obtaining a larger, more representative sample size; modification in selection of measures of depression, global cognitive functioning, and anxiety; and explication of dementia diagnosis may clarify the relationships among depression, anxiety, and agitation in long-term care residents with dementia.

Furthermore, these alterations in the research process may clarify the role of global cognitive functioning in the association between anxiety and agitation and between mental health history and anxiety.

To improve care for residents with dementia, thorough evaluations inclusive of mental health history should be conducted regularly. Assessment may elucidate the older adults' experience with dementia, including concurrent depression and anxiety symptoms and disorders. With this information in hand, providers may be able to ascertain possible etiologies or precipitants of agitated behavior and provide targeted interventions. Moreover, when addressing agitated behaviors in long-term care residents with dementia, providers should be mindful of affective origins of the agitation, especially when older adults' have a history of mental health disorder. 


\section{REFERENCES}

Algase, D. L., Beck, C., Kolanowski, A., Whall, A., Berent, S. K., Richards, K., et al. (1996). Need-driven dementia-compromised behavior: An alternative view of disruptive behavior. American Journal of Alzheimer's Disease and Other Dementias, 11, 10-19.

Allen, R.S., Burgio, L.D., Roth, D.L., Ragsdale, R., Gerstle, J., Bourgeois, M.S. (2003). The Revised Memory and Behavior Problems Checklist - Nursing Home: Instrument development and measures of burden among certified nursing assistants. Psychology and Aging, 18, 886-895.

Areán, P.A., \& Gallagher-Thompson, D. (1996). Issues and recommendations for the recruitment and retention of older ethnic minority adults into clinical research. Journal of Consulting and Clinical Psychology, 64, 875-880.

Ashcraft, A.S., Owen, D.C., \& Feng, P. (2006). A comparison of cognitive and functional care differences in four long-term care settings. Journal of American Medical Directors Association, 7, 96-101.

Ballard, C., Boyle, A., Bowler, C., \& Lindesay, J. (1996). Anxiety disorders in dementia sufferers. International Journal of Geriatric Psychiatry, 11, 987-990.

Ballard, C.G., Mohan, R.N.C., Patel, A., \& Graham, C. (1994). Anxiety disorder in dementia. Irish Journal of Psychological Medicine, 11, 108-109. 
Barlow, D.H. (2002). Anxiety and its disorders: The nature and treatment of anxiety and panic. New York: The Guilford Press.

Beck, A. T., Emery, G., \& Greenberg, R.L. (1985). Anxiety disorders and phobias: A cognitive perspective. New York: Basic Books, Inc.

Beck, J.G., Stanley, M., \& Zebb, B.J. (1996). Characteristics of Generalized Anxiety Disorder in older adults: A descriptive study. Behaviour Research and Therapy, $34,225-234$.

Beck, J.G., Stanley, M.A., \& Zebb, B.J. (1995). Psychometric properties of the Penn State Worry Questionnaire in older adults. Journal of Clinical Geropsychology, $1(1), 33-42$.

Bentler, P.M. (1990). Comparative fit indexes in structural models. Psychological Bulletin, 107, 238-246.

Bögels, S.M., \& Brechman-Toussaint, M.L. (2006). Family issues in child anxiety: Attachment, family functioning, parent rearing and beliefs. Clinical Psychology Review, 26, 834-856.

Bollen, K.A. (1989). Structural equations with latent variables. New York: Wiley.

Brenes, G.A., Guralnik, J.M., Williamson, J., Fried, L.P., \& Penninx, B.W.J.H. (2005). Correlates of anxiety symptoms in physically disabled older women. American Journal of Geriatric Psychiatry, 13, 15-22.

Brenes, G.A., Knudson, M., McCall, W.V., Williamson, J.D., Miller, M.E., \& Stanley, M.A. (2008). Age and racial differences in the presentation and treatment of Generalized Anxiety Disorder in primary care. Journal of Anxiety Disorders, 22, $1128-1136$. 
Buckles, V.D., Powlishta, K.K., Palmer, J.L., Coats, M., Hosto, T., Buckley, A., \& Morris, J.C. (2003). Understanding of informed consent by demented individuals. Neurology, 61, 1662-1666.

Carstensen, L.L., \& Turk-Charles, S. (1994). The salience of emotion across the adult life span. Psychology and Aging, 9, 259-264.

Cerejeira, J., Lagarto, L., \& Mukaetova-Ladinska, E.B. (2011). Behavioral and psychological symptoms of dementia. Frontiers in Neurology, 3, 73.

Chemerinski, E., Petracca, G., Manes, F., Leiguarda, R., \& Starkstein, S.E. (1998). Prevalence and correlates of anxiety in Alzheimer's disease. Depression and Anxiety, 7, 166-170.

Chodosh, J., Edelen, M.O., Buchanan, J.L., Yosef, J.A., Ouslander, J.G., Berlowitz, D.R.,...Saliba, D. (2008). Nursing home assessment of cognitive impairment: Development and testing of a brief instrument of mental status. Journal of the American Geriatrics Society, 56, 2069-2075.

Chou, K. (2009). Age at onset of Generalized Anxiety Disorder in Older Adults. American Journal of Geriatric Psychiatry, 17, 455-464.

Chorpita, B.F., \& Barlow, D.H. (1998). The developmental of anxiety: The role of control in the environment. Psychological Bulletin, 124, 3-21.

Clark, L.A., Watson, D., \& Mineka, S. (1994). Temperament, personality, and the mood and anxiety disorders. Journal of Abnormal Psychology, 103, 103-116.

Cohen, G. (1998). Anxiety in Alzheimer's disease: Theoretical and clinical perspectives. Journal of Geriatric Psychiatry, 31, 103-115. 
Cohen, C.I., Magai, C., Yaffee, R., \& Walcott-Brown, L. (2006). The prevalence of anxiety and associated factors in a multiracial sample of older adults. Psychiatric Services, 57, 1719-1725.

Cohen-Mansfield, J. (1986). Agitated behaviors in the elderly II. Preliminary results in the cognitive deteriorated. Journal of the American Geriatrics Society, 34, 722727.

Cohen-Mansfield, J. (2008). Agitated behaviors in persons with dementia: The relationship between type of behavior, its frequency, and its disruptiveness. Journals of Psychiatric Research, 43, 64-69.

Cohen-Mansfield, J., \& Billig, N. (1986). Agitated behaviors in the elderly I. A conceptual review. Journal of the American Geriatrics Society, 34, 711-721.

Cohen-Mansfield, J., \& Libin, A. (2004). Assessment of agitation in elderly patients with dementia: Correlations between informant rating and direct observation. International Journal of Geriatric Psychiatry, 19, 881-891.

Cohen-Mansfield, J., Dakheel-Ali, M., Jensen, B., Marx, M.S., \& Thein, K. (2012). An analysis of the relationships among engagement, agitated behavior, and affect in nursing home residents with dementia. International Psychogeriatrics, 24, 742752.

Cohen-Mansfield, J., Marx, M., \& Rosenthal, A. (1989). A description of agitation in a nursing home. Journal of Gerontology, 44, 77-84.

Cohen-Mansfield, J., Werner, P., Watson, V., \& Pasis, S. (1995). Agitation among elderly persons at adult-day centers: The experiences of relatives and staff members. International Psychogeriatrics, 7, 447-485. 
Cook, J.M., Orvaschel, H., Simco, E., Hersen, M., \& Joiner, T. (2004). A test of the tripartite model of depression and anxiety in older adult psychiatric outpatients. Psychology and Aging, 19, 444-451.

Dalrymple, K.L., \& Zimmerman, M. (2008). Screening for social fears and social anxiety disorder in psychiatric outpatients. Comprehensive Psychiatry, 49, 399-406.

Danner, D.D., Darnell, K.R., \& McGuire, C. (2011). African American participation in Alzheimer's disease research that includes brain donation. American Journal of Alzheimer's Disease and Older Dementias, 26, 469-476.

Dechamps, A., Jutland, M.A., Onifade, C., Richard-Hartson, S., \& Bourdel-Marchasson, I. (2008). Co-occurrence of neuropsychiatric symptoms in demented and psychotic institutionalized elderly. International Journal of Geriatric Psychiatry, 23, 1182-1190.

Deer, T.M., \& Calamari, M.J. (1998). Panic symptomatology and anxiety sensitivity in older adults. Journal of Behavior Therapy and Experimental Psychiatry, 29, 303316.

Depp, C., Woodruff-Borden, J., Meeks, S., Gretarsdottir, E., \& DeKryger, N. (2005). The phenomenology of non-clinical panic in older adults in comparison to younger adults. Journal of Anxiety Disorders, 19, 503-519.

Eley, T.C. (2001). Contributions of behavioral genetics research: Quantifying genetic, shared environmental and nonshared environmental influences. In M.W. Vasey \& M.R. Dadds (Eds), The development psychopathology of anxiety (pp. 45-59). New York, NY: Oxford University Press. 
Ferretti, L., McCurry, S.M., Logsdon, R., Gibbons, L., \& Teri, L. (2001). Anxiety and Alzheimer's disease. Journal of Geriatric Psychiatry and Neurology, 14, 52-57. Field, A. (2005). Discovering statistics using SPSS ( $2^{\text {nd }}$ ed.). London: Sage Publications. Finkel, S.I., Lyons, J.S., \& Anderson, R.L. (1992). Reliability and validity of the CohenMansfield Agitation Inventory in institutionalized elderly. International Journal of Geriatric Psychiatry, 7, 487-490.

First, M.B., Gibbon, M., Spitzer, R.L., \& Williams, J.B.W. (2001). Users guide for the SCID-I: Structured Clinical Interview for DSM-IV-TR axis I disorders (research version). New York, NY: Biometrics Research, New York State Psychiatric Institute.

Flint, A.J. (1994). Epidemiology and comorbidity of anxiety disorders in the elderly. American Journal of Psychiatry, 151, 640-649.

Flint, A.J., Peasely-Miklus, C., Meyers, B.S., Rothschild, A.J., \& Whyte, E.M. (2010). Effect of age on the frequency of anxiety disorders in major depression with psychotic features. American Journal of Geriatric Psychiatry, 18, 404-412.

Ford, B.C., Bullard, K.M., Taylore, R.J., Toler, A.K., Neighbors, H.W., \& Jackson, J.S. (2007). Lifetime and 12-month prevalence of Diagnostic and Statistical Manual of Mental Disorders, Fourth Edition disorder among older African Americans: Findings from the National Survey of American Life. American Journal of Geriatric Psychiatry, 15, 652-659.

Forsell, Y., \& Winblad, B. (1997). Anxiety disorders in non-demented and demented elderly patients: Prevalence and correlates. Journal of Neurology, Neurosurgery, \& Psychiatry, 62, 294-295. 
Fullerton, C.A., McGuire, T.G., Feng, Z., Mor, V., \& Grabowski, D.C. (2009). Trends in mental health admissions to nursing homes, 1999-2005. Psychiatric Services, 60 , 965-971.

Gallagher-Thompson, D., Solano, N., Coon, D., \& Areán, P. (2002). Recruitment and retention of Latino dementia family caregivers in intervention research: Issues to face, lessons learned. The Gerontologist, 43(1), 45-51.

Gibbons, L.E., Teri, L., Logsdon, R.G., \& McCurry, S.M. (2006). Assessment of anxiety in dementia: An investigation into the association of different methods of measurement. Journal of Geriatric Psychiatry and Neurology, 19, 202-208.

Goodman, C., Baron, N.L., Machen, I., Stevenson, E., Evans, C., Davies, S.L., \& Iliffe, S. (2011). Culture, consent, costs and care homes: Enabling older people with dementia to participate in research. Aging and Mental Health, 15, 475-481.

Gretarsdottir, E., Woodruff-Borden, J., Meeks, S., \& Depp, C.A. (2004). Social anxiety in older adults: Phenomenology, prevalence, and measurement. Behaviour Research and Therapy, 42, 459-475.

Gum, A.M., King-Kallimanis, B., \& Kohn, R. (2009). Prevalence of mood, anxiety, and substance-abuse disorders for older Americans in the National Comorbidity Survey-Replication. American Journal of Geriatric Psychiatry, 17, 769-781.

Harwood, D.G., Ownby, R.L., Barker, W.W., \& Duara, R. (1998). The behavioral pathology in Alzheimer's disease scale (BEHAVE-AD): Factor structure among community-dwelling Alzheimer's disease patients. International Journal of Geriatric Psychiatry, 13, 793-800. 
He, W., Sengupta, M., Velkoff, V.A., DeBarros, K.A. (2005). 65+ in the United States: 2005. Retrieved from http://www.census.gov/prod/2006pubs/p23-209.pdf

Horn, S.D., Buerhaus, P., Bergstrom, N., \& Smout, R.J. (2005). RN staffing time and outcomes of long-stay nursing home residents. American Journal of Nursing, 105, $58-70$.

Hu, L., \& Bentler, P.M. (1999). Cut off criteria for fit indices in covariance structure analysis: Conventional criteria versus new alternative. Structural Equation Modeling, 6(1), 1-55.

Hudon, C., Voyer, P., Tremblay, I., Tardif, S., \& Carmichael, P.H. (2010). Differentiation of the pattern of cognitive impairment between depression and non-depressed patients with dementia living in long-term care facilities. Aging and Mental Health, 14, 293-302.

James, I.A. (1999). Using a cognitive rationale to conceptualize anxiety in people with dementia. Behavioural and Cognitive Psychotherapy, 27, 345-351.

Jones, A.L., Dwyer, L.L., Bercovitz, A.R., \& Strahan, G.W. (2009). The National Nursing Home Survey: 2004 overview. National Center for Health Statistics. Vital and Health Statistics, 13 (167).

Kabacoff, R.I., Segal, D.L., Hersen, M., \& Van Hasselt., V.B. (1997). Psychometric properties and diagnostic utility of the Beck Anxiety Inventory and the State-Trait Anxiety Inventory with older adult psychiatric outpatients. Journal of Anxiety Disorders, 11, 33-47.

Kong, E. (2005). Agitation in dementia: Concept clarification. Nursing Theory and Concept Development or Analysis, 52, 526-536. 
Krasucki, C., Howard, R., \& Mann, A. (1998). The relationship between anxiety disorders and age. International Journal of Geriatric Psychiatry, 13, 79-99.

Labouvie-Vief, G. (2008). When differentiation and negative affect lead to integration and groth. American Psychologist, 63, 564-565.

Lawton, M.P., Van Haitsma, K., \& Klapper, J. (1996). Observed affect in nursing home resident's with Alzheimer's disease. Journals of Gerontology. Series B, 51, 3-14.

Lenze, E.J., Mulsant, B.H., Shear, M.K., Schulberg, H.C., Dew, M.A., Begley, A.E., et al. (2000). Comorbid anxiety disorders in depressed elderly patients. American Journal of Psychiatry, 157, 722-728.

Lenze, E.J., \& Wetherell, J.L. (2011). A lifespan view of anxiety disorders. Dialogues in Clinical Neuroscience, 13, 381-399.

Le Roux, H., Gatz, M., \& Wetherell, J.L. (2005). Age at onset of Generalized Anxiety Disorder in older adults. American Journal of Geriatric Psychiatry, 13, 23-30.

Logsdon, R.G., Teri, L., Weiner, M.F., Gibbons, L.E., Raskind, M., Peskind, E., et al. (1999). Assessment of agitation in Alzheimer's disease: The Agitated Behavior in Dementia scale. Journal of the American Geriatrics Society, 47, 1354-1358.

Mack, J.L., Patterson, M.B., \& Tariot, P.N. (1999). Behavior Rating Scale for Dementia: Development of test scales and presentation of data for 555 individuals with Alzheimer's disease. Journal of Geriatric Psychiatry and Neurology, 12, 211223.

Meeks, S., Woodruff-Borden, J., \& Depp, C.A. (2003). Structural differentiation of selfreported depression and anxiety in late life. Journal of Anxiety Disorders, 17, 627-646. 
Mega, M.S., Cummings, J.L., Fiorello, T., \& Gornbein, J. (1996). The spectrum of behavioral changes in Alzheimer's disease. Neurology, 46, 130-135.

Mehta, K.M., Simonsick, E.M., Penninx, B.W., Schulz, R., Rubin, S.M., Satterfield, S., Yaffe, K. (2003). Prevalence and correlated of anxiety symptoms in wellfunctioning older adults: Findings from the health aging and body composition study. Journal of the American Geriatrics Society, 51, 499-504.

Miller, R.J., Snowdon, J., \& Vaughan, R. (1995). The use of the Cohen-Mansfield Agitation Inventory in the assessment of behavioral disorders in nursing homes. Journal of the American Geriatric Society, 43, 546-549.

Mineka, S., Watson, D., \& Clark, L.A. (1998). Comorbidity of anxiety and unipolar mood disorders. Annual Review of Psychology, 49, 377-412.

Mintzer, J.E., \& Brawman-Mintzer, O. (1996). Agitation as possible expression of generalized anxiety disorder in demented elderly patients: Toward a treatment approach. Journal of Clinical Psychiatry, 57 (Suppl. 7), 55-63.

Mirakhur, A., Craig, D., Hart, D.J., Mcllroy, S.P., \& Passmore, A.P. (2004). Behavioural and psychological syndromes in Alzheimer's disease. International Journal of Geriatric Psychiatry, 19, 1035-1039.

Monastero, R., Mangialasche, F., Carmarda, C., Ercolan, S., \& Carmarda, R. (2009). A systemic review of neuropsychiatric symptoms in mild cognitive impairment. Journal of Alzheimer's Disease, 18, 11-30.

Neville, C., \& Teri, L. (2011). Anxiety, anxiety symptoms, and associations among older people with dementia in assisted-living facilities. International Journal of Mental Health Nursing, 20, 195-201. 
Norton, M.J., Allen, R.S., Snow, A.L., Hardin, J.M., \& Burgio, L.D. (2010). Predictors of need-driven behaviors in nursing home residents with dementia and associated certified nursing assistant burden. Aging and Mental Health, 14, 303-309.

Orrell, M., \& Bebbington, P. (1996). Psychosocial stress and anxiety in senile dementia. Journal of Affective Disorders, 39, 165-173.

Ostir, G.V., \& Goodwin, J.S. (2006). High anxiety is associated with an increased risk of death in an older tri-ethnic population. Journal of Clinical Epidemiology, 59, 534540.

Ownby, R.L., Harwood, D.G., Barker, W.W., \& Duara, R. (2000). Predictors of anxiety in patients with Alzheimer's disease. Depression and Anxiety, 11, 38-42.

Pendlebury, S.T., Marwick, A., de Jager, C.A., Zamboni, G., Wilcock, G.K., \& Rothwell, P.M. (2012). Differences in cognitive profile between TIA, stroke and elderly memory research subjects: A comparison of the MMSE and MoCA. Cerebrovascular Diseases, 34(1), 48-54.

Phillips, L.J. (2012). Measuring symptoms of depression: Comparing the Cornell Scale for Depression in Dementia and the Patient Health Questionnaire-9-Observation Version. Research in Gerontological Nursing, 5(1), 34-42.

Porter, V.R., Buxton, W.G., Fairbanks, L.A., Strickland, T., O'Connor, S.M., RosenbergThompson, S., et al. (2003). Frequency and characteristics of anxiety among patients with Alzheimer's disease and related dementias. Journal of Neuropsychiatry and Clinical Neuroscience, 15, 180-186. 
Rapee, R.M. (2002). The development and modification of temperamental risk for anxiety disorders: Prevention of lifetime anxiety? Biological Psychiatry, 52, 947957.

Reisberg, B., Franssen, E., Sclan, S.G., Kluger, A., \& Ferris, S.H. (1989). Stage specific incidence of potentially remediable behavioral symptoms in aging and Alzheimer disease: A study of 120 patients using the BEHAVE-AD. Bulletin of Clinical Neurosciences, 54, 95-112.

Russ, T.C., Hamer, M., Stamatakas, E., Starr, J.M., \& Batty, G.D. (2011). Psychological distress as a risk factor for dementia death. Archives of Internal Medicine, 171, 1858-1859.

Ryu, S.H., Ha., J.H., Park, D.H., Yu, J., \& Livingston, G. (2011). Persistence of neuropsychiatric symptoms over six months in mild cognitive impairment in community-dwelling Korean elderly. Internation Psychogeriatrics, 23, 214-220.

Sagen, V., Wike, T.G., Moum, T., Mørland, T., Finset, A., \& Dammen, T. (2009). Screening for anxiety and depression after stroke: Comparison of the Hospital Anxiety and Depression Scale and the Montgomery and Asberg Depression Rating Scale. Journal of Psychosomatic Research, 67, 325-332.

Saliba, D., \& Buchanan, J. (2008). Development and validation of a revised nursing home assessment tool: MDS 3.0. Retrieved from http://www.cms.hhs.gov/NursingHomeQualityInits/downloads/MDS30FinalRepo $\underline{\text { rt.pdf }}$

Schoevers, R.A., Beekman, A.T.F., Deeg, D.J.H., Jonker, C., \& van Tilburg, W. (2003). Comorbidity and risk-patterns of depression, generalized anxiety disorder and 
mixed anxiety depression in later life: Results from the AMSTEL study. International Journal of Geriatric Psychiatry, 18, 994-1001.

Schoevers, R.A., Deeg, D.J.H., van Tilburg, W., \& Beekman, A.T.F. (2005). Depression and Generalized Anxiety Disorder: Co-occurrence and longitudinal patterns in elderly patients. American Journal of Geriatric Psychiatry, 13, 31-39.

Seblega, B.K., Zhang, N.J., Unruh, L.Y., Breen, G., Paek, S.C., \& Wan, T.T.H. (2010). Changes in nursing home staff levels, 1997 to 2007. Medical Care Research and Review, 67, 232-246.

Segal, D.L., Kabacoff, R.I., Hersen, M., Van Hasselt, V.B., \& Ryan, C.F. (1995). Update on the reliability of diagnosis in older psychiatric outpatients using the Structured Clinical Interview for DSM-III-R. Journal of Clinical Geropsychology, 1, 313321.

Seitz D., Purandare N., \& Conn, D. (2010). Prevalence of psychiatric disorders among older adults in long-term care homes: A systematic review. International Psychogeriatrics, 4, 1-15.

Selbæk, G., \& Engedal, K. (2012). Stability of the factor structure of the neuropsychiatric inventory in a 31-month follow-up study of a large sample of nursing home patients with dementia. International Psychogeriatrics, 24, 62-73.

Shankar, K.K., \& Orrell, M.W. (2000). Detecting and managing depression and anxiety in people with dementia. Current Opinion in Psychiatry, 13, 55-59.

Sheikh, J., Yesavage, J.A., Brooks, J.O., Friedman, L., Gratzinger, P., Hill, R.D. et al. (1991). Proposed factor structure of the Geriatric Depression Scale. International Psychogeriatrics, 3, 23-28. 
Smallbrugge, M., Jongenelis, L., Pot, A.M., Beekman, A.T.F., \& Eefsting, J.A. (2005). Comorbidity of depression and anxiety in nursing home patients. International Journal of Geriatric Psychiatry, 20, 218-226.

Smallbrugge, M., Pot, A.M., Jorgenelis, K., Beekman, A.T.F., \& Eefsting, J.A. (2005). Prevalence and correlates of anxiety among nursing home patients. Journal of Affective Disorders, 88, 145-153.

Snow, A.L., Huddleston, C., Robinson, C., Kunik, M.E., Bush, A.L., Wilson, N.,...Stanley, M.A. (2012). Psychometric properties of a structured interview guide for the rating for anxiety in dementia. Aging \& Mental Health. doi: $10.1080 / 13607863.2011 .644518$

Sourial, R., McCusker, J., Cole, M., \& Abrahamowicz, M. (2008). Agitation in dementia patients in an acute care hospital: Prevalence, disruptiveness, and staff burden. International Psychogeriatrics, 13, 183-197.

Starkstein, S.E., Jorge, R., Mizrahi, R., \& Robinson, R.G. (2006). A diagnostic formulation for anosognosia in Alzheimer's disease. Journal of Neurology, Neurosurgery, and Psychiatry, 77, 719-725.

Starkstein, S.E., Jorge, R., Petracca, G., \& Robinson, R.G. (2007). The construct of Generalized Anxiety Disorder in Alzheimer disease. American Journal of Geriatric Psychiatry, 15, 42-49.

Stevens, J.P. (2002). Applied multivariate statistics for the Social Solutions $\left(4^{\text {th }}\right.$ ed.). Mahwah, NJ: Lawrence Erlbaum Associates, Inc.

Tariot, P.N., Mack, J.L., Patterson, M.B., Edland, S.D., Weiner, M.F., Fillenbaum, G., et al. (1995). The Behavior Rating Scale for Dementia of the Consortium to 
Establish a Registry for Alzheimer's Disease. The American Journal of Psychiatry, 152, 1349-1357.

Teachman, B.A., Siedlecki, K.L., \& Magee, J.C. (2007). Aging and symptoms of anxiety and depression: Structural invariance of the tripartite model. Psychology and Aging, 22, 160-170.

Tennyson, K.A.B. (2003). Evaluation a cognitive conceptualization of anxiety in older nursing home residents with cognitive impairment, DAI, 64, 3544.

Teri, L., Ferretti, E., Gibbons, L.E., Logsdon, R.G., McCurry, S.M., Kukull, W.A., et al. (1999). Anxiety in Alzheimer's disease: Prevalence and comorbidity. Journals of Gerontology. Series A, Biological Sciences and Medical Sciences, 54, M348-352.

Teri, L., Truax, P., Logsdon, R., Uomoto, J., Zarit, S., \& Vitaliano, P.P. (1992). Assessment of behavioral problems in dementia: The Revised Memory and Behavior Problems Checklist. Psychology and Aging, 7, 622-631.

Thomasma, M., Yeaworth, R.C., \& McCabe, B.W. (1990). Moving day: Relocation and anxiety in institutionalized elderly. Journal of Gerontological Nursing, 16, 18-25.

Trachtenberg, R.E., Weiner, M.F., \& Thal, L.J. (2002). Estimating the prevalence of agitation in community-dwelling persons with Alzheimer's disease. Journal of Neuropsychiatry and Clinical Neuroscience, 14, 11-18.

Twelftree, H., \& Qazi, A. (2006). Relationship between anxiety and agitation in dementia. Aging \& Mental Health, 10, 362-367.

U.S. Census Bureau (2010). Annual estimates of the resident population by sex and selected age groups for the United States: April 1, 2000 to July 1, 2009. Retrieved 
from http://www.census.gov/popest/national/asrh/NC-EST2009/NC-EST2009-

$\underline{02 . x l s}$

Vahia, I.V., Meeks, T.W., Thompson, W.K., Depp, C.A., Zisook, S., Allison, M. et al. (2010). Subthreshold depression and successful aging in older women, American Journal of Geriatric Psychiatry, 18, 212-220.

Vink, D., Aartsen, M.J., \& Schoevers, R.A. (2008). Risk factors for anxiety and depression in the elderly: A review. Journal of Affective Disorders, 106, 29-44.

Warner, R.M. (2013). Applied statistics: From bivariate through multivariate techniques ( $2^{\text {nd }}$ ed.). Thousand Oaks, CA: Sage Publications, Inc.

Weiner, M.F., Tractenberg, R., Teri, L., Logsdon, R., Thomas, R.G., Gamst, A., \& Thal, L.J. (2000). Quantifying behavioral disturbance in Alzheimer's disease patients. Journal of Psychiatric Research, 34, 163-167.

Wilson, R.S., Begeny, C.T., Boyle, P.A., Schneider, J.A., \& Bennet, D.A. (2011). Vulnerability to stress, anxiety, and development of dementia in old age. American Journal of Geriatric Psychiatry, 19, 327-334. 
Table 1

Test of Normality

Kolmogorov-Smirnov

Measure

ARS - Positive Affect

ARS - Negative Affect

RAID Total Score (Resident)

Worry Subscale

Apprehension and Vigilance Subscale

Motor Tension Subscale

Autonomic Hypersensitivity Subscale

RAID Total Score (CNA)

Worry Subscale

Apprehension and Vigilance Subscale

Motor Tension Subscale

Autonomic Hypersensitivity Subscale

CMAI Total Score

Physically Aggressive Subscale

Physically Non-aggressive Subscale

Verbally Agitated Subscale

BIMS

PHQ-9

\begin{tabular}{lll}
$D$ & $d f$ & $p$ \\
\hline .09 & 65 & .20 \\
.24 & 65 & .00 \\
.14 & 65 & .00 \\
.18 & 65 & .00 \\
.23 & 65 & .00 \\
.15 & 65 & .00 \\
.34 & 65 & .00 \\
.19 & 65 & .00 \\
.21 & 65 & .00 \\
.34 & 65 & .00 \\
.19 & 65 & .00 \\
.38 & 65 & .00 \\
.18 & 65 & .00 \\
.46 & 65 & .00 \\
.24 & 65 & .00 \\
.22 & 65 & .00 \\
.16 & 65 & .00 \\
.23 & 65 & .00
\end{tabular}


Table 2

Demographic Characteristics of Ineligible Participants

$n=18$

Age

Length of Stay

Race

European American

15

African American

\begin{tabular}{llll}
$M$ & $S D$ & Frequency & Percent \\
\hline 74.41 & 11.01 & & \\
12.56 & 17.35 & &
\end{tabular}

Sex

Female
Male

3

83.3

Marital Status

Never Married

Married

Widowed

Divorced

$\begin{array}{ll}9 & 50 \\ 9 & 50\end{array}$

50

$2 \quad 11.1$

$3 \quad 16.7$

$\begin{array}{ll}7 & 38.9\end{array}$

$6 \quad 33.3$

Entered From

Acute Hospital Stay

17

94.4

Another Nursing Homes

1

5.6 
Table 3

Descriptive Statistics for Ineligible Participants

Measure

ARS - Positive Affect

ARS - Negative Affect

RAID Total Score (Resident)

Absent

Elevated

RAID Total Score (CNA)

Absent

Elevated

CMAI Total Score

Absent

Present

BIMS

Intact/Borderline

Moderate Impairment

Severe Impairment

PHQ-9

None

Mild

Moderate

Moderately Severe

General Mental Health History

No

Yes

History of Mood or Anxiety Disorder

No

Yes

History of Depression

No

Yes

History of Anxiety

No

Yes

\begin{tabular}{llll}
$M$ & $S D$ & Frequency & Percent \\
\hline 15.33 & 6.35 & & \\
8.28 & 2.97 & & \\
7.39 & 7.79 & & 77.8 \\
& & 14 & 22.2
\end{tabular}

$5.33 \quad 6.54$

15

83.3

16.7

$6.17 \quad 6.68$

3

72.2

27.8

$11.61 \quad 3.26$

$\begin{array}{ll}13 & 72.2 \\ 5 & 27.8\end{array}$

$\begin{array}{ll}9 & 50.0\end{array}$

$7 \quad 38.9$

$2 \quad 11.1$

2.33

4.24

$\begin{array}{ll}15 & 83.3 \\ 1 & 5.6 \\ 1 & 5.6 \\ 1 & 5.6 \\ & \\ 12 & 66.7 \\ 6 & 33.3 \\ & \\ 2 & 11.1 \\ 16 & 89.9 \\ & \\ 10 & 55.6 \\ 8 & 44.4 \\ & \\ 5 & 27.8 \\ 13 & 72.2\end{array}$


Table 4

Demographic Characteristics of Eligible Participants

$n=65$

Age

Length of Stay

Race

European American

African American

Sex

Female

Male

Marital Status

Never Married

Married

Widowed

Divorced

Entered From

Acute Hospital Stay

Another Nursing Homes

Community

Psychiatric Hospital

Inpatient Rehabilitation

Other

\begin{tabular}{llll}
$M$ & $S D$ & Frequency & Percent \\
\hline 82.42 & 7.84 & & \\
23.89 & 20.70 & &
\end{tabular}

57

$8 \quad 12.3$

48

17

73.8

26.2

$\begin{array}{ll}6 & 9.2\end{array}$

$11 \quad 16.9$

$43 \quad 66.2$

$\begin{array}{ll}5 & 7.7\end{array}$

56

86.2

2

3

1

2

1
3.1

4.6

1.5

3.1

1.5 
Table 5

Descriptive Statistics for Eligible Participants

Measure

ARS - Positive Affect

ARS - Negative Affect

RAID Total Score (Resident)

\begin{tabular}{ll}
$M$ & $S D$ \\
\hline 15.34 & 5.08 \\
7.82 & 4.05 \\
7.94 & 6.54
\end{tabular}

Frequency Percent

Absent

Elevated

RAID Total Score (CNA)

Absent

Elevated

CMAI Total Score

Absent

Present

BIMS

Intact/Borderline

Moderate Impairment

Severe Impairment

PHQ-9

None
Mild
Moderate

General Mental Health History

No

Yes

6.52

7.15

9.42

10.25

9.18

4.54

2.29

2.99
46

19

52

13

40

25

21

17

27

55

8

2

41

24

12

53

37

28

19

46
70.8

29.2

80.0

20.0

61.5

38.5

32.3

26.2

41.5

84.6

12.3

3.1

63.1

36.9

18.5

81.5

56.9

43.1

History of Anxiety

No

Yes

Nion

Yes

No

$\begin{array}{lll}19 & 29.2 \\ 46 & 70.8\end{array}$

(1)


Table 6

Factor Loadings for the Single Factor Model (Resident)

PHQ-9

ARS Positive Affect

ARS Negative Affect

RAID Autonomic Hypersensitivity

RAID Worry

RAID Motor Tension

RAID Apprehension and Vigilance

CMAI Verbal Agitation

CMAI Physically Aggressive

CMAI Physically Non-aggressive

\begin{tabular}{|c|c|}
\hline & Error \\
\hline .45 & .02 \\
\hline-.36 & .00 \\
\hline .59 & .00 \\
\hline .39 & .01 \\
\hline .61 & .02 \\
\hline .61 & .01 \\
\hline .69 & .01 \\
\hline .34 & .04 \\
\hline .25 & .01 \\
\hline .26 & .03 \\
\hline
\end{tabular}


Table 7

Factor Loadings for the Single Factor Model (CNA)

PHQ-9

ARS Positive Affect

\begin{tabular}{lll} 
& Distress & Error \\
\hline .08 & .02 & \\
-.08 & .00 & \\
.09 & .00 & \\
.62 & .01 \\
.62 & .01 & \\
.58 & .03 \\
.83 & .03 \\
.70 & .01 \\
.10 & .01 \\
.30 & .01
\end{tabular}

ARS Negative Affect

RAID Autonomic Hypersensitivity

RAID Worry

RAID Motor Tension

RAID Apprehension and Vigilance

CMAI Verbal Agitation

CMAI Physically Aggressive

CMAI Physically Non-aggressive 
Table 8

Bivariate Correlations Measures and Their Subscales (Resident)

\begin{tabular}{|c|c|c|c|c|c|c|c|c|c|c|c|c|c|}
\hline Measure & $\begin{array}{l}\text { ARS } \\
\text { PA } \\
\end{array}$ & $\begin{array}{l}\text { ARS } \\
\text { NA }\end{array}$ & $\begin{array}{l}\text { RAID } \\
\text { Tot. }\end{array}$ & $\begin{array}{l}\text { RAID } \\
\text { Worry }\end{array}$ & $\begin{array}{l}\text { RAID } \\
\text { A \& V }\end{array}$ & $\begin{array}{l}\text { RAID } \\
\text { Motor }\end{array}$ & $\begin{array}{l}\text { RAID } \\
\text { Auto. }\end{array}$ & $\begin{array}{l}\text { CMAI } \\
\text { Tot. }\end{array}$ & $\begin{array}{l}\text { CMAI } \\
\text { Phys. }\end{array}$ & $\begin{array}{l}\text { CMAI } \\
\text { Nonagg. }\end{array}$ & $\begin{array}{l}\text { CMAI } \\
\text { Verb. }\end{array}$ & $\begin{array}{l}\text { PHQ } 9 \\
\text { Tot. }\end{array}$ & $\begin{array}{l}\text { PHQ } 9 \\
\text { NA/Cog. }\end{array}$ \\
\hline ARS PA & - & & & & & & & & & & & & \\
\hline ARS NA & -.21 & - & & & & & & & & & & & \\
\hline RAID Tot. & $-.25^{*}$ & $.43^{* *}$ & - & & & & & & & & & & \\
\hline RAID Worry & -.14 & $.37^{* *}$ & $.76^{* *}$ & - & & & & & & & & & \\
\hline RAID A \&V & $-.32^{* *}$ & $.40^{* *}$ & $.68^{* *}$ & $.35^{* *}$ & & & & & & & & & \\
\hline RAID Motor & -.20 & $.33^{* *}$ & $.70^{* *}$ & $.45^{* *}$ & $.38^{* *}$ & 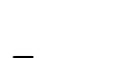 & & & & & & & \\
\hline RAID Auto. & -.02 & $.31^{*}$ & $.53^{* *}$ & $.25^{*}$ & $.44^{* *}$ & .17 & - & & & & & & \\
\hline CMAI Tot. & -.02 & .05 & $.31^{*}$ & $.30^{*}$ & $.25^{*}$ & $.27^{*}$ & -.03 & - & & & & & \\
\hline CMAl Phys. & .06 & .04 & $.24^{*}$ & .16 & $.29^{*}$ & .16 & .11 & $.39^{* *}$ & - & & & & \\
\hline CMAI Nonagg. & -.11 & .16 & .18 & .23 & $.26^{*}$ & .10 & -.03 & $.71^{* *}$ & .15 & & & & \\
\hline CMAI Verb. & -.04 & .10 & $.33^{* *}$ & $.29^{*}$ & .22 & $.30^{*}$ & .02 & $.89^{* *}$ & $.29^{*}$ & $.43^{* *}$ & - & & \\
\hline PHQ 9 Tot. & $-.32^{* *}$ & $.32^{* *}$ & $.25^{*}$ & .02 & $.31^{*}$ & $.29^{*}$ & .03 & .01 & .03 & -.02 & .07 & & \\
\hline $\begin{array}{l}\text { PHQ } 9 \text { NA/Cog. } \\
* p<.05 \text { level. } \\
* * n<01 \text { level }\end{array}$ & -.12 & .13 & .14 & -.04 & .17 & .17 & .08 & -.11 & -.09 & -.14 & -.03 & $.75^{* *}$ & - \\
\hline
\end{tabular}


Table 9

Bivariate Correlations Measures and Their Subscales (CNA)

\begin{tabular}{|c|c|c|c|c|c|c|c|c|c|c|c|c|c|}
\hline Measure & $\begin{array}{l}\text { ARS } \\
\text { PA } \\
\end{array}$ & $\begin{array}{l}\text { ARS } \\
\text { NA }\end{array}$ & $\begin{array}{l}\text { RAID } \\
\text { Tot. }\end{array}$ & $\begin{array}{l}\text { RAID } \\
\text { Worry }\end{array}$ & $\begin{array}{l}\text { RAID } \\
\text { A \& V }\end{array}$ & $\begin{array}{l}\text { RAID } \\
\text { Motor }\end{array}$ & $\begin{array}{l}\text { RAID } \\
\text { Auto. }\end{array}$ & $\begin{array}{l}\text { CMAI } \\
\text { Tot. }\end{array}$ & $\begin{array}{l}\text { CMAI } \\
\text { Phys. }\end{array}$ & $\begin{array}{l}\text { CMAI } \\
\text { Nonagg. }\end{array}$ & $\begin{array}{l}\text { CMAI } \\
\text { Verb. }\end{array}$ & $\begin{array}{l}\text { PHQ } 9 \\
\text { Tot. }\end{array}$ & $\begin{array}{l}\text { PHQ } 9 \\
\text { NA/Cog. }\end{array}$ \\
\hline ARS PA & - & & & & & & & & & & & & \\
\hline ARS NA & -.21 & - & & & & & & & & & & & \\
\hline RAID Tot. & -.21 & $.31^{* *}$ & - & & & & & & & & & & \\
\hline RAID Worry & $-.24^{*}$ & $.31^{* *}$ & $.76^{* *}$ & - & & & & & & & & & \\
\hline RAID A \&V & -.02 & .01 & $.74^{* *}$ & $.46^{* *}$ & - & & & & & & & & \\
\hline RAID Motor & -.14 & .21 & $.76^{* *}$ & $.30^{*}$ & $.47^{* *}$ & - & & & & & & & \\
\hline RAID Auto. & -.07 & -.01 & $.59^{* *}$ & $.39^{* *}$ & $.56^{* *}$ & $.46^{* *}$ & & & & & & & \\
\hline CMAI Tot. & -.02 & .05 & $.57^{* *}$ & $.50^{* *}$ & $.51^{* *}$ & $.39^{* *}$ & .17 & - & & & & & \\
\hline CMAI Phys. & .06 & .04 & .09 & .11 & .07 & .05 & .04 & $.39^{* *}$ & - & & & & \\
\hline CMAI Nonagg. & -.11 & .16 & $.39^{* *}$ & $.33^{* *}$ & .21 & $.35^{* *}$ & -.03 & $.71^{* *}$ & .15 & - & & & \\
\hline CMAI Verb. & -.04 & .10 & $.63^{* *}$ & $.55^{* *}$ & $.58^{* *}$ & $.41^{* *}$ & $.28^{*}$ & $.89^{* *}$ & $.29^{*}$ & $.43^{* *}$ & - & & \\
\hline PHQ 9 Tot. & $-.32^{* *}$ & $.32^{* *}$ & .17 & .22 & .02 & .06 &.- .03 & .01 & .03 & -.02 & .07 & & \\
\hline $\begin{array}{l}\text { PHQ } 9 \text { NA/Cog. } \\
* p<.05 \text { level. }\end{array}$ & -.12 & .13 & -.03 & .04 & -.01 & .06 & .03 & -.11 & -.09 & -.14 & -.03 & $.75^{* *}$ & - \\
\hline
\end{tabular}




\section{Figure 1}

Initial Hierarchical Three Factor Model

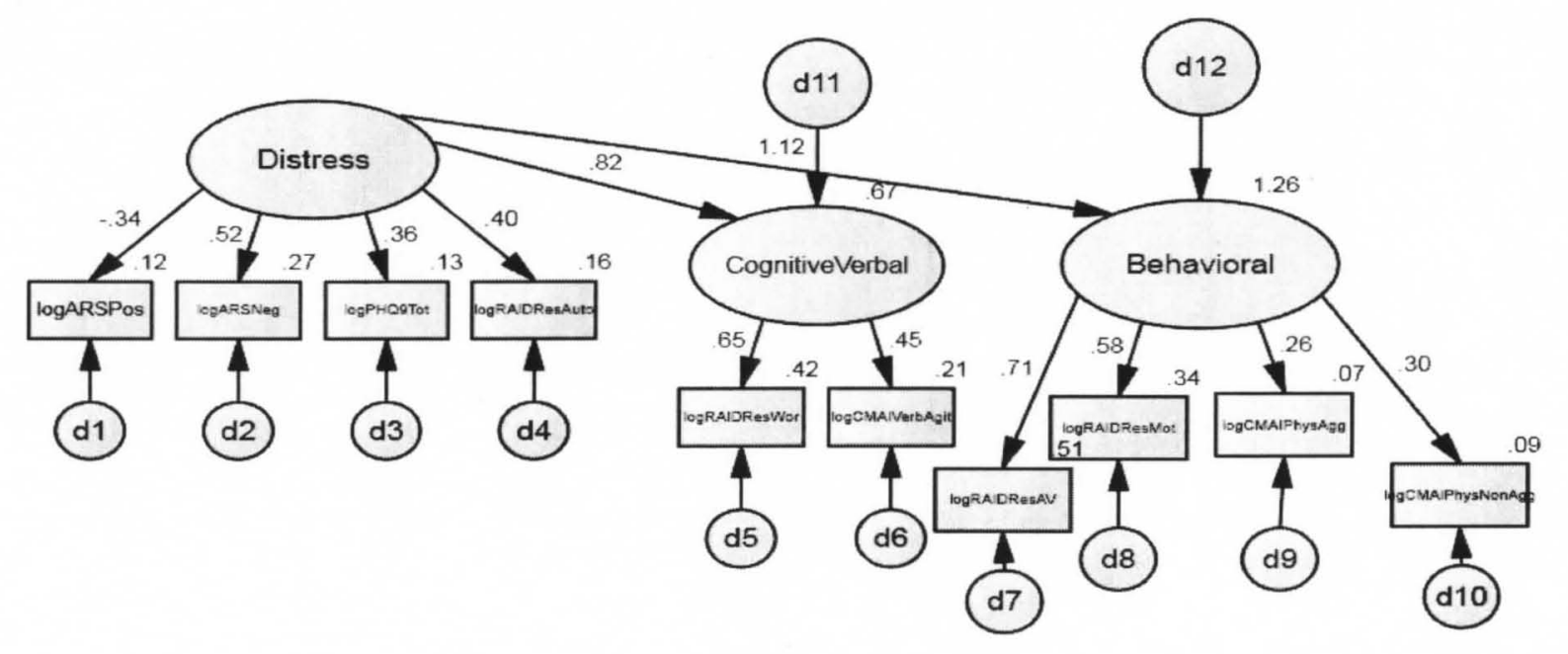


Figure 2

Initial Hierarchical Three Factor Model with Modification Indices

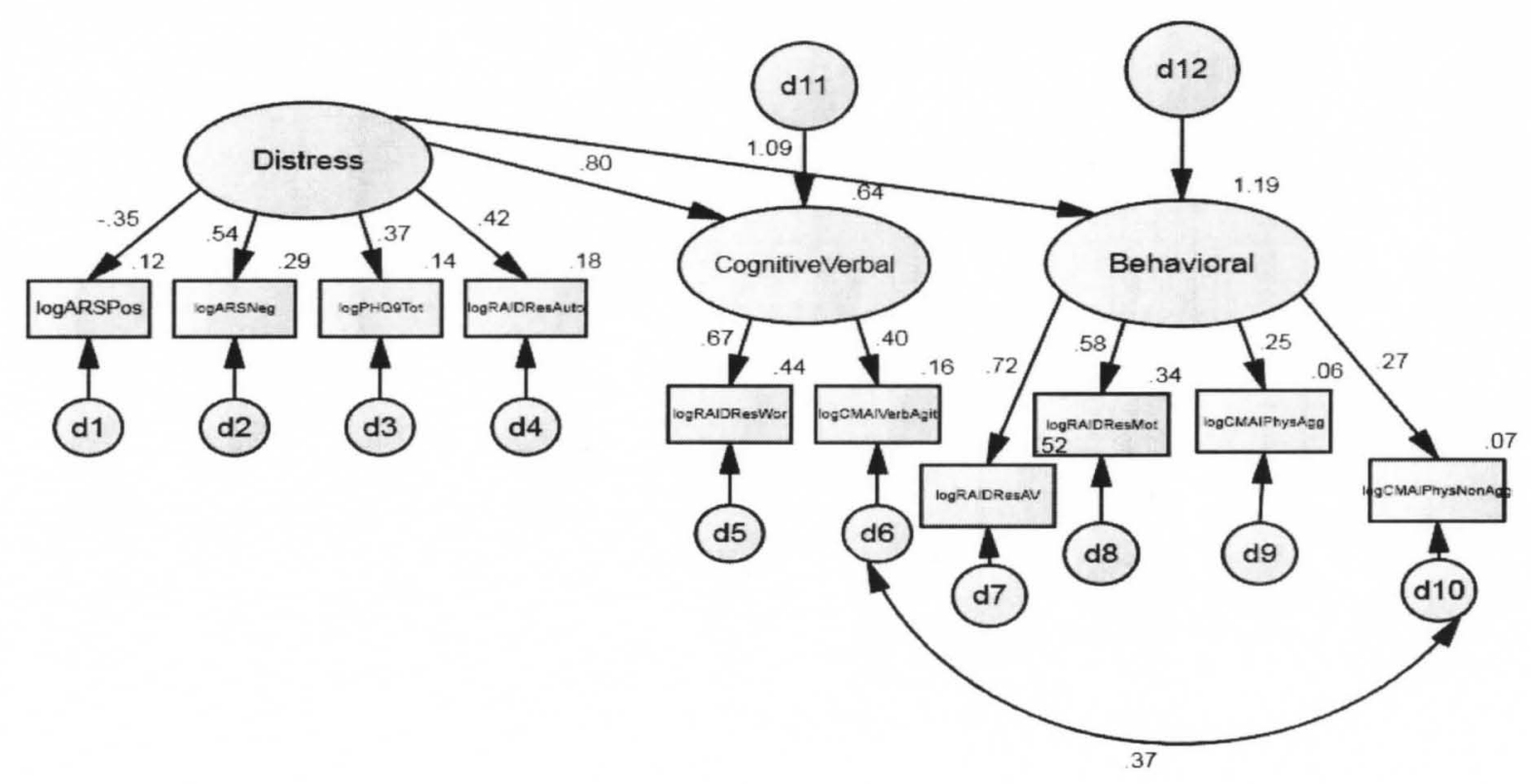




\section{Figure 3}

Revised Single Factor Model (Resident)

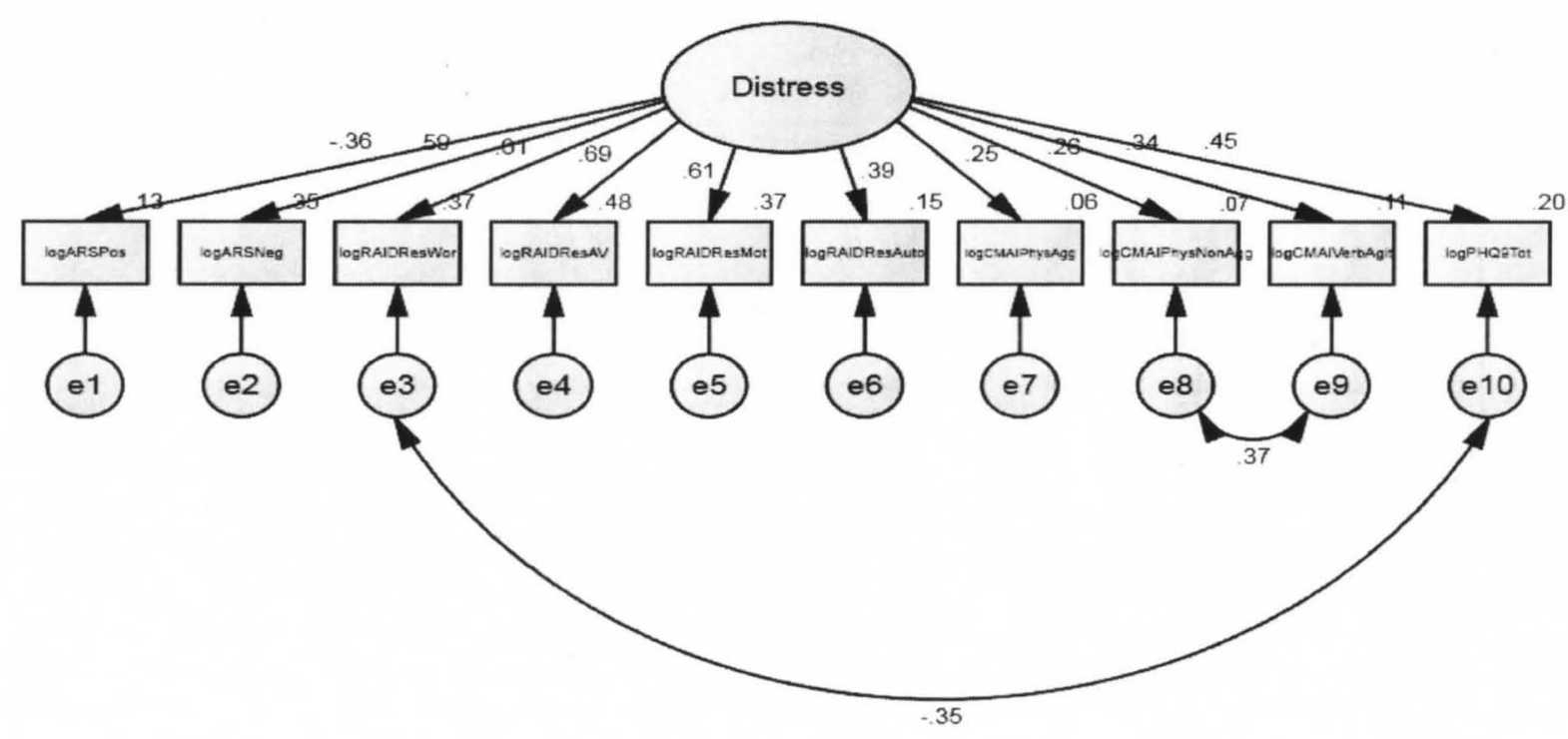


Figure 4

Revised Single Factor Model (CNA)

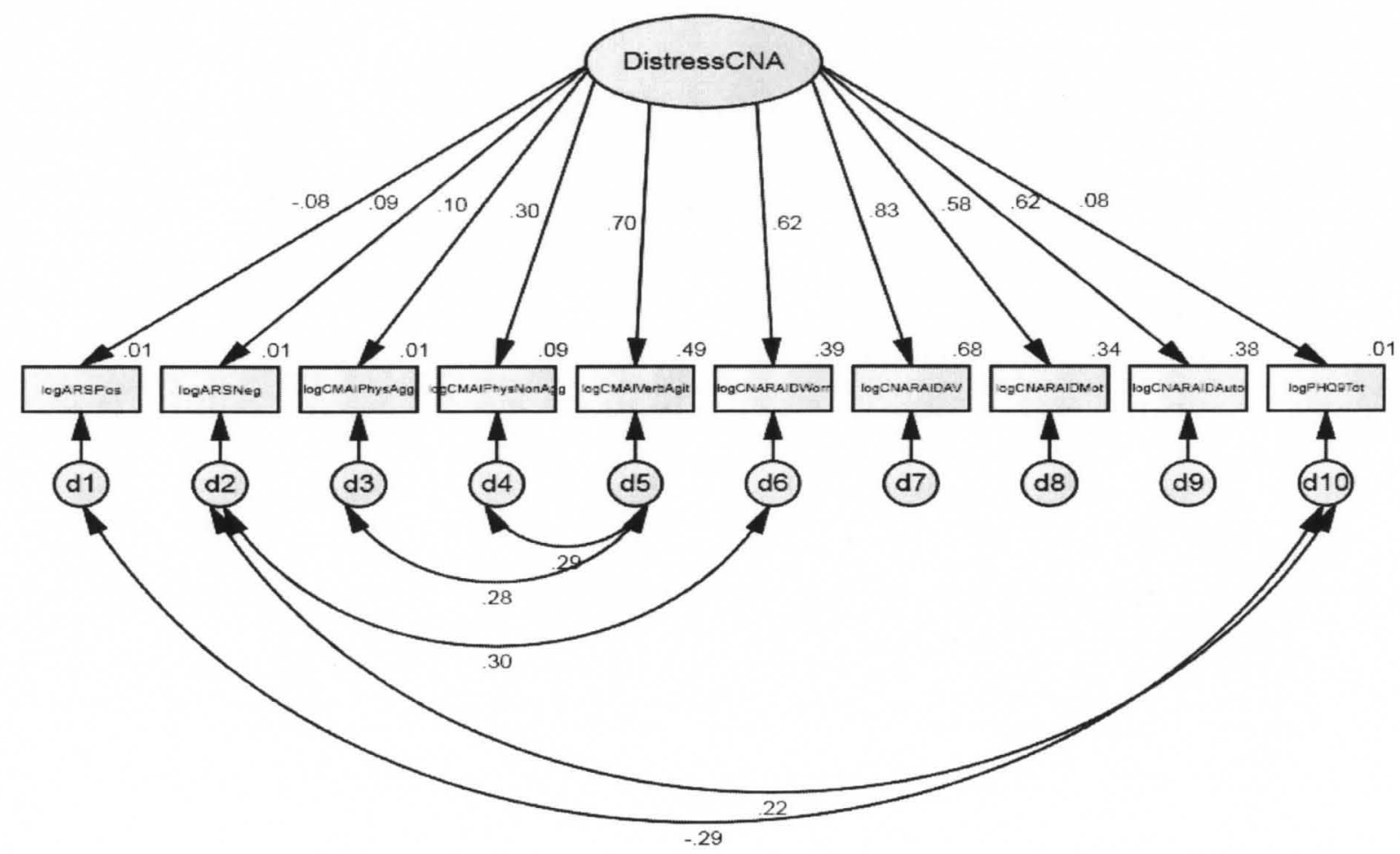




\section{CURRICULUM VITAE}

\section{EDUCATION}

University of Louisville, Louisville, KY $\quad$ PhD 2012

APA Accredited Doctoral Program in Clinical Psychology MA 2008

St. Mary's College of Maryland, St. Mary's City, MD BA 2001

Psychology, Cum Laude

\section{AWARDS}

Graduate Student Research Fund

2008-2011

Arts and Sciences Graduate Student Union

University of Louisville, Louisville, KY

Travel Award, Graduate Student Council

2008-2011

University of Louisville, Louisville, KY

University Fellowship

2006-2008

Department of Psychological and Brain Sciences

University of Louisville, Louisville, KY

St. Mary's Scholar

1999-2001

St. Mary's College of Maryland, St. Mary's City, MD

\section{CLINICAL EXPERIENCE}

Edward Hines, Jr. VA Hospital, Hines, IL

APA Accredited Internship

- Completed rotations in Community Living Center, Blind Rehabilitation Center, Mental Health Clinic, and Primary Care Behavioral Health/Health Promotion Disease Prevention

- Conducted individual and group psychotherapy in long-term care, rehabilitation setting, outpatient clinic, and primary care settings with a socio-economically and ethnically diverse populations

- Delivered Evidence Based Treatments and specialty health psychology and integrated mental health interventions

- Completed intake interviews, inclusive of psychodiagnostic 
screenings, and psychodiagnostic and neuropsychological assessments

- Provided group supervision interns from disciplines other than psychology

- Attended in interdisciplinary team meetings, consulted with treatment team members, provided consultation and feedback to family members

BE-ACTIV: Treating Depression in Nursing Homes

- Conducted individual, 10-week intervention with nursing home residents in metropolitan Louisville on behavioral activation and pleasant event scheduling to alleviate symptoms of depression

- Completed chart reviews, as well as conduct assessments of mood, pleasant events, working alliance, and memory and behavior problems with residents and staff

- Consulted with activities, nursing, and other staff regarding residents' treatment progress and staff members' role in the intervention process

- Acted as consult resource to nursing home during study

Noble H. Kelly Psychological Services Center

Integrative Psychotherapy

Supervisor: Jay Irby, Ph.D.

- Conducted individual psychotherapy with adult client with a focus on the integrative approach to treatment in the university department clinic

Geropsychology Assessment and Intervention Team

Supervisor: Benjamin Mast, Ph.D.

- Completed neuropsychological assessments with communitydwelling older adults in a primary care setting in response to consultations

- Conducted brief neuropsychological assessments with older adults in primary care setting as part of comprehensive multidisciplinary evaluation with immediate feedback to clients, caregivers, and treatment team

Cognitive Behavioral Therapy Team

Supervisor: Janet Woodruff-Borden, Ph.D.

- Conducted individual Cognitive Behavioral Therapy addressing primarily Axis I disorders with adult and child clients in the university department clinic 
- Completed case conceptualizations and school observations, as well as structured interviews for adults and children

Psychological Assessment

$2007-2010$

Supervisors: Paul Bock, Ph.D., Bernadette Walter, Ph.D., David

Winsch, Ph.D.

- Conducted psychological assessments and global cognitive status screenings with child and adult clients, including full diagnostic, Attention-Deficit Hyperactivity Disorder, learning disability, mental retardation, intellectual functioning assessments in the university department clinic

- Conducted intellectual assessments for school-age children for advanced placement in Jefferson County Public School system

Wedgewood Healthcare Center, Clarksville, IN

2007-2008

Supervisor: Suzanne Meeks, PhD.

- Conducted neuropsychological and psychodiagnostic screenings with nursing home residents from Southern Indiana

- Completed training for $B E-A C T I V$ : Treating Depression in Nursing Homes

- Conducted cognitive-behavioral therapy with nursing home resident for treatment of an anxiety disorder

Louisville VA Medical Center Home-Based Primary Care Team, Louisville, KY

Supervisor: Barbara Stetson, Ph.D.

- Conducted in-home screening and psychotherapy with veterans and their family members within the metropolitan Louisville area

- Attended interdisciplinary team meetings and worked with team to provide quality, integrated care

\section{RESEARCH EXPERIENCE}

Dissertation: Anxiety in Older Adults with Dementia Residing in Long-Term Care Facilities

University of Louisville, Louisville, KY

Project Director

Dissertation Committee Chairperson: Suzanne Meeks, Ph.D.

The purpose of this study is to examine the relationship among anxiety, depression, and agitation in older adults with dementia residing in longterm care facilities with an emphasis on the value of global cognitive functioning and mental health history on the experience of anxiety and their ability to differentiate among anxiety and agitation. Long-term care residents in the Louisville metropolitan area complete the Rating Anxiety in Dementia (RAID) scale and mental health history. Data is gathered from their Certified Nursing Assistants about agitated behaviors and 
responsible parties about mental health history, as well as their charts (demographic information, global cognitive functioning, and depression).

Responsibilities include data collection, tracking, and analysis; facility and resident recruitment; and training and oversight of five undergraduates.

Independent Research: Understanding Anxiety in Older Adults with 2008-2012 Dementia Residing in Nursing Homes

University of Louisville, Louisville, KY

Project Director

Supervisor: Suzanne Meeks, Ph.D.

The purpose of this pilot study is to better understand anxiety in older adults with dementia residing in nursing homes in the Louisville metropolitan area and to examine the psychometric properties of RAID scale as used with this population. Responsibilities include recruiting facilities and participants; completing neuropsychological and psychodiagnostic screening with residents and informants; gathering data from charts; and entering, tracking, and analyzing data for presentations.

BE-ACTIV: Treating Depression in Nursing Homes

University of Louisville, Louisville, KY

Graduate Research Assistant

Principal Investigator: Suzanne Meeks, Ph.D.

The BE-ACTIV program is a pleasant events based intervention designed to treat depression in nursing home residents in the Louisville metropolitan area. The purpose of this study is to examine the effectiveness of a 10-week intervention for nursing home residents with depression. Responsibilities include conducting intervention with residents; collecting data from residents, activities staff, and CNAs, including assessments of mood, pleasant events, working alliance, and memory and behavior problems; completing chart reviews on depression diagnoses and medication; and entering data.

Wedgewood Healthcare Center, Clarksville, IN

University Fellow

Supervisor: Suzanne Meeks, Ph.D.

Assisted fellow student with data collection for honors research, including obtaining demographic, health, and behavior information from the residents' Minimum Data Sets and assessments of global cognitive functioning and mood and entering data.

Education and Awareness of Asthma Control

Social Solutions International, Inc., Olney, MD

Project Director

Principal Investigator: Susanna Nemes, Ph.D.

Organized and executed a public awareness campaign to improve asthma management among older adults in Baltimore, MD, including conducting 
a literature review, compiling a database of asthma-related health education materials, developing health promotion materials, providing health promotion messages at events serving older adults, implementing an asthma education and awareness event for older adults at a house of worship, and conducting asthma education courses at senior centers.

Senior Alcohol Abuse: The Hidden Epidemic

Danya International, Inc., Silver Spring, MD

Research Associate

Principal Investigator: Susanna Nemes, Ph.D.

The purpose of this program was to educate participants about the use of alcohol and the implications of excessive or abusive use, and thus motivate them toward changing their drinking behavior. Completed a literature review for course content development, and reviewed and revised content for facilitator's guide and older adult workbook. Worked collaboratively with other staff to complete Phase II proposal submission.

Training Healthcare Providers on Senior Substance Abuse

Danya International, Inc., Silver Spring, MD

Research Associate

Principal Investigator: Susanna Nemes, Ph.D.

The purpose of this study was to train health care providers on how to identify and treat substance abuse in older adults. Conducted the literature review to form the foundation of the online course content, developed demographic forms and feedback survey for the pilot study, recruited participants for the pilot study, and conducted the pilot study of the online course. Worked collaboratively with other staff to complete Phase II proposal submission.

Multi-Problem Youth Screening and Assessment Package Danya International, Inc., Silver Spring, MD

Project Director

The goal of this project was to develop a screening and assessment system to rapidly identify multi-problem youth. Coordinated project activities, staff, and budget; developed project materials content; and supported coordination of expert advisory panel. Conducted a literature review, focus group with youth prevention and intervention professionals, and feasibility test of the screening instrument.

Organize Expert Panel on Primary Prevention of Type 2 Diabetes Danya International, Inc., Silver Spring, MD

Project Coordinator

Convened an expert panel to assess the current state of knowledge available to translate evidence into practice and policy for the primary prevention of diabetes, as well as to identify the major unresolved questions, challenges, and barriers to effective implementation of primary 
prevention efforts.

Formative Research: Decision-making and Risk Perception in Health 2005-2006 Danya International, Inc., Silver Spring, MD

Project Coordinator

Convened an expert panel to assess and integrate up-to-date research on decision-making, motivation, and their influence on risk perception and lifestyle choices, as well as to ascertain how these processes relate to the prevention of Diabetes Mellitus Type 2.

\section{Behavioral Health Screening and Assessment Package}

2003-2004

Danya International, Inc., Silver Spring, MD

Research Associate

Developed and evaluated a user-friendly screening system designed to rapidly identify potential mental health disorders that commonly co-occur with substance-related disorders.

Tobacco Prevention and Control Knowledge Management System 2002-2006 Danya International, Inc., Silver Spring, MD

Research Associate/Project Director

The goal of this contract was to develop a Web-based knowledge management system for tobacco prevention and control. Directed all aspects of the Web site development and design. Acted as the liaison between for subcontractors and carried out the day-to-day tasks of administration and management of the project.

Performance Measurement for Treatment Improvement

2002-2005 Danya International, Inc., Silver Spring, MD

Research Associate

Assisted in the development of a Web-based performance measurement system for opioid treatment programs.

Smoking Cessation for Youth

2001-2004

\section{Danya International, Inc., Silver Spring, MD}

Research Associate

Assisted in the development and evaluation of a curriculum and videobased intervention to help teems quit smoking.

\section{PUBLICATIONS}

Hoffman, J., Nemes, S., Weil, J., Zack, S., Munly, K., \& Hess, L. (2008). Evaluation of the ASCENT Smoking Cessation Program for Adolescents. Journal of Smoking Cessation, 3, 1-7

Hess, L., James, I., Kearley, B., Munly, K., \& Nemes, S. (2007). Alcohol and substance abuse among older adults. In Rin Yoshida (Ed.), Trends in Alcohol Abuse and Alcoholism Research. New York: Nova Science Publishers. 
Garrett, G., Nemes, S., Hoffman, J., Libretto, S., Skinstad, A.H., \& Hess, L. (2005). Promising practices in drug treatment: An overview of methodology. Journal of Teaching in the Addictions, 4, 19-44.

Libretto, S., Nemes, S., Namur, J., Garrett, G., Hess, L., Kaplan, L., \& Landis, R. (2005). Promising practices in drug treatment: Findings from Southeast Asia. Journal of Teaching in the Addictions, 4, 67-88.

Nemes, S., Libretto, S., Garrett, G., Johansson, A., \& Hess, L., (2005). Promising practices in drug treatment: Findings from Latin America. Journal of Teaching in the Addictions, 4, 45-66.

Nemes, S., Johansson, A., Hess, L., Weil, J., \& Pach, A. (2002, December). Patterns and trends of drug abuse in Washington, DC: Epidemiologic trends in drug abuse. Proceedings of the Community Epidemiology Work Group meeting. Rockville, MD: National Institutes of Health.

\section{PRESENTATIONS}

Hess, L.S., Williams, J.C., \& Meeks, S. (2012, November). Mental Health History Predicts Anxiety in Long-term Care Residents with Dementia. Poster presented at the Gerontological Society of America $65^{\text {th }}$ Annual Scientific Meeting, San Diego, CA.

Williams, J.C., Hess, L.S., \& Meeks, S. (2011, August). Anxiety and Agitation in Nursing Home Residents with Dementia. Poster presented at the University of Louisville Summer Research Opportunity Program Research Day, Louisville, KY.

Hess, L.S., Shah, S.N., \& Meeks, S. (2009, November). Assessing Anxiety in Nursing Home Residents with Dementia. Poster presented at the Gerontological Society of America $62^{\text {nd }}$ Annual Scientific Meeting, Atlanta, GA.

Hess, L.S., Kearley, B., Thomas, Z., \& James, I. (2008, November). Substance abuse among older adults: Risk, treatment, and relapse. Symposium presented at the Gerontological Society of America $61^{\text {st }}$ Annual Scientific Meeting, National Harbor, MD.

Hess, L. (2008, July). Assessing anxiety and agitation among nursing home residents with Alzheimer's disease. Poster presented at the International Conference on Alzheimer's Disease, Chicago, IL.

Hess, L. (2007, November). The relationship between anxiety and agitation in older adults with dementia and caregiver burden. Symposium presented at the Gerontological Society of America $60^{\text {th }}$ Annual Scientific Meeting, San Francisco, CA.

Petska, D., Wong, Y.H., Libretto, S., Jones, J., Hoffman, J., Motaparthy, V., \& Hess, L. (2007, June). Developing a knowledge management system for tobacco use prevention and control professionals and advocates. Poster presented at the College on Problems of Drug Dependence Conference, Quebec City, Quebec.

Munly, K., Sexton, J., Hess, L.S., Libretto, S., Wong, Y.H., Jones, J., Nemes, S., \& Moolchan, E. (2006, June). Developing a multi-problem screening system for youth. Poster presented at the College on Problems of Drug Dependence Conference, Scottsdale, AZ. 
Munly, K., Hess, L.S., James, I., Kearley, B., Nemes, S., Pelletier, L., \& Moolchan, E. (2006, June). Substance Abuse and older adults: A review of the literature. Poster presented at the College on Problems of Drug Dependence Conference, Scottsdale, AZ.

Hess, L.S., Libretto, S., Landry, M., \& Nemes, S. (2005, December). Developing and feasibility testing a multi-problem screening system for youth. Poster presented at the American Public Health Association $133^{\text {rd }}$ Annual Meeting and Exposition, Philadelphia, PA.

Libretto, S., Hess, L., Linder, N., \& Hile, M. (2005, December). Developing and pilot testing a co-occurring disorders screening instrument for substance abuse treatment settings. Poster presented at the American Public Health Association $133^{\text {rd }}$ Annual Meeting and Exposition, Philadelphia, PA.

Hess, L., Munly, K., Nemes, S., Pelletier, L., \& Landry, M. (2005, August). Training health care providers on senior substance abuse. Poster presented at the 2005 American Psychological Association Annual Convention, Washington, DC.

Hess, L.S., Libretto, S., Nemes, S., Copeland-Linder, N., \& Landry, M. (2005, August). Developing and feasibility testing a multi-problem screening system for youth. Poster presented at the 2005 American Psychological Association Annual Convention, Washington, DC.

Zack, S.L., Nemes, S., Hoffman, J., Weil, J., Munly, K.A., \& Hess, L. (2005, August). Adolescent Smoking Cessation Escaping Nicotine and Tobacco: Evaluating an intervention. Poster presented at the 2005 American Psychological Association Annual Convention, Washington, DC.

Hess, L.S., Libretto, S., Landry, M., Linder, N., \& Moolchan, E. (2005, June). Developing and pilot feasibility testing a multi-problem screening system for youth. Poster presented at the $67^{\text {th }}$ Annual Scientific Meeting of the College on Problems of Drug Dependence, Orlando, FL.

Munly, K., Hess, L., Nemes, S., Pelletier, L., Landry, M., Moolchan, E. (2005, June). Senior alcohol abuse: Problems for older adults and healthcare providers. Poster presented at the $67^{\text {th }}$ Annual Scientific Meeting of the College on Problems of Drug Dependence, Orlando, FL.

Libretto, S.V., Hess, L., Linder, N., Hile, M., \& Moolchan, E. (2005, June). Development of the behavioral health screening and assessment package. Poster presented at the $67^{\text {th }}$ Annual Scientific Meeting of the College on Problems of Drug Dependence, Orlando, FL.

Zack, S.L., Nemes, S., Hoffman, J., Weil, J., Hess, L., \& Moolchan, E. (2005, June). Adolescent Smoking Cessation Escaping Nicotine and Tobacco (ASCENT): Evaluation of a school-based teen tobacco-cessation program. Poster presented at the $67^{\text {th }}$ Annual Scientific Meeting of the College on Problems of Drug Dependence, Orlando, FL.

Munly, K., Nemes, S., Hess, L., Pelletier, L., \& Johansson, A. (2004, November). Senior alcohol abuse: The hidden epidemic. Poster presented at the $132^{\text {nd }}$ Annual American Public Health Association Meeting, Washington, DC.

Zack, S., Weil, J., Nemes, S., Hoffman, J., Munly, K., \& Hess, L. (2004, November). Curriculum-based tobacco cessation for high school smokers: A controlled trial. 
Poster presented at the $132^{\text {nd }}$ Annual American Public Health Association Meeting, Washington, DC.

Pelletier, L., Hess, L., \& Nemes, S. (2004, November). Diffusing evidence-based innovations in opioid addiction care: $A$ Web-based approach. Poster presented at the $132^{\text {nd }}$ Annual American Public Health Association Meeting, Washington, DC.

Pelletier, L., Hess, L., \& Nemes, S. (2004, July). Disseminating evidence-based action in opioid addiction care via the Internet. Oral presentation at the Translating Research into Practice Conference, Washington, DC.

Hess, L., Zack, S., Hoffman, J.A., Weil, J., \& Levin, H. (2003, December). Teen Quit: An interactive Web site for the promotion of adolescent quit efforts. Poster presented at the National Conference on Tobacco of Health, Boston, MA.

Weil, J., Zack, S., Nemes, S., Hoffman, J.A., Hess, L., \& Libretto, S. (2003, December). SMART: Pilot results of a school based prevention program. Poster presented at the National Conference on Tobacco of Health, Boston, MA.

Zack, S., Hoffman, J.A., Nemes, S., Weil, J., Munly, K., Hess, L., \& Levin, H. (2003, December). ASCENT: Evaluation of a school-based smoking cessation program. Oral presentation conducted at the National Conference on Tobacco of Health, Boston, MA.

Nemes, S., Libretto, S., Hoffman, J., Munly, K., Johansson, A., Hess, L., \& Romero, F. (2003, October). A study of promising drug treatment practices in Latin America, Europe, and Asia. Poster presented at the International Council on Alcohol and Addiction, Toronto, Canada.

Zack, S., Hoffman, J., Nemes, S., Weil, J., Munly, K., Hess, L., Levin, H., \& Moolchan, E. (2003, June). A teen stop-smoking research program evaluation: Six-month follow-up of Adolescent Smoking Cessation Escaping Nicotine and Tobacco (ASCENT). Poster presented at the $65^{\text {th }}$ Annual Scientific Meeting of the College on Problem of Drug Dependence, Bal Harbour, FL.

Munly, K., Nemes, S., Pelletier, L., Johansson, A., \& Hess, L. (2003, June). Senior alcohol abuse: The hidden epidemic. Poster presented at the $65^{\text {th }}$ Annual Scientific Meeting of the College on Problem of Drug Dependence, Bal Harbour, FL.

Cook, L., Hoffman, J., Nemes, S., Weil, J., \& Hess, L. (2003, February). Electronic Prevention Community Organizer (ePrevco.com): An online tool for prevention policy advocacy. Poster presented at the $17^{\text {th }}$ National Conference on Chronic Disease Prevention and Control, St. Louis, MO.

Nemes, S., Beschner, G., Johansson, A., \& Hess, L. (2002, December). Washington, DC trends in drug abuse. Poster presented at the Community Epidemiology Work Group Meeting, Miami, FL.

\section{INVITED TALKS}

Zimmerman, J., \& Hess, L. (2009, December). Psychology and African American Older Adults. PowerPoint lecture presented in PAS 314 Black Psychology. University of Louisville, Louisville, KY. 
Allison, K. E., Flores, P., Gordon, M., Hess, L., Rogers, W., \& Zimmerman, J. (2008, July). Maintaining healthy behavior: How to stick with healthy eating. Presentation at Morgan \& Pottinger PSC, Louisville, KY.

Hess, L., (2007, February). Talking with your doctor. Presentation at the Stephen Foster Senior Living Community, Louisville, KY.

\section{WORK EXPERIENCE}

Social Solutions International, Inc., Olney, MD

Research Coordinator, Gerontology

- Coordinated proposal writing efforts for grants and contracts that focus on the health needs of older adults.

- Acted as Project Director for grants and contracts that serve the health needs of older adults.

Danya International, Inc., Silver Spring, MD

Research Associate II

- Conducted research and collected data for several projects.

- Served as Project Director and Research Associate on several studies for NIH and CDC.

- Developed literature reviews, assisted in research design and analysis and background research for projects, wrote extensively for projects and proposal, and assisted in Web site and proposal development.

\section{Annapolitan Assisted Living Facility, Annapolis, MD}

Activities Director, Dementia Unit

- Created and implemented activities calendar specific to residents with dementia.

- Coordinated volunteers to carry out activities with the assisted living facility. Documents dementia residents' activity attendance. Contributed to and edited facility's monthly newsletter.

Hospice of St. Mary's, Leonardtown, MD

Intern

- Worked with multidisciplinary hospice team to acquire insight into real-life issues related to end-stage illness, personal loss, grief, and the psychosocial, emotional, and spiritual aspects of hospice care.

Hillhaven Assisted Living Nursing and Rehabilitation Center

Activities Assistant

- Lead group and one-to-one activities with nursing home residents.

- Assisted in monthly activity planning. 


\section{PROFESSIONAL SERVICES}

Graduate Student Peer Mentor, University of Louisville

2010-2011

Acted as mentor to incoming first year graduate student.

Campus Representative, American Psychological Association of

2008-2010

Graduate Students Advocacy Coordinating Team

University of Louisville

Mobilized campus efforts to respond to legislative advocacy alerts, disseminated and collected information from students to help APAGS address student needs, recruited APAGS members, and performed other functions as charged by APAGS.

\section{VOLUNTEER EXPERIENCE}

Team Captain, Alzheimer's Association Memory Walk

Louisville, KY

Recruited team members and raised funds for the Alzheimer's

Association - Greater Kentucky and Southern Indiana Chapter. To date, over $\$ 4000$ has been raised for this chapter of the Alzheimer's Association.

Volunteer, Senior Olympics, Louisville, KY

Attended volunteer orientation and assisted in volunteer check-in at events.

Volunteer, Alzheimer's Association - Greater Kentucky and

Southern Indiana Chapter, Louisville, KY

Worked with Director of Public Policy to develop Alzheimer's disease

prevalence and incidence statistics for Kentucky.

Community Services Project Coordinator, Bayside Care Center

2000-2001

\section{Lexington Park, MD}

Worked with and coordinated volunteers within the For Goodness Sake Elderly Project for activity planning and special projects for the care center. Lead 2-6 residents in topic specific sensory stimulation activities once a week for two hours.

\section{PROFESSIONAL MEMBERSHIPS}

American Psychological Association

Gerontological Society of America

Psi Chi, National Honor Society for Psychology 